\title{
Asymptotics for the partition function in two-cut random matrix models
}

\author{
T. Claeys*, T. Grava ${ }^{\dagger}$ and K. D. T-R McLaughlin ${ }^{\ddagger}$
}

February 21, 2018

\begin{abstract}
We obtain large $N$ asymptotics for the random matrix partition function

$$
Z_{N}(V)=\int_{\mathbb{R}^{N}} \prod_{i<j}\left(x_{i}-x_{j}\right)^{2} \prod_{j=1}^{N} e^{-N V\left(x_{j}\right)} d x_{j},
$$

in the case where $V$ is a polynomial such that the random matrix eigenvalues accumulate on two disjoint intervals (the two-cut case). We compute leading and sub-leading terms in the asymptotic expansion for $\log Z_{N}(V)$, up to terms that are small as $N \rightarrow \infty$. Our approach is based on the explicit computation of the first terms in the asymptotic expansion for a quartic symmetric potential $V$. Afterwards, we use deformation theory of the partition function and of the associated equilibrium measure to generalize our results to general two-cut potentials $V$. The asymptotic expansion of $\log Z_{N}(V)$ as $N \rightarrow \infty$ contains terms that depend analytically on the potential $V$ and that have already appeared in the literature. In addition our method allows to compute the $V$-independent terms of the asymptotic expansion of $\log Z_{N}(V)$ which, to the best of our knowledge, had not appeared before in the literature. We use rigorous orthogonal polynomial and Riemann-Hilbert techniques which had so far been successful to compute asymptotics for the partition function only in the one-cut case.
\end{abstract}

\section{Introduction}

Consider the problem of finding asymptotics as $N \rightarrow \infty$ for

$$
Z_{N}(V)=\int_{\mathbb{R}^{N}} \prod_{i<j}\left(x_{i}-x_{j}\right)^{2} \prod_{j=1}^{N} e^{-N V\left(x_{j}\right)} d x_{j}
$$

where $V$ is a polynomial of even degree with positive leading coefficient (so the above integral converges). $Z_{N}(V)$ is the partition function corresponding to the unitary invariant random matrix ensemble on the ensemble of Hermitian $N \times N$ matrices with the probability measure

$$
\frac{1}{\tilde{Z}_{N}(V)} e^{-N \operatorname{Tr} V(M)} d M, \quad d M=\prod_{i<j} d \operatorname{Re} M_{i j} d \operatorname{Im} M_{i j} \prod_{j=1}^{N} d M_{j j},
$$

\footnotetext{
*tom.claeys@uclouvain.be

†grava@sissa.it and tamara.grava@bristol.ac.uk

${ }^{\ddagger}$ mcl@math.arizona.edu
} 
where $\tilde{Z}_{N}(V)$ is a normalizing constant chosen such that we have a probability measure. The induced measure on the (random) eigenvalues of this ensemble of random matrices is explicitly given by

$$
\frac{1}{Z_{N}(V)} \prod_{i<j}\left(x_{i}-x_{j}\right)^{2} \prod_{j=1}^{N} e^{-N V\left(x_{j}\right)} d x_{j}
$$

where $Z_{N}(V)$ is given by 1.1$)$.

The study of the asymptotic behavior of $Z_{N}$ gained notoriety because of an ingenious connection to the combinatorics of graphs embedded in Riemann surfaces [7]. It is now an established fact [14, 7, 25] that there is a simple geometric description of a collection of potentials $V$ for which the large $N$ asymptotic expansion is

$$
-\log Z_{N}(V)+\log Z_{N}\left(V_{0}\right)=N^{2} F_{0}(V)+F_{1}(V)+\sum_{j=2}^{J} F_{j}(V) N^{-2 j}+\mathcal{O}\left(N^{-2 J-2}\right),
$$

in which each term $F_{j}$ depends analytically on the coefficients of the polynomial $V$, and is a generating function for graphs which can be embedded in a Riemann surface of genus $j$ [7, 25].

In the above, the "base potential" $V_{0}$ is quadratic, $V_{0}(x)=x^{2} / 2$, and the interest originally was in the asymptotic behavior for potentials $V$ near this base potential. The partition function can be evaluated exactly for the Gaussian external field $\frac{2 x^{2}}{\sigma}$ in terms of a Selberg integral. Indeed in such a case

$$
Z_{N, \sigma}^{G U E}:=Z_{N}\left(V(x)=2 x^{2} / \sigma\right)
$$

is the partition function of the (re-scaled) Gaussian Unitary Ensemble (GUE), and it is given by

$$
Z_{N, \sigma}^{G U E}=(2 \pi)^{N / 2}\left(\frac{\sigma}{4 N}\right)^{N^{2} / 2} \prod_{n=1}^{N}(n !)
$$

see for example [8]. The large $N$ expansion of $\log Z_{N, \sigma}^{G U E}$ is given by

$$
\begin{aligned}
\log Z_{N, \sigma}^{G U E}=-N^{2}\left(\frac{3}{4}-\frac{1}{2} \log \frac{\sigma}{4}\right)+N \log N & +N(\log 2 \pi-1)+\frac{5}{12} \log N \\
& +\frac{1}{2} \log 2 \pi+\zeta^{\prime}(-1)+\mathcal{O}\left(N^{-1}\right)
\end{aligned}
$$

where $\zeta^{\prime}$ denotes the derivative of the Riemann $\zeta$-function, or equivalently

$$
\log \left[\frac{1}{N !} Z_{N, \sigma}^{G U E}\right]=-N^{2}\left(\frac{3}{4}-\frac{1}{2} \log \frac{\sigma}{4}\right)+N \log 2 \pi-\frac{1}{12} \log N+\zeta^{\prime}(-1)+\mathcal{O}\left(N^{-1}\right) .
$$

Writing $V=V_{0}(x)+\sum_{j=1}^{m} t_{j} x^{j}$, the asymptotic expansion 1.4 was shown to be true in [25] for coefficients $\mathbf{t}=\left(t_{1}, \ldots, t_{m}\right)$ such that $|\mathbf{t}|<T$, and $t_{m} \geq \gamma \sum_{j=1}^{m-1}\left|t_{j}\right|$ for some $T>0$ and $\gamma>0$. But while those were simple conditions on the external field, the asymptotic expansion (1.4) was expected to be true in greater generality.

From the seminal work [7, the collection of potentials $V$ for which the above expansion should hold true is best described in terms of support properties of the equilibrium 
measure, which is the unique probability measure $\mu_{V}$ achieving the minimum (over all probability measures $\mu$ on $\mathbb{R}$ ) of

$$
I_{V}(\mu)=\iint \log |x-y|^{-1} d \mu(x) d \mu(y)+\int V(x) d \mu(x) .
$$

For real analytic potentials $V$ with sufficient growth at $\pm \infty$, the equilibrium measure $\mu_{V}$ is supported on a finite union of bounded disjoint intervals and has a smooth density [20]. We say that $V$ is $k$-cut if $\mu_{V}$ is supported on $k$ disjoint intervals, and that it is regular if certain generic conditions hold which we will specify below.

In [8] it was shown that the partition function has the asymptotic expansion (1.4) under the assumption that $V(x)$ is 1-cut and regular and the deformed external field $V_{\tau}(x)=\left(1-\tau^{-1}\right) x^{2}+V\left(\tau^{-1 / 2} x\right)$ is also one-cut regular for all $\tau \in[1,+\infty)$. The asymptotic expansion (1.4) is expected to hold true in the general case in which only the potential $V(x)$ is 1-cut regular. A by-product of the work in the present paper is a proof of this very general result.

The main result of this paper is the existence of a complete asymptotic expansion for the partition function for any polynomial external field $V$ which is 2-cut and regular.

We note that it is possible to establish an asymptotic expansion for derivatives of $\log Z_{N}(V)$ with respect to parameters of the external potential, since there are a variety of explicit formulae relating such logarithmic derivatives of the partition function to quantities for which the asymptotic behavior is computable via Riemann-Hilbert (RH) techniques [22]. In the seminal work [22] it is shown that in the multi-cut case the asymptotic expansion of recurrence coefficients of orthogonal polynomials is expressed in the large $N$ limit through highly oscillatory $\theta$ functions. This result shows that the asymptotic expansion of the partition function $\log Z_{N}(V)$ in the large $N$ limit contains oscillatory terms. However the main issue is to integrate the expansion in parameter space. In the 1-cut case, the knowledge of an asymptotic expansion for derivatives of $\log Z_{N}(V)$ with respect to parameters of the external potential, together with the complete knowledge of the behavior of the partition function for the reference potential $V_{0}(x)=x^{2} / 2$ allows one to integrate in parameter space and obtain an asymptotic expansion for the partition function itself. However, for the two-cut case, integration from the Gaussian case is fraught with difficulties since one must integrate asymptotic expansions across phase transitions, where the nature of the asymptotic expansion changes see e.g. [8, 9, 13, 15, 18]. The central difficulty is actually finding an explicitly computable two-cut regular case. We are able to do this for a symmetric quartic two-cut regular external field, $V_{0}(x)=x^{4}-4 x^{2}$. Once asymptotics for one particular two-cut external field $V_{0}$ are found, one can write any other polynomial two-cut external field $V_{\vec{t}}, \vec{t}=\left(t_{1}, \ldots, t_{2 d}\right)$, as

$$
V_{\vec{t}}(x)=V_{0}(x)+\sum_{j=1}^{2 d} t_{j} x^{j}, \quad t_{2 d}>0 .
$$

Using a suitable deformation of the form

$$
V_{\vec{t}(\tau)}(x)=V_{0}(x)+\sum_{j=1}^{m} t_{j}(\tau) x^{j}, \quad t_{j}(0)=0, \quad t_{j}(1)=t_{j},
$$


one can obtain an identity for $\frac{d}{d \tau} \log Z_{N}\left(V_{\vec{t}(\tau)}\right)$ in terms of orthogonal polynomials. We then show that the collection of all two-cut regular polynomial external fields is pathwise connected. Once a deformation $V_{\vec{t}(\tau)}(x)$ exists connecting $V_{0}$ to $V_{\vec{t}}(x)$ in such a way that $V_{\vec{t}(\tau)}(x)$ is two-cut regular for all $\tau \in[0,1]$, the next task is to establish a uniform asymptotic expansion as $N \rightarrow \infty$ for logarithmic derivatives of the partition function which is valid for any two-cut regular polynomial external field. We determine such expansion and show that each term of the expansion depends analytically on the potential $V$. By integrating from our new base point through the space of parameters to any other two-cut regular case, we establish the existence of the full partition function expansion for any two-cut regular external field.

Let $\left[a_{1}, a_{2}\right] \cup\left[a_{3}, a_{4}\right], a_{1}<a_{2}<a_{3}<a_{4}$ be the end points of the support of the equilibrium measure $\mu_{V}$ with respect to the two-cut regular potential $V$ and let $\Omega$ be the fraction of eigenvalues in the interval $\left[a_{3}, a_{4}\right]$, namely $\int_{a_{3}}^{a_{4}} d \mu_{V}=\Omega$.

Our main result is the determination of the asymptotic expansion of the partition function for a two-cut regular polynomial potential $V$ :

$$
\begin{aligned}
\log Z_{N}(V) & =\log \left(\frac{N !}{\left\lfloor\frac{N}{2}\right\rfloor !\left\lfloor\frac{N+1}{2}\right\rfloor !} Z_{\left\lfloor\frac{N}{2}\right\rfloor, \sigma^{*}}^{G U E} Z_{\left\lfloor\frac{N+1}{2}\right\rfloor, \sigma^{*}}^{G U E}\right) \\
& -N^{2} F_{0}(V)-F_{1}(V)+\log \theta(N \Omega(V) ; B(V))+\mathcal{O}\left(N^{-1}\right)
\end{aligned}
$$

where $F_{0}=I_{V}\left(\mu_{V}\right)$ defined in (1.8) is the so called planar limit [34, $F_{1}=F_{1}(V)$ is defined as

$$
F_{1}=\frac{1}{24} \log \left(\frac{1}{2^{8}}\left(\frac{|A|}{2 \pi}\right)^{12}|\Delta(a)|^{3} \prod_{j=1}^{4}\left|\widehat{\psi}\left(a_{j}\right)\right|\right), \quad \Delta(a)=\prod_{1 \leq i<j \leq 4}\left(a_{j}-a_{i}\right),
$$

where $a_{1}<a_{2}<a_{3}<a_{4}$ are the endpoints of the support of the equilibrium measure minimizing (1.8), and the constant $A$ is the $\alpha$-period of the holomorphic differential defined on the elliptic Riemann surface $y^{2}=\left(z-a_{1}\right)\left(z-a_{2}\right)\left(z-a_{3}\right)\left(z-a_{4}\right)$ given by

$$
A=2 \int_{a_{2}}^{a_{3}} \frac{1}{\prod_{j=1}^{4} \sqrt{\lambda-a_{j}}} d \lambda=-\frac{4 K(k)}{\sqrt{\left(a_{4}-a_{2}\right)\left(a_{3}-a_{1}\right)}}
$$

with $K(k)$ the complete elliptic integral of the first kind with argument $k>0$ defined by

$$
k^{2}=\frac{\left(a_{3}-a_{2}\right)\left(a_{4}-a_{1}\right)}{\left(a_{3}-a_{1}\right)\left(a_{4}-a_{2}\right)} .
$$

Finally, the values $\widehat{\psi}\left(a_{j}\right)$ in 1.10 are given by

$$
\widehat{\psi}\left(a_{j}\right)=\lim _{x \rightarrow a_{j}, x \in \operatorname{supp} \mu} \frac{\pi \psi(x)}{\sqrt{\left|x-a_{j}\right|}},
$$

where $\psi$ is the density of the equilibrium measure $\mu_{V}$ minimizing (1.8). The quantity $F_{1}=F_{1}(V)$ has been first obtained, up to a constant, by solving the loop equations in [1]. The oscillatory term in (1.9) is expressed by the Jacobi theta function with argument $N \Omega$ and period $B, \operatorname{Im}(B)>0$ defined as

$$
\theta(z ; B)=\sum_{n \in \mathbb{Z}} e^{\pi i n^{2} B+2 \pi i n z}, \quad B=i \frac{K\left(k^{\prime}\right)}{K(k)}, \quad k^{\prime}=\sqrt{1-k^{2}} .
$$


Oscillatory terms first appeared in the asymptotics of orthogonal polynomials related to random matrices in the seminal paper [22]. Later oscillatory terms appeared in the asymptotic expansion of the partition function itself in [10]. Numerical evidence of the oscillatory behaviour of the partition function in the large $N$ limit was first obtained in [35]. The first term on the right hand side of (1.9) does not depend on the potential $V$ and is given by products of GUE partition functions defined in (1.5) with $\sigma^{*}=4 e^{3 / 2}$ and where $\lfloor$.$\rfloor stands for the integer part. The constant \sigma^{*}$ is such that $\lim _{N \rightarrow \infty} N^{-2} \log Z_{N, \sigma^{*}}^{G U E}=0$. We remark that while we have established the existence of a complete expansion to all orders, and while with our techniques we can in principle calculate recursively further terms of the asymptotic expansion $(1.9)$, obtaining explicit formulae beyond the first 3 or 4 terms remains a significant, albeit algebraic, challenge.

Up to the first term, the asymptotic expansion in 1.9 has been computed in the physics literature for general classes of $V$, see e.g. [1, 10, 23], including multi-cut $V$ [26, 16]. In particular in [26], all terms of the asymptotic expansion of the logarithm of the partition function $\log Z_{N}(V)$ have been calculated up to an additive term. However, rigorous asymptotic expansions, without any prior assumptions on their existence, are a very delicate issue and have only been obtained very recently [11, 49]. The manuscripts [11, 49] attack the more general problem of determining the asymptotic behavior for general $\beta$. For any two-cut regular potential $V$ the asymptotic expansion (1.9) clearly shows that the additive term

$$
\log \left(\frac{N !}{\left\lfloor\frac{N}{2}\right\rfloor !\left\lfloor\frac{N+1}{2}\right\rfloor !} Z_{\left\lfloor\frac{N}{2}\right\rfloor, \sigma^{*}}^{G U E} Z_{\left\lfloor\frac{N+1}{2}\right\rfloor, \sigma^{*}}^{G U E}\right)
$$

is independent of $V$ and to the best of our knowledge, such expression has not appeared in the literature before. The representation of the asymptotic expansion in [11] and [49] is exceedingly implicit, making comparisons between those papers and our explicit results difficult. Expanding this constant term as $N \rightarrow \infty$ we obtain

$$
\begin{array}{r}
\log Z_{N}(V)=-N^{2} F_{0}(V)+N \log N+(\log 2 \pi-1) N+\frac{1}{3} \log N+\frac{1}{2} \log 2 \pi+2 \zeta^{\prime}(-1) \\
+\frac{1}{6} \log 2-F_{1}(V)+\log \theta(N \Omega(V) ; B(V))+\mathcal{O}\left(N^{-1}\right)
\end{array}
$$

Remark 1.1 The above asymptotic expansion shows that the term of order $N \log N$ and the term of order $N$ are the same as in the Gaussian case (see (1.6)) while the term of order $\log N$ is different from the one-cut case as well as all the smaller order terms. For a general potential $V$ with low regularity properties the fact that $\log Z_{N}=$ $-N^{2} F_{0}+N \log N+N(\log 2 \pi-1)+\mathcal{O}(\log N)$ is proved in the works [47, 42], see also [12].

\section{Statement of results}

The main contributions of this paper are the following:

(1) we use an approach using orthogonal polynomials and $\mathrm{RH}$ problems to obtain large $N$ asymptotics of the partition function $Z_{N}(V)$ for symmetric quartic polynomials $V$; 
(2) we prove that any one-cut regular polynomial $V(x)$ can be deformed continuously to the Gaussian $x^{2} / 2$ in such a way that the deformed external field remains onecut regular and polynomial of degree $\leq \operatorname{deg} V$ throughout the deformation;

(3) we prove that any two-cut regular polynomial $V$ can be deformed continuously to a symmetric quartic polynomial $V_{0}$ in such a way that the deformed external field remains two-cut supported and polynomial of degree $\leq \operatorname{deg} V$ throughout the deformation;

(4) for any two-cut regular polynomial external field $V_{\vec{t}}(x)=V_{0}(x)+\sum_{j=1}^{2 d} t_{j} x^{j}$, with $t_{2 d}>0$ and $V_{0}$ some quartic symmetric reference potential, we establish an asymptotic expansion for derivatives of $\log Z_{N}\left(V_{\vec{t}}\right)$, with respect to the parameters $t_{j}$. We then obtain the large $N$ asymptotics of the partition function $\log Z_{N}\left(V_{\vec{t}}\right)$ by integration in parameter space from the reference potential $V_{0}$ to $V_{\vec{t}}$.

\subsection{Symmetric quartic $V$}

We first consider the case where $V$ is a symmetric quartic polynomial:

$$
V_{r, s}(z)=\frac{1}{s}\left(x^{4}-r x^{2}\right), \quad r>2 \sqrt{s} .
$$

Then the equilibrium measure minimizing 1.8 is given by [8, 43]

$$
d \mu_{r, s}(x)=\frac{2}{\pi s}|x| \sqrt{\left(b-x^{2}\right)\left(x^{2}-a\right)} d x, \quad x \in[-\sqrt{b},-\sqrt{a}] \cup[\sqrt{a}, \sqrt{b}],
$$

where

$$
a=\frac{1}{2}(r-2 \sqrt{s}), \quad b=\frac{1}{2}(r+2 \sqrt{s}) .
$$

The condition $r>2 \sqrt{s}$ is needed to have a two-cut supported equilibrium measure. In order to derive large $N$ asymptotics for $Z_{N}(r, s):=Z_{N}\left(V_{r, s}\right)$, we will need a number of identities which hold for finite $N$. The following identities will be shown in Section 3.2 and are crucial for our analysis.

Proposition 2.1 Let $V$ be given by (2.1) and $Z_{N}(r, s)$ by (1.1). Then we have

$$
\begin{aligned}
& \log Z_{2 N}(r, s)=\log (2 N) !+\log \widehat{Z}_{N}(-1 / 2 ; r, s)+\log \widehat{Z}_{N}(1 / 2 ; r, s), \\
& \log Z_{2 N+1}(r, s)=\log (2 N+1) !+\log \widehat{Z}_{N+1}\left(-1 / 2 ; r, s_{+}\right)+\log \widehat{Z}_{N}\left(1 / 2 ; r, s_{-}\right),
\end{aligned}
$$

where

$$
s_{ \pm}=s\left(1 \pm \frac{1}{2 N+1}\right)
$$

and

$$
\widehat{Z}_{N}(\alpha ; r, \sigma)=\frac{1}{N !} \int_{\mathbb{R}_{+}^{N}} \prod_{i<j}\left(x_{i}-x_{j}\right)^{2} \prod_{j=1}^{N} x_{j}^{\alpha} e^{-\frac{2 N}{\sigma}\left(x_{j}^{2}-r x_{j}\right)} d x_{j} .
$$


This reduces the problem of finding asymptotics for $Z_{N}(r, s)$ to the problem of finding asymptotics for $\log \widehat{Z}_{N}(\alpha ; r, \sigma)$ as $N \rightarrow \infty$ where $\sigma=s$ and $\sigma=s_{ \pm}=s\left(1 \mp \frac{1}{2 N+1}\right)$. Defining $\frac{d}{d \alpha} \log \widehat{Z}_{N}(\alpha ; r, \sigma)=G_{N}(\alpha ; r, \sigma)$, one has

$$
\log \widehat{Z}_{N}(\alpha ; r, \sigma)=\log \widehat{Z}_{N}(0 ; r, \sigma)+\int_{0}^{\alpha} G_{N}\left(\alpha^{\prime} ; r, \sigma\right) d \alpha^{\prime}
$$

and we will use a second crucial identity, which we will also prove in Section 3.2 , expressing $G_{N}(\alpha ; r, \sigma)$ completely in terms of orthogonal polynomials with respect to the weight $x^{\alpha} e^{-N V_{r, \sigma}(x)}$ on the half-line $[0,+\infty)$, see (3.41) and 3.26$)$ below. Moreover, we will show in Section 3.2 that $\log \widehat{Z}_{N}(0 ; r, \sigma)$ can be expressed for large $N$, up to an exponentially small error, in terms of the partition function for the GUE, namely

$$
\log \widehat{Z}_{N}(0 ; r, \sigma)=\log \left(\frac{e^{\frac{N^{2} r^{2}}{2 \sigma}}}{N !} Z_{N, \sigma}^{G U E}\right)+\mathcal{O}\left(e^{-c N}\right), \quad c>0, \quad N \rightarrow \infty .
$$

We will show in Section 5 using a $\mathrm{RH}$ analysis that $G_{N}(\alpha, r, \sigma)$ admits a full asymptotic expansion in powers of $1 / N$, in which the leading term is proportional to $N$. Combining (2.4)-(2.5) with (2.8), and substituting asymptotics for $G_{N}(\alpha ; r, \sigma)$, we will be able to prove the following result (see Section 5).

Theorem 2.2 Let $Z_{N}=Z_{N}(r, s)$ be the partition function defined by (1.1) with $V=$ $V_{r, s}$ given by (2.1). As $N \rightarrow \infty$ for fixed $s>0, r>2 \sqrt{s}$, there exist constants $c^{(j)}, \widetilde{c}^{(j)}$ for $j \in \mathbb{N}$ such that we have an asymptotic expansion of the form

$$
\begin{aligned}
& \log Z_{N}(r, s)=\log \left[\frac{N ! Z_{\left\lfloor\frac{N}{2}\right\rfloor, \sigma^{*}}^{G U E} Z_{\left\lfloor\frac{N+1}{2}\right\rfloor, \sigma^{*}}^{G U E}}{\left\lfloor\frac{N}{2}\right\rfloor !\left\lfloor\frac{N+1}{2}\right\rfloor !}\right]-N^{2} F_{0} \\
& +\left\{\begin{array}{l}
\frac{1}{2} \log \frac{\sqrt{b}+\sqrt{a}}{2(a b)^{1 / 4}}+\sum_{j=1}^{k} c^{(j)} N^{-j} \quad \text { Neven } \\
\frac{1}{2} \log \frac{\sqrt{b}-\sqrt{a}}{2(a b)^{1 / 4}}+\sum_{j=1}^{k} \widetilde{c}^{(j)} N^{-j} \quad N \text { odd }
\end{array}+\mathcal{O}\left(N^{-k-1}\right),\right.
\end{aligned}
$$

for any $k \in \mathbb{N}$, where $a=\frac{1}{2}(r-2 \sqrt{s})$ and $b=\frac{1}{2}(r+2 \sqrt{s}), F_{0}$ is the limit of the free energy given by

$$
F_{0}(r, s):=-\lim _{N \rightarrow \infty} \frac{1}{N^{2}} \log Z_{N}(r, s)=\frac{3}{8}-\frac{1}{4} \log \frac{s}{4}-\frac{r^{2}}{4 s},
$$

and $Z_{N, \sigma^{*}}^{G U E}$ is defined in (1.5) with $\sigma^{*}=4 e^{3 / 2}$. The coefficients $c^{(k)}(r, s), \tilde{c}^{(k)}(r, s)$ are real analytic functions of $s>0$ and $r>2 \sqrt{s}$, independent of $N$.

Remark 2.3 Using the expansion (1.6), we can write 2.10) in the form

$$
\begin{aligned}
\log Z_{N}=-N^{2} F_{0}+N \log N & +(\log 2 \pi-1) N+\frac{1}{3} \log N+\frac{1}{2} \log 2 \pi+2 \zeta^{\prime}(-1) \\
+ & \frac{1}{6} \log 2+\left\{\begin{array}{ll}
\frac{1}{2} \log \frac{\sqrt{b}+\sqrt{a}}{2(a b)^{1 / 4}} & N \text { even } \\
\frac{1}{2} \log \frac{\sqrt{b}-\sqrt{a}}{2(a b)^{1 / 4}} & \text { Nodd }
\end{array}+\mathcal{O}\left(N^{-1}\right) .\right.
\end{aligned}
$$

Some terms of the above expansions have appeared also in the work [48]. 
Remark 2.4 Our formula 2.10 can be written in the following more general form which is more familiar in the physics literature [1, 26]:

$$
\log \left[\frac{Z_{N}\left\lfloor\frac{N}{2}\right\rfloor !\left\lfloor\frac{N+1}{2}\right\rfloor !}{N ! Z_{\left\lfloor\frac{N}{2}\right\rfloor, \sigma^{*}}^{G U E} Z_{\left\lfloor\frac{N+1}{2}\right\rfloor, \sigma^{*}}^{G U E}}\right]=-N^{2} F_{0}-F_{1}+\log \theta\left(\frac{N}{2} ; B\right)+\mathcal{O}\left(N^{-1}\right)
$$

with $F_{1}$ defined in 1.10 and the theta-function defined in (1.13).

In the symmetric case we consider, by 2.2 , we have

$$
a_{4}=-a_{1}=\sqrt{b}, \quad a_{3}=-a_{2}=\sqrt{a}, \quad \psi(x)=\frac{2}{\pi s}|x| \sqrt{\left(b-x^{2}\right)\left(x^{2}-a\right)},
$$

and

$$
k=\frac{2(a b)^{1 / 4}}{\sqrt{a}+\sqrt{b}}, \quad \Delta(a)=4 \sqrt{a b}(b-a)^{2} .
$$

Using the identity $K(k)=\frac{\pi}{2} \theta(0 ; B)^{2}$, we have

$$
A=-\frac{2 \pi \theta(0 ; B)^{2}}{\sqrt{b}+\sqrt{a}} .
$$

For $N$ even, we can use the periodicity property of the $\theta$-function, $\theta(z+1 ; B)=$ $\theta(z ; B)$, and substitute (2.14), (2.15), and (2.16) into (2.13). Using also the fact that $s=(b-a)^{2} / 4$, we recover (2.10) from 2.13). For $N$ odd, 2.10 again follows from (2.13), but one must also use the identity

$$
\log \theta\left(\frac{1}{2} ; B\right)-\log \theta(0 ; B)=\frac{1}{4} \log \left(1-k^{2}\right)=\frac{1}{2} \log \frac{\sqrt{b}-\sqrt{a}}{\sqrt{b}+\sqrt{a}} .
$$

Remark 2.5 The quantity $F_{1}$ defined in 1.10 coincides with the one defined in 1 , 24, 26] up to a constant factor $\frac{1}{24} \log \left(2^{4} \pi^{-12}\right)$.

Remark 2.6 For values of $r$ near $2 \sqrt{s}$, a phase transition takes place between one-cut and two-cut supported external fields. If $r=2 \sqrt{s}$, the equilibrium density vanishes at an interior point. This situation is studied in detail in [8]: the limit of the free energy $F_{0}(r, s)$ is not analytic near this point, and the subleading term in the large $N$ expansion can be expressed in terms of the Tracy-Widom distribution in a double scaling limit.

\subsection{Equilibrium measures}

We define $P_{m}$ as the space of real polynomials $V$ of even degree $\leq m$ with positive leading coefficient and such that $V(0)=0$, and we write $\mathcal{P}$ for the space of Borel probability measures on $\mathbb{R}$. To $V \in P_{m}$, we associate as before the unique probability measure $\mu_{V} \in \mathcal{P}$ which minimizes the logarithmic energy $I_{V}(\mu)$ defined by 1.8$)$. The equilibrium measure is characterized by the variational conditions [46]

$$
\begin{array}{lr}
2 \int \log |x-y| d \mu_{V}(y)-V(x)=\ell_{V}, & \text { on } \operatorname{supp} \mu_{V}, \\
2 \int \log |x-y| d \mu_{V}(y)-V(x) \leq \ell_{V}, & \text { on } \mathbb{R} .
\end{array}
$$


For a general polynomial $V \in P_{m}, \mu_{V} \in \mathcal{P}$ is supported on a finite union of at most $\operatorname{deg} V / 2$ disjoint bounded intervals. If $V \in P_{m}$ is $k$-cut supported, the measure $\mu_{V}$ has the form

$$
d \mu_{V}(x)=\frac{1}{c} \sqrt{|\mathcal{R}(x)|}|h(x)| d x, \quad x \in \cup_{j=1}^{k}\left[a_{j}, b_{j}\right],
$$

where

$$
\sqrt{R(x)}=\prod_{j=1}^{k}\left(x-a_{j}\right)\left(x-b_{j}\right)
$$

$h$ is a monic polynomial of degree $\operatorname{deg} V-k-1$, and $c$ is a normalizing constant [20]. Generically 2.18) is strict for $x \in \mathbb{R} \backslash \operatorname{supp} \mu_{V}$, but in critical cases, equality can hold at points exterior to the support [41]. If $h(x) \neq 0$ for all $x \in \operatorname{supp} \mu_{V}$ and if (2.18) is strict for all $x \in \mathbb{R} \backslash \operatorname{supp} \mu_{V}$, then $V$ is called $k$-cut regular. We write $P_{m}^{(k)} \subset P_{m}$ for the subset of $P_{m}$ containing all $k$-cut regular external fields of degree $\leq m$.

Example 2.7 A simple example of a one-cut regular external field which we will encounter later on is $V(x)=\frac{2}{s}\left(x^{2}-r x\right)$. The corresponding equilibrium measure is supported on $[a, b]$ with $a$ and $b$ given by 2.3), and is given by

$$
d \mu_{V}(x)=\frac{2}{\pi s} \sqrt{(b-x)(x-a)} d x .
$$

The quartic symmetric polynomial (2.1) is two-cut regular for $r>2 \sqrt{s}$, one-cut regular for $r<2 \sqrt{s}$, and one-cut singular for $r=2 \sqrt{s}$, with an equilibrium density which vanishes in the middle $r / 2$ of its support.

The following result states that any one-cut regular external field $V(x)$ can be deformed continuously to the Gaussian $x^{2} / 2$ in such a way that the deformed external field remains one-cut regular.

Theorem 2.8 Write $V_{\vec{t}}(x)=\frac{x^{2}}{2}+\sum_{j=1}^{m} t_{j} x^{j}$, and let $V=V_{\vec{t}} \in P_{m}^{(1)}, m \geq 2$ be one-cut regular. There exist a continuous path $\vec{t}:[0,1] \rightarrow \mathbb{R}^{m}: \tau \mapsto \vec{t}(\tau)=\left(t_{1}(\tau), \ldots, t_{m}(\tau)\right)$ in $\mathbb{R}^{m}$ such that

(i) $\vec{t}(0)=0$, or equivalently $V_{\vec{t}(0)}(x)=\frac{x^{2}}{2}$,

(ii) $\vec{t}(1)=\vec{t}$

(iii) for all $\tau \in[0,1], V_{\vec{t}(\tau)} \in P_{m}^{(1)}$.

The above theorem will be proved in Section 6 .

Theorem 2.9 Write

$$
V_{\vec{t}}(x)=V_{0}(x)+\sum_{j=1}^{m} t_{j} x^{j}, \quad V_{0}(x)=x^{4}-4 x^{2},
$$

and let $V=V_{\vec{t}} \in P_{m}^{(2)}, m \geq 4$, be two-cut regular. There exist a continuous path $\vec{t}:[0,1] \rightarrow \mathbb{R}^{m}: \tau \mapsto \vec{t}(\tau)=\left(t_{1}(\tau), \ldots, t_{m}(\tau)\right)$ in $\mathbb{R}^{m}$ such that 
(i) $\vec{t}(0)=0$, or equivalently $V_{\vec{t}(0)}(x)=x^{4}-4 x^{2}$,

(ii) $\vec{t}(1)=\vec{t}$

(iii) for all $\tau \in[0,1], V_{\vec{t}(\tau)} \in P_{m}^{(2)}$.

The proof of the above theorem is given in Section 7 .

Remark 2.10 The above results imply that the sets $P_{m}^{(1)}$ and $P_{m}^{(2)}$ are path-connected. Indeed, given two polynomials $V_{1}, V_{2}$ in $P_{m}^{(k)}$ for $k=1,2$, the theorems provide two $k$ cut regular paths which can be composed to obtain a continuous path connecting $V_{1}$ to $V_{2}$. We believe that the set $P_{m}^{(k)}$ is path-connected for any $k \in \mathbb{N}$, but a generalization of our proofs would become considerably more complicated for $k>2$. Theorem 2.8 is not needed for our study of the partition function in the two-cut case, but it allows to extend the proof in [25] of the existence of an asymptotic expansion of the form (1.4) to the entire set of one-cut regular polynomials $V$. This is explained in the remark after Theorem 1.1 in [25].

\subsection{General two-cut regular $V$}

We consider a deformation of the quartic symmetric external field of the form $V_{\vec{t}}(x)=$ $V_{0}(z)+\sum_{j=1}^{2 d} t_{j} x^{j}$, with $t_{2 d}>0, d \geq 2$, and $V_{0}(x)=x^{4}-4 x^{2}$. The deformed external field does not need to be symmetric. Let $P_{n}(x)$ be the orthonormal polynomials with respect to the weight $e^{-N V_{\vec{t}}(x)}$, defined by

$$
\int_{\mathbb{R}} P_{n}(x) P_{m}(x) e^{-N V_{\vec{t}}(x)} d x=\delta_{m n}, \quad P_{n}(x)=\kappa_{n} x^{n}+\ldots,
$$

and let us consider the $2 \times 2$ matrix

$$
X(z)=X^{(N)}(z ; \vec{t})=\left(\begin{array}{cc}
\kappa_{N}^{-1} P_{N}(z) & \frac{1}{2 \pi i} \kappa_{N}^{-1} \int_{\mathbb{R}} P_{N}(s) \frac{e^{-N V_{\vec{t}}(s)} d s}{s-z} \\
-2 \pi i \kappa_{N-1} P_{N-1}(z) & -\kappa_{N-1} \int_{\mathbb{R}} P_{N-1}(s) \frac{e^{-N V_{\vec{t}}^{(s)}} d s}{s-z}
\end{array}\right),
$$

defined for $z \in \mathbb{C} \backslash \mathbb{R}$.

For the partition function 1.1 associated to the external field $V_{\vec{t}}$ we use the relation (see (8.8))

$$
\frac{d}{d t_{k}} \log Z_{N}(\vec{t})=\frac{N}{2} \operatorname{Res}_{z=\infty}\left[\operatorname{Tr}\left(X^{-1}(z ; \vec{t}) X^{\prime}(z ; \vec{t}) \sigma_{3}\right) z^{k}\right]
$$

where ' denotes derivative with respect to $z$. The r.h.s. of the above expression is the derivative with respect of the parameters $t_{k}$ of the isomonodromic $\tau$-function introduced by the Japanese school [33]. The identification of the partition function $Z_{N}(\vec{t})$ with the isomonodromic $\tau$-function was obtained in [5] (see also [6]). For a general potential which can also have singularities, the deformation of the $\tau$-function with respect to parameters in the potential was obtained in [4]. The matrix function $X$ in $(2.23)$ is the solution to the standard $\mathrm{RH}$ problem for the orthogonal polynomials $P_{n}(z)[29$. For a general $j$-cut regular external field $V$, the leading order term in the large $N$ asymptotic expansion for $X$ has been obtained using the Deift/Zhou steepest descent method in [21], and the method used in this paper allows to compute subleading terms as well. 
For our purposes, in the case of a two-cut regular external field $V_{\vec{t}}$ with support $\left[a_{1}, a_{2}\right] \cup\left[a_{3}, a_{4}\right]$ and equilibrium measure $\mu_{V_{\vec{t}}}$, we will need the leading and first subleading term in the asymptotic expansion of the matrix $X(z)$ as $N \rightarrow \infty$. Evaluating the residue in 2.23) and using Fay's identities [28], we obtain the large $N$ asymptotic expansion

$$
\begin{gathered}
-\frac{\partial}{\partial t_{k}} \log Z_{N}\left(V_{\vec{t}}\right)=N^{2} \frac{\partial F_{0}}{\partial t_{k}}-N(\log \theta(N \Omega ; B))^{\prime} \frac{\partial \Omega}{\partial t_{k}}+\frac{\partial F_{1}}{\partial t_{k}}-\frac{\partial}{\partial B} \log \theta(N \Omega ; B) \frac{\partial B}{\partial t_{k}} \\
-(\log \theta(N \Omega ; B))^{\prime \prime} F_{1}^{(1)} \frac{\partial \Omega}{\partial t_{k}}-\frac{F_{0}^{(3)}}{6}\left(\frac{(\theta(N \Omega ; B))^{\prime \prime \prime}}{\theta(N \Omega ; B)}\right)^{\prime} \frac{\partial \Omega}{\partial t_{k}}+\mathcal{O}\left(N^{-1}\right),
\end{gathered}
$$

where $F_{0}=I_{V_{\vec{t}}}\left(\mu_{V_{\vec{t}}}\right)$ is defined in $(1.8)$, the functional form of $F_{1}=F_{1}\left(V_{\vec{t}}\right)$ is defined in 1.10 , and $\theta(N \Omega, B)$ is defined in 11.13 with $\Omega=\int_{a_{3}}^{a_{4}} d \mu_{V_{\vec{t}}}$. The quantities $F_{1}^{(1)}$ and $F_{0}^{(3)}$ are defined in 8.101$)$ and 8.102 respectively and coincide, up to multiplicative factors, with quantities first obtained in [26]. The above expansion has been proved in theorem 8.7. From the above expansion, it is straightforward to arrive to the following theorem.

Theorem 2.11 Let $V_{\vec{t}} \in P_{m}^{(2)}$ be a two-cut regular polynomial external field. The derivative with respect to the parameter $t_{k}, k=1, \ldots, 2 d$ of $\log Z_{N}\left(V_{\vec{t}}\right)$ has the asymptotic expansion

$$
-\frac{\partial}{\partial t_{k}} \log Z_{N}\left(V_{\vec{t}}\right)=\frac{\partial}{\partial t_{k}}\left(N^{2} F_{0}\left(V_{\vec{t}}\right)+F_{1}\left(V_{\vec{t}}\right)-\log \theta\left(N \Omega\left(V_{\vec{t}}\right) ; B\left(V_{\vec{t}}\right)\right)\right)+\mathcal{O}\left(N^{-1}\right),
$$

where $F_{0}=I_{V_{\vec{t}}}\left(\mu_{V_{\vec{t}}}\right)$ is defined in $(1.8)$, the functional form of $F_{1}=F_{1}\left(V_{\vec{t}}\right)$ is defined in (1.10), and $\theta(N \Omega ; B)$ is defined in 1.13) with $\Omega=\int_{a_{3}}^{a_{4}} d \mu_{V_{\vec{t}}}$. The above expansion is uniform for $\vec{t}$ in compact subsets of $\left\{\vec{t} \in \mathbb{R}^{m}: V_{\vec{t}} \in P_{m}^{(2)}\right\}$.

From Theorem 2.9, we can choose a continuous path $\vec{t}:[0,1] \rightarrow \mathbb{R}^{m}: \tau \mapsto \vec{t}(\tau)=$ $\left(t_{1}(\tau), \ldots, t_{m}(\tau)\right)$ in $\mathbb{R}^{m}$ such that $V_{\vec{t}(0)}(x)=x^{4}-4 x^{2}, V_{\vec{t}(1)}(x)=V_{\vec{t}}(x)$, and for all $\tau \in[0,1], V_{\vec{t}(\tau)}$ is two-cut regular. Therefore one can integrate 2.24 to obtain

$$
\begin{aligned}
& \log Z_{N}\left(V_{\vec{t}}\right)-\log Z_{N}\left(V_{0}\right)=-N^{2}\left(F_{0}\left(V_{\vec{t}}\right)-F_{0}\left(V_{0}\right)\right)-F_{1}\left(V_{\vec{t}}\right)+F_{1}\left(V_{0}\right) \\
& \quad+\log \theta\left(N \Omega\left(V_{\vec{t}}\right) ; B\left(V_{\vec{t}}\right)\right)-\log \theta\left(N \Omega\left(V_{0}\right) ; B\left(V_{0}\right)\right)+\mathcal{O}\left(N^{-1}\right), \quad N \rightarrow \infty .
\end{aligned}
$$

Comparing the above relation with 2.10, we obtain our main result.

Theorem 2.12 For a general two-cut regular polynomial potential $V$, the partition function $Z_{N}(V)$ defined in (1.1) has the large $N$ asymptotic expansion

$$
\begin{aligned}
\log Z_{N}(V)=\log & \left(\frac{N !}{\left\lfloor\frac{N}{2}\right\rfloor !\left\lfloor\frac{N+1}{2}\right\rfloor !} Z_{\left\lfloor\frac{N}{2}\right\rfloor, \sigma^{*}}^{G U E} Z_{\left\lfloor\frac{N+1}{2}\right\rfloor, \sigma^{*}}^{G U E}\right) \\
& -N^{2} F_{0}(V)-F_{1}(V)+\log \theta(N \Omega(V) ; B(V))+\mathcal{O}\left(N^{-1}\right)
\end{aligned}
$$

where $F_{0}=I_{V}\left(\mu_{V}\right)$ is defined in (1.8), the functional form of $F_{1}=F_{1}(V)$ is defined in 1.10 , and $\theta(N \Omega, B)=\theta(N \Omega(V)) ; B(V)$ is defined in (1.13) with $\Omega(V)=\int_{a_{3}}^{a_{4}} d \mu_{V}$. The constant $\sigma^{*}$ in the GUE partition function, defined in (1.5), is $\sigma^{*}=4 e^{3 / 2}$. 
The above two theorems are proved in Section 8.7

Remark 2.13 In our derivation of the above formula, we consider the Riemann surface $y^{2}=\left(z-a_{1}\right)\left(z-a_{2}\right)\left(z-a_{3}\right)\left(z-a_{4}\right)$ with the canonical homology basis of cycles $\{\alpha, \beta\}$ as shown in Figure 1. By performing a symplectic transformation

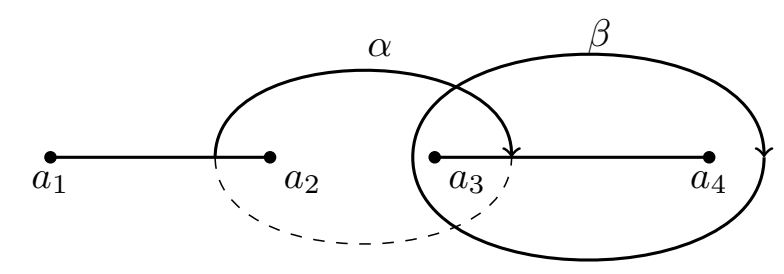

Figure 1: The canonical homology basis.

$$
\tilde{\alpha}=\beta, \quad \tilde{\beta}=-\alpha,
$$

the corresponding modulus $\tilde{B}=-\frac{1}{B}$ and the Jacobi $\theta$-function transform as

$$
\theta(z ; B)=\frac{1}{\sqrt{-i B}} e^{\pi i z^{2} \tilde{B}} \theta(z \tilde{B} ; \tilde{B}) .
$$

Introducing the $\theta$-function with characteristics as

$$
\theta\left[{ }_{\epsilon}^{\delta}\right](z ; B)=\sum_{n \in \mathbb{Z}} e^{\pi i(n+\delta)^{2} B+2 \pi i(z+\epsilon)(\delta+n)},
$$

one verifies by 2.26 that

$$
\theta(N \Omega ; B)=\theta\left[\begin{array}{c}
0 \\
N \Omega
\end{array}\right](0 ; B)=\frac{1}{\sqrt{-i B}} \theta\left[\begin{array}{c}
N \Omega \\
0
\end{array}\right](0 ; \tilde{B}) .
$$

The period $\tilde{A}$ of the holomorphic differential with respect to the basis $\tilde{\alpha}$ takes the form

$$
\tilde{A}=(-i B) A \text {. }
$$

Therefore, combining (2.28) and (2.29), the expansion of the partition function with respect to the homology basis $(\tilde{\alpha}, \beta)$ can be written in the form

$$
\begin{array}{r}
\log Z_{N}\left(V_{\vec{t}}\right)=\log \left(\frac{N !}{\left\lfloor\frac{N}{2}\right\rfloor !\left\lfloor\frac{N+1}{2}\right\rfloor !} Z_{\left\lfloor\frac{N}{2}\right\rfloor, \sigma^{*}}^{G U E} Z_{\left\lfloor\frac{N+1}{2}\right\rfloor, \sigma^{*}}^{G U E}\right) \\
-N^{2} \tilde{F}_{0}-\tilde{F}_{1}+\log \theta\left[\begin{array}{c}
N \Omega \\
0
\end{array}\right](0 ; \tilde{B})+\mathcal{O}\left(N^{-1}\right)
\end{array}
$$

where $\tilde{F}_{0}=F_{0}$ and

$$
\tilde{F}_{1}=\frac{1}{24} \log \left(\frac{1}{2^{8}}\left(\frac{|\tilde{A}|}{2 \pi}\right)^{12}|\Delta(a)|^{3} \prod_{j=1}^{4}\left|\widehat{\psi}\left(a_{j}\right)\right|\right) .
$$

This second choice of homology basis is the one used in [11] and [26]. 


\section{Outline}

The remaining part of the manuscript is organized as follows. In Section 3, we use the orthonormal polynomials with respect to the weight $w_{N}(x ; \alpha, r, \sigma)=x^{\alpha} e^{-\frac{2 N}{\sigma}\left(x^{2}-r x\right)}$ on the half line $[0,+\infty)$ to prove the identities in Proposition 2.1, and we express the partition function $Z_{N}(r, s)$ with respect to the potential $V_{0}(x)=V_{r, s}(x)=\frac{1}{s}\left(x^{4}-r x^{2}\right)$ in terms of quantities related to those polynomials. This will lead to a proof of (2.8) and 2.9$)$.

In Section 4, we obtain leading and subleading asymptotics for the orthogonal polynomials on the half line using a steepest descent analysis of the associated $\mathrm{RH}$ problem.

In Section 5, we use the results from Section 3 and Section 4 to derive the asymptotic expansion of the partition function $\log Z_{N}(r, s)$ as $N \rightarrow \infty$ up to terms of order $\mathcal{O}\left(N^{-1}\right)$, thus proving Theorem 2.2 .

In Section 6, we show that the space of one-cut regular polynomial potential is path-wise connected (Theorem 2.8).

In Section 7, we show that the space of two-cut regular polynomial potential is path-wise connected (Theorem 2.9).

In the last section, we determine the differential identities for the partition function $\log \left(Z_{N}\left(V_{\vec{t}}\right)\right)$ with respect to the parameters $t_{k}$ for a two-cut regular polynomial potential of the form $V_{\vec{t}}(z)=V_{0}(z)+\sum_{k=1}^{2 d} t_{k} z^{k}$ and we calculate the asymptotic expansion as $N \rightarrow \infty$ of these differential identities up to terms of order $\mathcal{O}\left(N^{-1}\right)$. We then integrate in the space of parameters from the reference potential $V_{0}(z)$ up to any two-cut potential $V_{\vec{t}}(z)$, thus proving our main result, Theorem 2.12 .

\section{Orthogonal polynomials and differential identities}

\subsection{Proof of Proposition 2.1}

Let $Z_{N}(V)$ be the partition function defined in (1.1). It is a standard fact that, for general $V$,

$$
Z_{N}(V)=N ! \prod_{n=0}^{N-1} \kappa_{n, N}^{-2}(V),
$$

where $\kappa_{n, N}=\kappa_{n, N}(V)>0$ is the leading coefficient of the degree $n$ orthonormal polynomial defined by

$$
\int_{\mathbb{R}} P_{n, N}(x) P_{m, N}(x) e^{-N V(x)} d x=\delta_{m n}, \quad P_{n, N}(x)=\kappa_{n, N} x^{n}+\ldots
$$

Now let $V=V_{0}=V_{r, s}$ be the quartic symmetric polynomial given by (2.1), and consider orthonormal polynomials $p_{n, N}(x ; \alpha, r, \sigma)$ on $\mathbb{R}^{+}$with respect to the weight

$$
w_{N}(x ; \alpha, r, \sigma)=x^{\alpha} e^{-\frac{2 N}{\sigma}\left(x^{2}-r x\right)},
$$

so that we have the orthogonality conditions

$$
\int_{0}^{+\infty} p_{n, N}(x ; \alpha, r, \sigma) p_{m, N}(x ; \alpha, r, \sigma) w_{N}(x ; \alpha, r, \sigma) d x=\delta_{m n} .
$$


We express the leading and subleading terms as $x \rightarrow \infty$ of $p_{k, N}$ as follows,

$$
p_{k, N}(x ; \alpha, r, \sigma)=\gamma_{k, N}(\alpha, r, \sigma) x^{k}\left(1+\frac{c_{k, N}(\alpha, r, \sigma)}{x}+\frac{d_{k, N}(\alpha, r, \sigma)}{x^{2}}+\ldots\right), \quad \gamma_{k, N}>0 .
$$

Applying the change of variable $x=u^{2}$ to the integral in (3.4) for $\sigma=s$, one obtains orthogonality relations for the polynomials $p_{n, N}\left(u^{2}\right)$ and $u p_{n, N}\left(u^{2}\right)$, and it is straightforward to verify that they obey precisely the orthogonality relations $(3.2)$ with $V=V_{0}=V_{r, s}$ for $P_{2 n, N}$ (with $\alpha=-1 / 2$ ) and $P_{2 n+1, N}$ (with $\alpha=1 / 2$ ), so that by uniqueness we have the identities

$$
P_{2 n, 2 N}(u ; r, s)=p_{n, N}\left(u^{2} ;-1 / 2, r, s\right), \quad P_{2 n+1,2 N}(u ; r, s)=u p_{n, N}\left(u^{2} ; 1 / 2, r, s\right) .
$$

Similar identities have been derived previously, see for example [2, Appendix B], 30], and [17]. In particular, we have by (3.6),

$$
\kappa_{2 k, 2 N}(r, s)=\gamma_{k, N}(-1 / 2 ; r, s), \quad \kappa_{2 k+1,2 N}(r, s)=\gamma_{k, N}(1 / 2 ; r, s) .
$$

Hence, by (3.1), we obtain

$$
\begin{aligned}
& \log Z_{2 N}(r, s)=\log (2 N) !+\log \widehat{Z}_{N}(-1 / 2 ; r, s)+\log \widehat{Z}_{N}(1 / 2 ; r, s), \\
& \log Z_{2 N+1}(r, s)=\log (2 N+1) !+\log \widehat{Z}_{N+1}\left(-1 / 2 ; r, s_{+}\right)+\log \widehat{Z}_{N}\left(1 / 2 ; r, s_{-}\right),
\end{aligned}
$$

with $s_{ \pm}$as in (2.6), and

$$
\log \widehat{Z}_{N}(\alpha ; r, \sigma)=-2 \sum_{n=0}^{N-1} \log \gamma_{n, N}(\alpha ; r, \sigma) .
$$

The quantity $\widehat{Z}_{N}(\alpha ; r, \sigma)$ is the partition function corresponding to a unitary random matrix ensemble for positive-definite matrices, and following standard arguments, it can be expressed as the $N$-fold integral (2.7). This proves Proposition 2.1. In order to obtain asymptotics for $Z_{N}(r, s)$, it is thus sufficient to have asymptotics for $Z_{N}(1 / 2 ; r, \sigma)$ and $\widehat{Z}_{N}(-1 / 2 ; r, \sigma)$ as $N \rightarrow \infty$, with $\sigma=s$ and $\sigma=s_{ \pm}$. The advantage of this formula is that the equilibrium problem associated to the partition function $\widehat{Z}_{N}(\alpha ; r, \sigma)$, is to minimize the quantity

$$
I_{r, \sigma}(\nu)=\iint \log |x-y|^{-1} d \nu(x) d \nu(y)+\frac{2}{\sigma} \int\left(x^{2}-r x\right) d \nu(x)
$$

among all Borel probability measures on $\mathbb{R}^{+}$, and that the support of the minimizer consists of one single interval, as opposed to the support of $\mu_{r, s}$. We have indeed

$$
d \nu_{r, \sigma}(x)=\frac{2}{\pi \sigma} \sqrt{\left(b_{r, \sigma}-x\right)\left(x-a_{r, \sigma}\right)} d x, \quad x \in\left[a_{r, \sigma}, b_{r, \sigma}\right],
$$

which is a semi-circle distribution, with $a_{r, \sigma}>0$ and $b_{r, \sigma}>0$ given by

$$
a_{r, \sigma}=\frac{1}{2}(r-2 \sqrt{\sigma}), \quad b_{r, \sigma}=\frac{1}{2}(r+2 \sqrt{\sigma}),
$$

see Example 2.7. 


\subsection{Differential identity for $\log \widehat{Z}_{N}(\alpha)$}

In what follows, we will consider $r$ and $\sigma$ as fixed parameters, and we omit them in our notations, writing for example $a, b, \nu, \widehat{Z}_{N}(\alpha)$ instead of $a_{r, \sigma}, b_{r, \sigma}, \nu_{r, \sigma}, \widehat{Z}_{N}(\alpha ; r, \sigma)$. In this section, $N$ will also be fixed and we will also use the abbreviated notations $p_{n}, \gamma_{n}, c_{n}, d_{n}, w(x)$ instead of $p_{n, N}, \gamma_{n, N}, c_{n, N}, d_{n, N}, w_{N}(x)$ when there is no possible confusion. We will now derive a differential identity for $\log \widehat{Z}_{N}(\alpha)$ as a function of $\alpha$, following similar ideas as in [39]. By (3.10), we have

$$
\begin{aligned}
\frac{d}{d \alpha} \log \widehat{Z}_{N}(\alpha) & =-2 \sum_{n=0}^{N-1} \frac{\gamma_{n}^{\prime}(\alpha)}{\gamma_{n}(\alpha)} \\
& =-\sum_{n=0}^{N-1} \int_{0}^{+\infty} \frac{\partial}{\partial \alpha}\left(p_{n}^{2}(x ; \alpha)\right) w(x ; \alpha) d x .
\end{aligned}
$$

Recalling the Christoffel-Darboux formula

$$
\sum_{n=0}^{N-1} p_{n}^{2}(x ; \alpha)=a_{N-1}(\alpha)\left(p_{N}^{\prime}(x ; \alpha) p_{N-1}(x ; \alpha)-p_{N}(x ; \alpha) p_{N-1}^{\prime}(x ; \alpha)\right),
$$

where $a_{n}$ is the recurrence coefficient defined by

$$
x p_{n}(x ; \alpha)=a_{n}(\alpha) p_{n+1}(x ; \alpha)+b_{n}(\alpha) p_{n}(x ; \alpha)+a_{n-1}(\alpha) p_{n-1}(x ; \alpha),
$$

and substituting it into (3.14), we obtain

$$
\begin{aligned}
\frac{d}{d \alpha} \log \widehat{Z}_{N}(\alpha)=- & \int_{0}^{+\infty} \frac{\partial}{\partial \alpha}\left(a_{N-1}(\alpha) p_{N}^{\prime}(x ; \alpha) p_{N-1}(x ; \alpha)\right) w(x ; \alpha) d x \\
& +\int_{0}^{+\infty} \frac{\partial}{\partial \alpha}\left(a_{N-1}(\alpha) p_{N}(x ; \alpha) p_{N-1}^{\prime}(x ; \alpha)\right) w(x ; \alpha) d x
\end{aligned}
$$

By the orthogonality conditions for the orthogonal polynomials, we obtain

$$
\begin{gathered}
\frac{d}{d \alpha} \log \widehat{Z}_{N}(\alpha)=-N a_{N-1}^{\prime}(\alpha) \frac{\gamma_{N}(\alpha)}{\gamma_{N-1}(\alpha)}-a_{N-1}(\alpha) \int_{0}^{+\infty}\left(\frac{\partial}{\partial \alpha} p_{N}^{\prime}(x ; \alpha)\right) p_{N-1}(x ; \alpha) w(x ; \alpha) d x \\
-a_{N-1}(\alpha) \int_{0}^{+\infty} p_{N}^{\prime}(x ; \alpha)\left(\frac{\partial}{\partial \alpha} p_{N-1}(x ; \alpha)\right) w(x ; \alpha) d x \\
+a_{N-1}(\alpha) \int_{0}^{+\infty}\left(\frac{\partial}{\partial \alpha} p_{N}(x ; \alpha)\right) p_{N-1}^{\prime}(x ; \alpha) w(x ; \alpha) d x \\
+a_{N-1}(\alpha) \int_{0}^{+\infty} p_{N}(x ; \alpha)\left(\frac{\partial}{\partial \alpha} p_{N-1}^{\prime}(x ; \alpha)\right) w(x ; \alpha) d x
\end{gathered}
$$

The last term in the above equation vanishes because of the orthogonality, and using the orthogonality also for the other integrals we obtain

$$
\frac{d}{d \alpha} \log \widehat{Z}_{N}(\alpha)=-N a_{N-1}^{\prime}(\alpha) \frac{\gamma_{N}(\alpha)}{\gamma_{N-1}(\alpha)}-N a_{N-1}(\alpha) \frac{\gamma_{N}^{\prime}(\alpha)}{\gamma_{N-1}(\alpha)}+a_{N-1}(\alpha)\left(J_{1}-J_{2}\right),
$$

where

$$
\begin{aligned}
& J_{1}=\int_{0}^{+\infty}\left(\frac{\partial}{\partial \alpha} p_{N}(x ; \alpha)\right) p_{N-1}^{\prime}(x ; \alpha) w(x ; \alpha) d x, \\
& J_{2}=\int_{0}^{+\infty} p_{N}^{\prime}(x ; \alpha)\left(\frac{\partial}{\partial \alpha} p_{N-1}(x ; \alpha)\right) w(x ; \alpha) d x .
\end{aligned}
$$


From the recurrence relation 3.16 , it follows directly that $a_{n}=\frac{\gamma_{n}}{\gamma_{n+1}}$, and using this relation we obtain

$$
\frac{d}{d \alpha} \log \widehat{Z}_{N}(\alpha)=-N \frac{\gamma_{N-1}^{\prime}(\alpha)}{\gamma_{N-1}(\alpha)}+\frac{\gamma_{N-1}}{\gamma_{N}}(\alpha)\left(J_{1}-J_{2}\right)
$$

We will simplify this expression further, but for that purpose we need to introduce $w(z ; \alpha)=z^{\alpha} e^{-\frac{2 N}{\sigma}\left(z^{2}-r z\right)}$ as the analytic continuation to $\mathbb{C} \backslash[0,+\infty)$ of the weight function $w(x ; \alpha)$, in such a way that

$$
\lim _{\epsilon \rightarrow 0} w(x+i \epsilon ; \alpha)=w(x ; \alpha), \quad \lim _{\epsilon \rightarrow 0} w(x-i \epsilon ; \alpha)=e^{2 \pi i \alpha} w(x ; \alpha)
$$

for $x \in \mathbb{R}$. In this way we can write integrals on $(0,+\infty)$ as integrals on a contour $\mathcal{C}$ that encircles clockwise the line $(0,+\infty)$ : we have

$$
\int_{0}^{+\infty} P(x) w(x ; \alpha) d x=\frac{1}{1-e^{2 \pi i \alpha}} \int_{\mathcal{C}} P(z) w(z ; \alpha) d z
$$

for any polynomial $P$. The main advantage of the contour $\mathcal{C}$ for us is that it allows for integration of functions with a pole at 0 . We can simplify the formula for $J_{2}$ by integrating by parts and by using the fact that

$$
\frac{\partial}{\partial \alpha} p_{N}(x ; \alpha)=\left(\frac{\partial}{\partial \alpha} p_{N}(x ; \alpha)-\frac{\partial}{\partial \alpha} p_{N}(0 ; \alpha)\right)+\frac{\partial}{\partial \alpha} p_{N}(0 ; \alpha),
$$

and this leads to

$$
\begin{aligned}
J_{2} & =\frac{1}{1-e^{2 \pi i \alpha}} \int_{\mathcal{C}} p_{N}^{\prime}(z ; \alpha)\left(\frac{\partial}{\partial \alpha} p_{N-1}(z ; \alpha)\right) w(z ; \alpha) d z \\
& =-\frac{1}{1-e^{2 \pi i \alpha}} \int_{\mathcal{C}} p_{N}(z ; \alpha)\left(\frac{\partial}{\partial \alpha} p_{N-1}(z ; \alpha)\right)\left(\frac{\alpha}{z}-4 \frac{N}{\sigma} z+2 \frac{N}{\sigma} r\right) w(z ; \alpha) d z \\
& =4 \frac{N}{\sigma} \frac{\gamma_{N-1}^{\prime}(\alpha)}{\gamma_{N}(\alpha)}-\frac{\alpha}{1-e^{2 \pi i \alpha}}\left(\frac{\partial}{\partial \alpha} p_{N-1}(0 ; \alpha)\right) \int_{\mathcal{C}} p_{N}(z ; \alpha) \frac{1}{z} w(z ; \alpha) d z .
\end{aligned}
$$

Similarly

$$
\begin{array}{r}
J_{1}=-N \frac{\gamma_{N}^{\prime}(\alpha)}{\gamma_{N-1}(\alpha)}-\frac{\alpha}{1-e^{2 \pi i \alpha}} \int_{\mathcal{C}}\left(\frac{\partial}{\partial \alpha} p_{N}(z ; \alpha)\right) p_{N-1}(z ; \alpha) \frac{1}{z} w(z ; \alpha) d z \\
-\int_{0}^{+\infty}\left(\frac{\partial}{\partial \alpha} p_{N}(x ; \alpha)\right) p_{N-1}(x ; \alpha)\left(-4 \frac{N}{\sigma} x+2 \frac{N}{\sigma} r\right) w(x ; \alpha) d x .
\end{array}
$$

The second term can be computed as in 3.23 , for the last term we expand $\frac{\partial}{\partial \alpha} p_{N}(x ; \alpha)$ for large $x$ using (3.5) as

$$
\frac{\partial}{\partial \alpha} p_{N}(x ; \alpha)=\gamma_{N}^{\prime}(\alpha) x^{N}+\left(\gamma_{N} c_{N}\right)^{\prime}(\alpha) x^{N-1}+\left(\gamma_{N} d_{N}\right)^{\prime}(\alpha) x^{N-2}+\mathcal{O}\left(x^{N-3}\right) .
$$


This yields

$$
\begin{aligned}
& J_{1}=-(N+\alpha) \frac{\gamma_{N}^{\prime}(\alpha)}{\gamma_{N-1}(\alpha)}-\left(\frac{\partial}{\partial \alpha} p_{N}(0 ; \alpha)\right) \frac{\alpha}{1-e^{2 \pi i \alpha}} \int_{\mathcal{C}} p_{N-1}(z ; \alpha) \frac{1}{z} w(z ; \alpha) d z \\
& +2 \frac{N}{\sigma} \frac{2\left(\gamma_{N} d_{N}\right)^{\prime}(\alpha)-r\left(\gamma_{N} c_{N}\right)^{\prime}(\alpha)}{\gamma_{N-1}(\alpha)}+4 \frac{N}{\sigma} \gamma_{N}^{\prime}(\alpha) \int_{0}^{+\infty} p_{N-1}(x ; \alpha) x^{N+1} w(x ; \alpha) d x \\
& +2 \frac{N}{\sigma}\left(2\left(\gamma_{N} c_{N}\right)^{\prime}(\alpha)-r \gamma_{N}^{\prime}(\alpha)\right) \int_{0}^{+\infty} p_{N-1}(x ; \alpha) x^{N} w(x ; \alpha) d x
\end{aligned}
$$

Note that the above formulas are valid for any $\alpha \in[-1 / 2,1 / 2]$ including $\alpha=0$. The constants $c_{N}$ and $d_{N}$ are defined by (3.5) and determine, together with $\gamma_{N}$, the subleading coefficients of $p_{N}$.

\subsection{Differential identity in terms of $\mathbf{R H}$ solution $Y$}

If we write

$$
Y(z ; \alpha)=Y^{(N)}(z ; \alpha, r, \sigma)=\left(\begin{array}{cc}
\gamma_{N}^{-1} p_{N}(z) & \frac{1}{2 \pi i} \gamma_{N}^{-1} \int_{0}^{+\infty} p_{N}(s) \frac{w(s) d s}{s-z} \\
-2 \pi i \gamma_{N-1} p_{N-1}(z) & -\gamma_{N-1} \int_{0}^{+\infty} p_{N-1}(s) \frac{w(s) d s}{s-z}
\end{array}\right)
$$

$Y$ satisfies the RH problem [29]

\section{RH problem for $Y$}

(a) $Y$ is analytic in $\mathbb{C} \backslash[0,+\infty)$,

(b) $Y$ has boundary values $Y_{ \pm}$for $x \in(0,+\infty)$, and they are related by the jump property

$$
Y_{+}(x)=Y_{-}(x)\left(\begin{array}{cc}
1 & w(x ; \alpha) \\
0 & 1
\end{array}\right), \quad x \in(0,+\infty) .
$$

(c) As $z \rightarrow \infty, Y$ has an asymptotic expansion of the form

$$
Y(z) z^{-n \sigma_{3}}=I+Y_{1} z^{-1}+Y_{2} z^{-2}+Y_{3} z^{-3}+\mathcal{O}\left(z^{-4}\right),
$$

where $Y_{1}, Y_{2}, Y_{3}$ are matrices that may depend on $\alpha, N, r, \sigma$ but not on $z$.

It is straightforward to derive from 3.26 the identities

$$
\begin{array}{ll}
c_{N}=Y_{1,11}, & d_{N}=Y_{2,11} \\
\gamma_{N-1}=\left(\frac{Y_{1,21}}{-2 \pi i}\right)^{1 / 2}, & \gamma_{N}=\left(-\frac{1}{2 \pi i Y_{1,12}}\right)^{1 / 2} \\
\int_{0}^{+\infty} p_{N-1}(x) x^{N} w(x ; \alpha) d x=\frac{Y_{1,22}}{\gamma_{N-1}}, & \int_{0}^{+\infty} p_{N-1}(x) x^{N+1} w(x ; \alpha) d x=\frac{Y_{2,22}}{\gamma_{N-1}} .
\end{array}
$$


We can use those identities to express $J_{1}, J_{2}$, and $\frac{d}{d \alpha} \log \widehat{Z}_{N}(\alpha)$ in terms of $Y=Y^{(N)}$. Indeed, for $J_{2}$, we obtain

$$
J_{2}=2 \frac{N}{\sigma} Y_{1,21}^{\prime} \sqrt{\frac{Y_{1,12}}{Y_{1,21}}}-\left(\frac{Y_{21}(0 ; \alpha)}{-i \sqrt{2 \pi i Y_{1,21}}}\right)^{\prime} \frac{\alpha}{1-e^{2 \pi i \alpha}} \int_{\mathcal{C}} p_{N}(z ; \alpha) \frac{1}{z} w(z ; \alpha) d x
$$

and for $J_{1}$,

$$
\begin{aligned}
& J_{1}=\frac{N+\alpha}{2} \frac{Y_{1,12}^{\prime}}{Y_{1,12}} \frac{1}{\sqrt{Y_{1,12} Y_{1,21}}} \\
& +\frac{2 N}{\sigma \sqrt{Y_{1,12} Y_{1,21}}} \frac{Y_{1,12}^{\prime}}{Y_{1,12}}\left(-\operatorname{Tr} Y_{2}+\frac{r}{2} \operatorname{Tr} Y_{1}-Y_{1,11} Y_{1,22}\right) \\
& +\frac{2 N}{\sigma \sqrt{Y_{1,12} Y_{1,21}}}\left(2 Y_{2,11}^{\prime}-r Y_{1,11}^{\prime}+2 Y_{1,11}^{\prime} Y_{1,22}\right) \\
& \quad-\left(\frac{Y_{11}(0 ; \alpha)}{\sqrt{-2 \pi i Y_{1,12}}}\right)^{\prime} \frac{\alpha}{1-e^{2 \pi i \alpha}} \int_{\mathcal{C}} p_{N-1}(z ; \alpha) \frac{1}{z} w(z ; \alpha) d z
\end{aligned}
$$

In the above formulas, the primes denote derivatives with respect to $\alpha$. The jump condition for $Y$ implies that $\operatorname{det} Y$ is an entire function, and the asymptotics for $Y$ together with Liouvilles theorem yield $\operatorname{det} Y \equiv 1$. In view of (3.28), this can only be true if $\operatorname{Tr} Y_{1}=0$. Combining (3.22), (3.33) and $(3.32)$ one arrives at the formula

$$
\begin{aligned}
& \frac{d}{d \alpha} \log \widehat{Z}_{N}(\alpha)=\frac{\alpha}{2} \frac{Y_{1,12}^{\prime}}{Y_{1,12}}+2 \frac{N}{\sigma}\left(\left(\operatorname{det} Y_{1}\right)^{\prime}+2 Y_{2,11}^{\prime}-r Y_{1,11}^{\prime}\right) \\
& +\frac{N}{2}\left(\frac{Y_{1,12}^{\prime}}{Y_{1,12}}-\frac{Y_{1,21}^{\prime}}{Y_{1,21}}\right) \\
& +\sqrt{Y_{1,12} Y_{1,21}}\left(\frac{Y_{21}(0 ; \alpha)}{-i \sqrt{2 \pi i Y_{1,21}}}\right)^{\prime} \frac{\alpha}{1-e^{2 \pi i \alpha}} \int_{\mathcal{C}} p_{N}(z ; \alpha) \frac{1}{z} w(z ; \alpha) d z \\
& -\sqrt{Y_{1,12} Y_{1,21}}\left(\frac{Y_{11}(0 ; \alpha)}{\sqrt{-2 \pi i Y_{1,12}}}\right)^{\prime} \frac{\alpha}{1-e^{2 \pi i \alpha}} \int_{\mathcal{C}} p_{N-1}(z ; \alpha) \frac{1}{z} w(z ; \alpha) d z .
\end{aligned}
$$

Regarding the last two integrals in the above formulas, we have

$$
Y_{22}(z)=\frac{-2 \pi i \gamma_{N-1}}{1-e^{2 \pi i \alpha}} p_{N-1}(z ; \alpha) w(z ; \alpha)-\frac{\gamma_{N-1}}{1-e^{2 \pi i \alpha}} \int_{\mathcal{C}} \frac{p_{N-1}\left(z^{\prime} ; \alpha\right) w\left(z^{\prime}\right)}{z^{\prime}-z} d z^{\prime},
$$

if $z$ lies inside the contour $\mathcal{C}$. If we set

$$
\tilde{Y}(z)=Y(z)\left(\begin{array}{cc}
1 & -\frac{w(z ; \alpha)}{1-e^{2 \pi i \alpha}} \\
0 & 1
\end{array}\right)
$$

it follows that

$$
\begin{aligned}
& \frac{\alpha}{1-e^{2 \pi i \alpha}} \int_{\mathcal{C}} p_{N-1}\left(z^{\prime} ; \alpha\right) \frac{1}{z^{\prime}} w\left(z^{\prime} ; \alpha\right) d z^{\prime} \\
& \quad=-\frac{\alpha}{\gamma_{N-1}} \lim _{z \rightarrow 0}\left(Y_{22}(z ; \alpha)-\frac{Y_{21}(z ; \alpha) w(z ; \alpha)}{1-e^{2 \pi i \alpha}}\right)=-\frac{\alpha \sqrt{2 \pi}}{\sqrt{i Y_{1,21}}} \tilde{Y}_{22}(0 ; \alpha) .
\end{aligned}
$$


Now, for the other integral in (3.34), the relevant identity is

$$
\gamma_{N} Y_{12}(z)=\frac{1}{2 \pi i\left(1-e^{2 \pi i \alpha}\right)} \int_{\mathcal{C}} \frac{p_{N}\left(z^{\prime} ; \alpha\right) w\left(z^{\prime} ; \alpha\right)}{z^{\prime}-z} d z^{\prime}+\frac{1}{1-e^{2 \pi i \alpha}} p_{N}(z ; \alpha) w(z ; \alpha),
$$

from which it follows that

$$
\begin{array}{r}
\frac{\alpha}{1-e^{2 \pi i \alpha}} \int_{\mathcal{C}} \frac{p_{N}\left(z^{\prime} ; \alpha\right) w\left(z^{\prime} ; \alpha\right)}{z^{\prime}} d z^{\prime}=2 \pi i \alpha \gamma_{N} \lim _{z \rightarrow 0}\left(Y_{12}^{(N)}(z ; \alpha)-\frac{Y_{11}^{(N)}(z ; \alpha) w(z ; \alpha)}{1-e^{2 \pi i \alpha}}\right) \\
=\frac{\sqrt{2 \pi} i \alpha}{\sqrt{-i Y_{1,12}}} \tilde{Y}_{12}(0 ; \alpha) .
\end{array}
$$

Substituting those identities in (3.34), we obtain

$$
\frac{d}{d \alpha} \log \widehat{Z}_{N}(\alpha)=\mathcal{G}_{N}(\alpha)
$$

with

$$
\begin{aligned}
\mathcal{G}_{N}(\alpha)=\mathcal{G}_{N}(\alpha ; r, \sigma)=\frac{\alpha}{2} & \frac{Y_{1,12}^{\prime}}{Y_{1,12}}+2 \frac{N}{\sigma}\left(\left(\operatorname{det} Y_{1}\right)^{\prime}+2 Y_{2,11}^{\prime}-r Y_{1,11}^{\prime}\right) \\
+\frac{N}{2}\left(\frac{Y_{1,12}^{\prime}}{Y_{1,12}}-\frac{Y_{1,21}^{\prime}}{Y_{1,21}}\right)+ & \sqrt{Y_{1,12} Y_{1,21}}\left(\frac{Y_{11}(0 ; \alpha)}{\sqrt{-i Y_{1,12}}}\right)^{\prime} \frac{\alpha}{\sqrt{i Y_{1,21}}} \tilde{Y}_{22}(0 ; \alpha) \\
& -\sqrt{Y_{1,12} Y_{1,21}}\left(\frac{Y_{21}(0 ; \alpha)}{\sqrt{i Y_{1,21}}}\right)^{\prime} \frac{\alpha}{\sqrt{-i Y_{1,12}}} \tilde{Y}_{12}(0 ; \alpha)
\end{aligned}
$$

The identity (2.8) stated in the introduction follows from 3.40). It then follows from (3.8)- (3.9) that

$$
\begin{aligned}
\log Z_{2 N}(r, s)=\log (2 N) !+2 \log \widehat{Z}_{N}(0 ; r, s) & \\
& +\int_{0}^{-1 / 2} \mathcal{G}_{N}(\alpha ; r, s) d \alpha+\int_{0}^{1 / 2} \mathcal{G}_{N}(\alpha ; r, s) d \alpha \\
\log Z_{2 N+1}(r, s)= & \log (2 N+1) !+\log \widehat{Z}_{N+1}\left(0 ; r, s_{+}\right)+\log \widehat{Z}_{N}\left(0 ; r, s_{-}\right) \\
& +\int_{0}^{-1 / 2} \mathcal{G}_{N+1}\left(\alpha ; r, s_{+}\right) d \alpha+\int_{0}^{1 / 2} \mathcal{G}_{N}\left(\alpha ; r, s_{-}\right) d \alpha .
\end{aligned}
$$

The equilibrium measure $\nu_{r, \sigma}$ is one-cut, and we can apply the Deift-Zhou steepest descent method on the RH problem for $Y$ to obtain large $N$ asymptotics for $Y, Y_{1}, Y_{2}$ and derivatives, uniformly for $\alpha \in[-1 / 2,1 / 2]$. This means that we can, in principle, obtain asymptotics for each of the integrals in the above formulas. The only remaining problem is then to find asymptotics for $\widehat{Z}_{N}(0 ; r, \sigma)$. 


\subsection{Asymptotics for $\widehat{Z}_{N}(0 ; r, \sigma)$}

By a shift of the integration variable, we have

$$
\begin{aligned}
\widehat{Z}_{N}(0 ; r, \sigma) & =\frac{1}{N !} \int_{\mathbb{R}_{+}^{N}} \prod_{i<j}\left(x_{i}-x_{j}\right)^{2} \prod_{j=1}^{N} e^{-\frac{2 N}{\sigma}\left(x_{j}^{2}-r x_{j}\right)} d x_{j} \\
& =\frac{e^{\frac{N^{2} r^{2}}{2 \sigma}}}{N !} \int_{\mathbb{R}_{+}^{N}} \prod_{i<j}\left(x_{i}-x_{j}\right)^{2} \prod_{j=1}^{N} e^{-2 \frac{N}{\sigma}\left(x_{j}-\frac{r}{2}\right)^{2}} d x_{j} \\
& =\frac{e^{\frac{N^{2} r^{2}}{2 \sigma}}}{N !} \int_{\left(-\frac{r}{2},+\infty\right)^{N}} \prod_{i<j}\left(x_{i}-x_{j}\right)^{2} \prod_{j=1}^{N} e^{-2 \frac{N}{\sigma} x_{j}^{2}} d x_{j},
\end{aligned}
$$

which is, up to a pre-facor, the partition function for a Gaussian Unitary Ensemble (GUE) with a cut-off at $-r / 2$. We will show that

$$
Z_{N, \sigma}^{(r)}:=\int_{\left(-\frac{r}{2},+\infty\right)^{N}} \prod_{i<j}\left(x_{i}-x_{j}\right)^{2} \prod_{j=1}^{N} e^{-\frac{2 N}{\sigma} x_{j}^{2}} d x_{j}, \quad \sigma<\frac{r^{2}}{4}
$$

is, as $N \rightarrow \infty$, exponentially close to the GUE partition function without a cut-off, given by

$$
Z_{N, \sigma}^{G U E}:=Z_{N, \sigma}^{(-\infty)}=\int_{\mathbb{R}^{N}} \prod_{i<j}\left(x_{i}-x_{j}\right)^{2} \prod_{j=1}^{N} e^{-2 \frac{N}{\sigma} x_{j}^{2}} d x_{j} .
$$

In the GUE (without cut-off) with joint probability distribution of eigenvalues given by

$$
\frac{1}{Z_{N, \sigma}^{G U E}} \prod_{i<j}\left(x_{i}-x_{j}\right) \prod_{j=1}^{N} e^{-\frac{2 N}{\sigma} x_{j}^{2}} d x_{j}
$$

the ratio $Z_{N, \sigma}^{(r)} / Z_{N, \sigma}^{G U E}$ is equal to the probability that all eigenvalues are bigger than $-r / 2$. It is well-known that this probability is exponentially close to 1 since $-r / 2$ is strictly smaller than the left endpoint of the support of the equilibrium measure (which is the limiting mean eigenvalue distribution) corresponding to this ensemble. Indeed, it follows for example by a large deviation principle, see [3, Section 2.6.2], that

$$
\frac{Z_{N, \sigma}^{(r)}}{Z_{N, \sigma}^{G U E}}=1+\mathcal{O}\left(e^{-c N}\right), \quad N \rightarrow \infty, c>0 .
$$

We can conclude that, as $N \rightarrow \infty$,

$$
\begin{aligned}
& \log Z_{2 N}(r, s)=\log (2 N) !+2 \log \left[\frac{1}{N !} Z_{N, s}^{G U E}\right] \\
& +\frac{N^{2} r^{2}}{s}+\int_{0}^{-1 / 2} \mathcal{G}_{N,}(\alpha ; r, s) d \alpha+\int_{0}^{1 / 2} \mathcal{G}_{N}(\alpha ; r, s) d \alpha+\mathcal{O}\left(e^{-c N}\right), \\
& \log Z_{2 N+1}(r, s)=\log (2 N+1) !+\log \left[\frac{1}{(N+1) !} Z_{N+1, s_{+}}^{G U E}\right]+\log \left[\frac{1}{N !} Z_{N, s_{-}}^{G U E}\right] \\
& +\frac{(2 N+1)^{2} r^{2}}{4 s}+\int_{0}^{-1 / 2} \mathcal{G}_{N+1}\left(\alpha ; r, s_{+}\right) d \alpha \\
& +\int_{0}^{1 / 2} \mathcal{G}_{N}\left(\alpha ; r, s_{-}\right) d \alpha+\mathcal{O}\left(e^{-c N}\right)
\end{aligned}
$$


where we used the identity

$$
\frac{(N+1)^{2} r^{2}}{2 s_{+}}+\frac{N^{2} r^{2}}{2 s_{-}}=\frac{(2 N+1)^{2} r^{2}}{4 s} .
$$

\section{Analysis of the RH problem for the orthogonal polyno- mials}

In this section, we will obtain asymptotics for the $\mathrm{RH}$ problem for $Y$, stated in the previous section, as $N \rightarrow \infty$, and this will also lead us towards asymptotics for $\mathcal{G}_{N}$. We recall that $Y$ depends on $N, \alpha, r$, and $\sigma$. By (3.49)-3.50), we will need asymptotics as $N \rightarrow \infty$ for $-\frac{1}{2} \leq \alpha \leq \frac{1}{2}$, and with $\sigma=s$ and $\sigma=s_{ \pm}$. In the case where $\sigma=s_{ \pm}, \sigma$ will be $N$-dependent by (2.6), but this will not cause any problems because the asymptotics for $Y$ will be uniform in $\sigma$ as long as $\sigma<\frac{r^{2}}{4}-\epsilon, \epsilon>0$. Since we assume that $s<\frac{r^{2}}{4}$, we also have that $s_{ \pm}<\frac{r^{2}}{4}-\epsilon$ for $N$ sufficiently large and $\epsilon$ sufficiently small.

\subsection{The equilibrium measure}

Define the equilibrium measure $\nu_{r, \sigma}$ minimizing the logarithmic energy (3.11) among all probability measures on $\mathbb{R}^{+}$. As mentioned in Example 2.7, one can compute $\nu_{r, \sigma}$ explicitly: it is supported on the interval $[a, b]=\left[\frac{r}{2}-\sqrt{\sigma}, \frac{r}{2}+\sqrt{\sigma}\right]$ and the density is given by 3.12 . For all $\sigma<\frac{r^{2}}{4}$, we have that $a>0$. Now, define

$$
g(z)=\int_{a}^{b} \log (z-s) d \nu_{r, \sigma}(s)
$$

where we choose the branch of the logarithm such that $g$ is analytic in $\mathbb{C} \backslash(-\infty, b]$. It is directly verified that

$$
g_{+}(x)-g_{-}(x)=2 \pi i \int_{x}^{b} d \nu_{r, \sigma}(y), \quad \text { for } x<b,
$$

where $\int_{x}^{b} d \nu_{r, \sigma}(y)$ is understood as 1 for $x<a$. For $x \in[a, b]$, the Euler-Lagrange variational conditions conditions for $\nu_{r, \sigma}$ imply that

$$
g_{+}(x)+g_{-}(x)-W(x)-\ell=0, \quad W(x)=\frac{2}{\sigma}\left(x^{2}-r x\right),
$$

for some number $\ell$ depending on $r, \sigma$, and that the left hand side of (4.3) is strictly negative on $(0,+\infty) \backslash[a, b]$.

For later use, we compute the asymptotics for the $g$-function as $z \rightarrow \infty$, which are given by

$$
\begin{aligned}
g(z) & =\log z-\frac{1}{z} \int y d \nu_{r, \sigma}(y)-\frac{1}{2 z^{2}} \int y^{2} d \nu_{r, \sigma}(y)+\mathcal{O}\left(z^{-3}\right) \\
& =\log z-\frac{m_{1}}{z}-\frac{m_{2}}{2 z^{2}}+\mathcal{O}\left(z^{-3}\right)
\end{aligned}
$$

where we write $m_{k}=\int y^{k} d \nu_{r, \sigma}(y)$. It follows that

$$
e^{N g(z) \sigma_{3}}=z^{N \sigma_{3}}\left(I+\frac{G_{1}}{z}+\frac{G_{2}}{z^{2}}+\mathcal{O}\left(z^{-3}\right)\right), \quad \text { as } z \rightarrow \infty
$$


with

$$
G_{1}=\left(\begin{array}{cc}
-N m_{1} & 0 \\
0 & N m_{1}
\end{array}\right), \quad G_{2}=\left(\begin{array}{cc}
\frac{N^{2} m_{1}^{2}-N m_{2}}{2} & 0 \\
0 & \frac{N^{2} m_{1}^{2}+N m_{2}}{2}
\end{array}\right) .
$$

By (3.12), $m_{1}$ and $m_{2}$ can be computed explicitly, we have

$$
m_{1}=\frac{a+b}{2}=\frac{r}{2}, \quad m_{2}=\frac{1}{16}\left(5 a^{2}+6 a b+5 b^{2}\right)=\frac{1}{4}\left(r^{2}+\sigma\right) .
$$

\subsection{Transformation $Y \mapsto T$}

We use the $g$-function in the first transformation of the $\mathrm{RH}$ problem and define $T$ by

$$
T(z)=e^{-N \ell \sigma_{3} / 2} Y(z) e^{-N(g(z)-\ell / 2) \sigma_{3}},
$$

where $Y$ is given by 3.26 , with $w(x)=x^{\alpha} e^{-N W(x)}$. From the RH conditions for $Y$, it is straightforward to obtain a $\mathrm{RH}$ problem for the new unknown $T$ : we have

\section{RH problem for $T$}

(a) $T$ is analytic in $\mathbb{C} \backslash[0,+\infty)$.

(b) For $x \in \mathbb{R}$, we have the jump relation

$$
T_{+}(x)=T_{-}(x) J_{T}(x), \quad x \in[a, b],
$$

with

$$
J_{T}(x)=\left(\begin{array}{cc}
e^{-N\left(g_{+}(x)-g_{-}(x)\right)} & x^{\alpha} e^{N\left(g_{+}(x)+g_{-}(x)-W(x)-\ell\right)} \\
0 & e^{N\left(g_{+}(x)-g_{-}(x)\right)}
\end{array}\right) .
$$

(c) As $z \rightarrow \infty, T(z)=I+\mathcal{O}\left(z^{-1}\right)$.

Condition (c) follows from the logarithmic behavior of $g$ at infinity, condition (b) is a consequence of (4.9) and the jump relation (3.27) for $Y$. It is useful to define $\phi(z)$ as follows:

$$
\phi(z)=2 g(z)-W(z)-\ell, \quad z \in \mathbb{C} \backslash(-\infty, b] .
$$

From (4.3), it follows that

$$
\begin{array}{ll}
g_{+}(x)-g_{-}(x)=\phi_{+}(x), & x \in[a, b], \\
g_{+}(x)-g_{-}(x)=-\phi_{-}(x), & x \in[a, b] .
\end{array}
$$

It turns out that $\phi$ can be evaluated explicitly: we have

$$
\begin{aligned}
\phi(z)=\frac{-4}{\sigma}\left(-\frac{(b-a)^{2}}{4} \log [\sqrt{z-a}+\sqrt{z-b}]\right. \\
\left.+\frac{2 z-(a+b)}{4} \sqrt{z-b} \sqrt{z-a}+\frac{(b-a)^{2} \log (b-a)}{8}\right) .
\end{aligned}
$$

Using (4.2) and (4.3), we can write the jump matrix $J_{T}$ in terms of $\phi$ :

$$
\begin{array}{ll}
J_{T}(x)=\left(\begin{array}{cc}
e^{-N \phi_{+}(x)} & x^{\alpha} \\
0 & e^{-N \phi_{-}(x)}
\end{array}\right), & \text { for } x \in[a, b], \\
J_{T}(x)=\left(\begin{array}{cc}
1 & x^{\alpha} e^{N \phi(x)} \\
0 & 1
\end{array}\right), & \text { for } x \in(0, a) \cup(b,+\infty) .
\end{array}
$$

Note that, although $\phi$ is not continuous on $(0, a), e^{N \phi}$ is, and therefore we can safely write $e^{N \phi}$ instead of $e^{N \phi_{ \pm}}$. 


\subsection{Transformation $T \mapsto S$}

The jump matrix for $T$ can be re-written for $x \in(a, b)$ as

$$
J_{T}(x)=\left(\begin{array}{cc}
1 & 0 \\
\frac{e^{-N \phi_{-}(x)}}{x^{\alpha}} & 1
\end{array}\right)\left(\begin{array}{cc}
0 & x^{\alpha} \\
-\frac{1}{x^{\alpha}} & 0
\end{array}\right)\left(\begin{array}{cc}
1 & 0 \\
\frac{e^{-N \phi_{+}(x)}}{x^{\alpha}} & 1
\end{array}\right) .
$$

This leads us to the second transformation, where we open lenses. Define three regions as follows, see Figure 2:

- Region I: the upper lens region.

- Region II: the lower lens region.

- Region III: everything else.

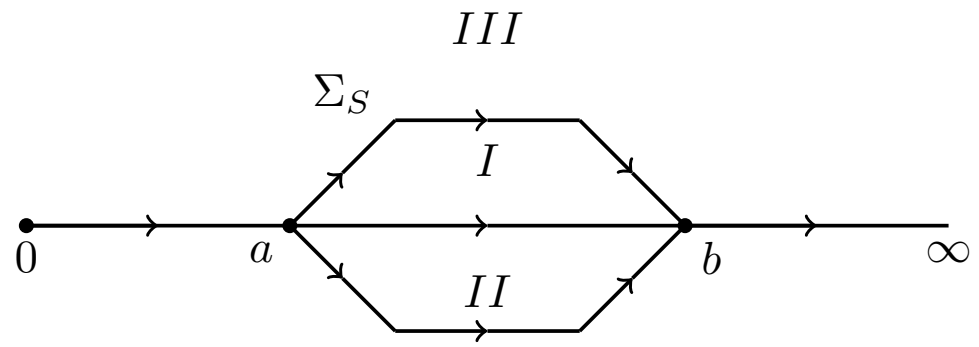

\section{$I I I$}

Figure 2: The contour $\Sigma_{S}$ consists of the two lenses and the interval $(0, \infty)$.

We then define $S$ in the three regions as follows:

$$
S(z)=T(z) \times \begin{cases}I & z \in \text { Region III, } \\
\left(\begin{array}{cc}
1 & 0 \\
-\frac{e^{-N \phi_{+}(z)}}{z^{\alpha}} & 1
\end{array}\right) & z \in \text { Region I, } \\
\left(\begin{array}{cc}
1 & 0 \\
\frac{e^{-N \phi_{-}(z)}}{z^{\alpha}} & 1
\end{array}\right) & z \in \text { Region II. }\end{cases}
$$

Using the RH conditions satisfied by $T$, we can deduce the $\mathrm{RH}$ problem for $S$.

\section{RH problem for $S$}

(a) $S$ is analytic in $\mathbb{C} \backslash \Sigma_{S}$, where $\Sigma_{S}$ is the contour shown in Figure 2 .

(b) The jump relations for $S$ are

$$
S_{+}(z)=S_{-}(z) J_{S}(z), \quad z \in \Sigma_{S},
$$


where

$$
\begin{array}{ll}
J_{S}(z)=\left(\begin{array}{cc}
1 & 0 \\
\frac{e^{-N \phi(z)}}{z^{\alpha}} & 1
\end{array}\right), & z \in \text { lower lens boundary, } \\
J_{S}(z)=\left(\begin{array}{cc}
0 & z^{\alpha} \\
-\frac{1}{z^{\alpha}} & 0
\end{array}\right), & z \in(a, b), \\
J_{S}(z)=\left(\begin{array}{cc}
1 & 0 \\
\frac{e^{-N \phi(z)}}{z^{\alpha}} & 1
\end{array}\right), & z \in \text { upper lens boundary, } \\
J_{S}(z)=J_{T}(z), & z \in(0, a) \cup(b,+\infty) .
\end{array}
$$

(c) $S(z)=I+\mathcal{O}\left(z^{-1}\right)$ as $z \rightarrow \infty$.

Using the explicit formula for $\phi$ given in 4.15), one can verify that the jump matrix for $S$ is exponentially small as $N \rightarrow \infty$ on $\Sigma_{S} \backslash[a, b]$, uniformly for $z$ bounded away from the endpoints $a$ and $b$. The next critical step is to build approximations to $S$ in 4 regions: a neighborhood of $z=b$, a neighborhood of $z=a$, a neighborhood of $z=0$, and the complement of those three regions. We will call the parametrix in the latter region "the outer parametrix" and this one will take care of the jump on $(a, b)$. The local parametrices near $a$ and $b$ will deal with the jumps near $a$ and $b$, where the uniform convergence breaks down. The local parametrix near 0 finally is needed for technical reasons because 0 is an endpoint of the jump contour $\Sigma_{S}$.

\subsection{The outer Parametrix $P^{\infty}(z)$}

We seek for a function $P^{\infty}(z)$ which satisfies the same $\mathrm{RH}$ conditions as $S$, but where we ignore the jumps that are small in the large $n$ limit and the jumps in small neighborhoods of $a$ and $b$. We thus obtain

\section{RH problem for $P^{\infty}(z)$}

(a) $P^{\infty}$ is analytic in $\mathbb{C} \backslash[a, b]$.

(b) For $z \in(a, b)$, we have

$$
P_{+}^{\infty}(z)=P_{-}^{\infty}(z)\left(\begin{array}{cc}
0 & z^{\alpha} \\
-\frac{1}{z^{\alpha}} & 0
\end{array}\right) .
$$

(c) $P^{\infty}(z)=I+\mathcal{O}\left(z^{-1}\right)$ as $z \rightarrow \infty$.

This RH problem is solved by a function of the form

$$
P^{\infty}(z)=d^{\sigma_{3}} P_{0}^{\infty}(z) D(z)^{-\sigma_{3}}
$$

where

$$
P_{0}^{\infty}(z)=\left(\begin{array}{ll}
\frac{\gamma(z)+\gamma(z)^{-1}}{2} & \frac{\gamma(z)-\gamma(z)^{-1}}{2 i} \\
\frac{\gamma(z)-\gamma(z)^{-1}}{-2 i} & \frac{\gamma(z)+\gamma(z)^{-1}}{2}
\end{array}\right),
$$

and

$$
\gamma(z)=\frac{(z-b)^{1 / 4}}{(z-a)^{1 / 4}}
$$


The quantity $D(z)$ is given by

$$
D(z)=\exp \left\{\frac{\alpha \sqrt{z-b} \sqrt{z-a}}{2 \pi i} \int_{a}^{b} \frac{\log x}{\sqrt{x-b} \sqrt{x-a}} \frac{d x}{x-z}\right\},
$$

and can be computed explicitly as follows:

$$
D(z)=\left(\frac{\sqrt{b(z-a)}+\sqrt{a(z-b)}}{\sqrt{z-a}+\sqrt{z-b}}\right)^{\alpha} .
$$

The constant $d$ is the large $z$ limit of $D(z)$ :

$$
d=\left(\frac{\sqrt{b}+\sqrt{a}}{2}\right)^{\alpha}
$$

It will be useful to have the expansion of $P^{\infty}$ for $z \rightarrow \infty$. For that, we will also need the asymptotic behavior of $D(z)$ and $P^{\infty}(z)$ as $z \rightarrow \infty$ : we have

$$
D(z)=d\left(1+\frac{d_{1}}{z}+\frac{d_{2}}{z^{2}}+\mathcal{O}\left(z^{-3}\right)\right)
$$

and

$$
P_{0}^{\infty}(z)=I+\frac{1}{z}\left(\begin{array}{cc}
0 & \frac{i(b-a)}{4} \\
\frac{-i(b-a)}{4} & 0
\end{array}\right)+\frac{1}{z^{2}}\left(\begin{array}{cc}
\frac{(b-a)^{2}}{32} & \frac{i\left(b^{2}-a^{2}\right)}{8} \\
\frac{-i\left(b^{2}-a^{2}\right)}{8} & \frac{(b-a)^{2}}{32}
\end{array}\right)+\mathcal{O}\left(z^{-3}\right) .
$$

Hence, after straightforward calculations, we obtain

$$
P^{\infty}(z)=I+\frac{P_{1}(\alpha)}{z}+\frac{P_{2}(\alpha)}{z^{2}}+\mathcal{O}\left(z^{-3}\right), \quad z \rightarrow \infty,
$$

with

$$
\begin{aligned}
& P_{1}(\alpha)=\left(\begin{array}{cc}
-d_{1} & \frac{i d^{2}(b-a)}{4} \\
\frac{-i(b-a)}{4 d^{2}} & d_{1}
\end{array}\right), \\
& P_{2}(\alpha)=\left(\begin{array}{cc}
\frac{(b-a)^{2}}{32}+d_{1}^{2}-d_{2} & \frac{i d^{2}(b-a)\left(a+b+2 d_{1}\right)}{8} \\
\frac{-i(b-a)\left(a+b-2 d_{1}\right)}{8 d^{2}} & \frac{(b-a)^{2}}{32}+d_{2}
\end{array}\right),
\end{aligned}
$$

where $d$ is defined in 4.31) and $d_{1}$ and $d_{2}$ are defined in 4.32). We have

$$
\begin{aligned}
& d_{1}(\alpha)=\frac{\alpha}{4}(\sqrt{b}-\sqrt{a})^{2}, \\
& d_{2}(\alpha)=\frac{d_{1}(\alpha)}{8}\left(3 a+2 \sqrt{a b}+3 b+\alpha(\sqrt{b}-\sqrt{a})^{2}\right) .
\end{aligned}
$$

Note that the outer parametrix $P^{\infty}$ depends on $\sigma$ through $a=a_{\sigma}$ and $b=b_{\sigma}$, and so do $D$, the constants $d, d_{1}, d_{2}$, and the matrices $P_{1}$ and $P_{2}$. 


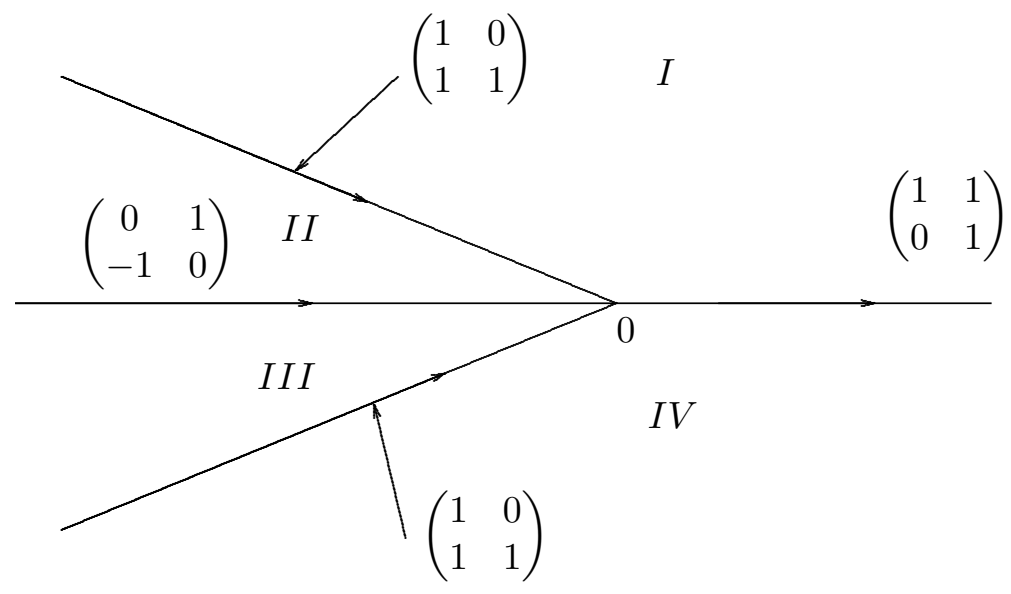

Figure 3: The jump contour $\Gamma$ for $\Psi$.

\subsection{The Airy Parametrices}

Recall that $a$ and $b$ depend on $r$ and $\sigma$; if $\sigma=s_{ \pm}$with $s_{ \pm}$given by (2.6), $a$ and $b$ will thus depend on $N$. However, for $N$ large, $\sigma$ will be close to $s$, and $a, b$ will be close to $\frac{1}{2}(r \mp 2 \sqrt{s})$. We will construct local parametrices in fixed sufficiently small disks $B_{ \pm}:=B_{\delta}\left(\frac{1}{2}(r \pm 2 \sqrt{s})\right)$ centered at $\frac{1}{2}(r \mp 2 \sqrt{s})$. For $N$ sufficiently large, those neighborhoods will also contain the points $a$ and $b$. The parametrices will be built out of a well-known Airy model RH problem, which we recall here. Denote

$$
\omega:=e^{\frac{2 \pi i}{3}}
$$

and let the complex $\zeta$ plane be divided into 4 regions as shown in Figure 3 . Define

$$
\Psi: \mathbb{C} \backslash \Gamma \rightarrow \mathbb{C}^{2 \times 2},
$$

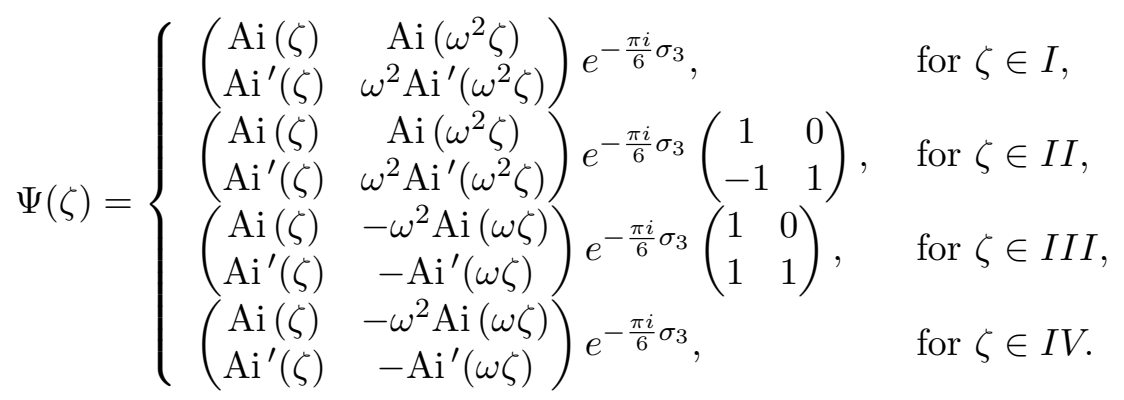

Then $\Psi$ satisfies the following model RH problem.

\section{RH problem for $\Psi$}

(a) $\Psi$ is analytic in the complex plane, except on the jump contour $\Gamma$ shown in Figure 3.

(b) For $\zeta$ on one of the four rays in the jump contour, we have $\Psi_{+}(\zeta)=\Psi_{-}(\zeta) J(\zeta)$, with $J(\zeta)$ the piecewise constant matrix indicated in Figure 3. 
(b) As $\zeta \rightarrow \infty$, we have

$$
\Psi(\zeta) e^{\frac{2}{3} \zeta^{3 / 2} \sigma_{3}}=\frac{e^{\frac{\pi i}{12}}}{2 \sqrt{\pi}}\left(\begin{array}{cc}
\zeta^{-1 / 4} & 0 \\
0 & \zeta^{1 / 4}
\end{array}\right) \sum_{k=0}^{\infty}\left(\begin{array}{cc}
(-1)^{k} s_{k} & s_{k} \\
-(-1)^{k} r_{k} & r_{k}
\end{array}\right) e^{-\frac{\pi i}{4} \sigma_{3}}\left(\frac{2}{3} \zeta^{3 / 2}\right)^{-k}
$$

where

$$
s_{k}=\frac{\Gamma(3 k+1 / 2)}{54^{k} k ! \Gamma(k+1 / 2)}, \quad r_{k}=-\frac{6 k+1}{6 k-1} s_{k} \quad \text { for } k \geq 1 .
$$

For future reference:

$$
\begin{aligned}
& s_{1}=\frac{5}{72}, \quad r_{1}=-\frac{7}{72} \\
& s_{2}=\frac{385}{10368}, \quad r_{2}=-\frac{454}{10368} .
\end{aligned}
$$

The following alternative version of the asymptotic behavior of $\Psi$ as $\zeta \rightarrow \infty$ will be useful,

$$
\begin{aligned}
& \Psi(\zeta)=\zeta^{-\frac{\sigma_{3}}{4}} \frac{e^{\frac{i \pi}{12}}}{2 \sqrt{\pi}}\left(\begin{array}{cc}
1 & 1 \\
-1 & 1
\end{array}\right) \\
& \times\left[I+\sum_{k=1}^{\infty} \frac{1}{2}\left(\frac{2}{3} \zeta^{3 / 2}\right)^{-k}\left(\begin{array}{cc}
(-1)^{k}\left(s_{k}+r_{k}\right) & s_{k}-r_{k} \\
(-1)^{k}\left(s_{k}-r_{k}\right) & s_{k}+r_{k}
\end{array}\right)\right] e^{-\frac{\pi i}{4} \sigma_{3}} e^{-\frac{2}{3} \zeta^{3 / 2} \sigma_{3}} \\
& =\frac{e^{\frac{i \pi}{12}} \zeta^{-\frac{\sigma_{3}}{4}}}{2 \sqrt{\pi}}\left(\begin{array}{cc}
1 & 1 \\
-1 & 1
\end{array}\right) \\
& \times\left[I+\frac{1}{8 \zeta^{3 / 2}}\left(\begin{array}{cc}
\frac{1}{6} & 1 \\
-1 & -\frac{1}{6}
\end{array}\right)+\frac{35}{384 \zeta^{3}}\left(\begin{array}{cc}
\frac{1}{12} & 1 \\
-1 & -\frac{1}{12}
\end{array}\right)+\cdots\right] e^{-\frac{\pi i}{4} \sigma_{3}} e^{-\frac{2}{3} \zeta^{3 / 2} \sigma_{3}} .
\end{aligned}
$$

We define the function

$$
f_{b}(z)=\left(\frac{3}{4}\right)^{2 / 3}(-\phi(z))^{2 / 3}, \quad z \in B_{+},
$$

where $\phi$ is defined in 4.12 . By 4.15 we obtain that $\phi$ behaves like

$$
\phi(z) \sim-\frac{8 \sqrt{b-a}}{3 \sigma}(z-b)^{3 / 2}, \quad z \rightarrow b,
$$

so that we can take $f_{b}$ to be analytic near $b$, and particularly in the disk $B_{+}$. We then have

$$
f_{b}(z)=\left(\frac{2 \sqrt{b-a}}{\sigma}\right)^{2 / 3}(z-b)\left(1+\frac{z-b}{5(b-a)}+\mathcal{O}\left((z-b)^{2}\right)\right), \quad z \rightarrow b .
$$

The next auxiliary quantity which we define is the analytic matrix-valued function $E_{b}(z)$ for $z \in B_{+}$, defined as follows,

$$
E_{b}(z)=\sqrt{\pi} e^{\frac{-i \pi}{12}} P^{(\infty)}(z) z^{\alpha \sigma_{3} / 2} e^{\frac{\pi i}{4} \sigma_{3}}\left(\begin{array}{cc}
1 & -1 \\
1 & 1
\end{array}\right) N^{\frac{\sigma_{3}}{6}} f_{b}(z)^{\sigma_{3} / 4}
$$


This function is analytic near $b$, we can see this for example by observing that it has no jump across the interval $(b-\delta, b)$ :

$$
\begin{aligned}
& \left(E_{b}\right)_{-}^{-1}\left(E_{b}\right)_{+}=\left(f_{b}^{-\sigma_{3} / 4}\right)_{-} \frac{1}{2}\left(\begin{array}{cc}
1 & 1 \\
-1 & 1
\end{array}\right) e^{-\frac{\pi i}{4} \sigma_{3}} z^{-\alpha \sigma_{3} / 2} \times \\
& \times\left(P^{\infty}\right)_{-}^{-1}\left(P^{\infty}\right)_{+} z^{\alpha \sigma_{3} / 2} e^{\frac{\pi i}{4} \sigma_{3}}\left(\begin{array}{cc}
1 & -1 \\
1 & 1
\end{array}\right)\left(f_{b}^{\sigma_{3} / 4}\right)_{+} \\
& =\left(f_{b}^{-\sigma_{3} / 4}\right)_{-} \frac{1}{2}\left(\begin{array}{cc}
1 & 1 \\
-1 & 1
\end{array}\right) e^{-\frac{\pi i}{4} \sigma_{3}}\left(\begin{array}{cc}
0 & 1 \\
-1 & 0
\end{array}\right) e^{\frac{\pi i}{4} \sigma_{3}}\left(\begin{array}{cc}
1 & -1 \\
1 & 1
\end{array}\right)\left(f_{b}^{\sigma_{3} / 4}\right)_{+} \\
& =\left(f_{b}^{-\sigma_{3} / 4}\right)_{-} \frac{1}{2}\left(\begin{array}{cc}
1 & 1 \\
-1 & 1
\end{array}\right)\left(\begin{array}{cc}
0 & -i \\
-i & 0
\end{array}\right)\left(\begin{array}{cc}
1 & -1 \\
1 & 1
\end{array}\right)\left(f_{b}^{\sigma_{3} / 4}\right)_{+} \\
& =\left(f_{b}^{-\sigma_{3} / 4}\right)_{-}\left(\begin{array}{cc}
-i & 0 \\
0 & i
\end{array}\right)\left(f_{b}^{\sigma_{3} / 4}\right)_{+}=\left(\begin{array}{cc}
1 & 0 \\
0 & 1
\end{array}\right) .
\end{aligned}
$$

Within the neighborhood $B_{+}$of $b$, we define

$$
P^{(b)}(z)=E_{b}(z) \Psi\left(N^{2 / 3} f_{b}(z)\right) e^{2 N \sigma_{3} f_{b}^{3 / 2}(z) / 3} z^{-\frac{\alpha}{2} \sigma_{3}}, \quad \text { for } z \in B_{+} .
$$

The local parametrix $P^{(b)}$ then satisfies the following conditions.

\section{RH problem for $P^{(b)}$}

(a) $P^{(b)}$ is analytic in $\overline{B_{+}} \backslash \Sigma_{S}$,

(b) $P_{+}^{(b)}(z)=P_{-}^{(b)}(z) J_{S}(z)$ for $z \in B_{+} \cap \Sigma_{S}$,

(c) for $z \in \partial B_{+}$, we have

$$
\begin{aligned}
& P^{(b)}(z)=P^{\infty}(z) z^{\alpha \sigma_{3} / 2} e^{\frac{\pi i}{4} \sigma_{3}} \\
& \times\left[I+\sum_{k=1}^{\infty} \frac{1}{2}\left(\frac{2 N}{3} f_{b}(z)^{3 / 2}\right)^{-k}\left(\begin{array}{cc}
(-1)^{k}\left(s_{k}+r_{k}\right) & s_{k}-r_{k} \\
(-1)^{k}\left(s_{k}-r_{k}\right) & s_{k}+r_{k}
\end{array}\right)\right] \\
& \times e^{-\frac{\pi i}{4} \sigma_{3}} z^{-\frac{\alpha}{2} \sigma_{3}}
\end{aligned}
$$

as $N \rightarrow \infty$.

The construction of the parametrix in a neighborhood of the point $a$ is similar. First, we define the transformation $f_{a}(z)$ for $z \in B_{-}$, analogous to $f_{b}$ defined in 4.48 above,

$$
f_{a}(z) \equiv\left(\frac{3}{4}\right)^{2 / 3}(\phi(a)-\phi(z))^{2 / 3}, \quad z \in B_{\delta}\left(a_{s}\right)
$$

As $z \rightarrow a$, by (4.15) we may choose the branch so that $f_{a}(z)$ is analytic in a neighborhood of $a$, and

$$
f_{a}(z)=-\left(2 \frac{\sqrt{b-a}}{\sigma}\right)^{2 / 3}(z-a)\left(1-\frac{z-a}{5(b-a)}+\mathcal{O}\left((z-a)^{2}\right)\right), \quad z \rightarrow a .
$$


Next we define $E_{a}(z)$ as follows:

$$
E_{a}(z)=e^{\frac{-\pi i}{12}} \sqrt{\pi} P^{\infty}(z) \sigma_{3} z^{\frac{\alpha}{2} \sigma_{3}} e^{\frac{\pi i}{4} \sigma_{3}}\left(\begin{array}{cc}
1 & -1 \\
1 & 1
\end{array}\right) N^{\frac{\sigma_{3}}{6}} f_{a}(z)^{\sigma_{3} / 4}
$$

We now define the approximation $P^{(a)}$ within $B_{-}$:

$$
P^{(a)}(z)=E_{a}(z) \Psi\left(N^{2 / 3} f_{a}(z)\right) e^{2 n \sigma_{3} f_{a}^{3 / 2}(z) / 3} z^{-\frac{\alpha}{2} \sigma_{3}} \sigma_{3}, \quad \text { for } z \in B_{-} .
$$

We then have

\section{RH problem for $P^{(a)}$}

(a) $P^{(a)}$ is analytic in $\overline{B_{-}} \backslash \Sigma_{S}$,

(b) $P_{+}^{(a)}(z)=P_{-}^{(a)}(z) J_{S}(z)$ for $z \in B_{-} \cap \Sigma_{S}$,

(c) for $z \in \partial B_{-}$, we have

$$
\begin{aligned}
& P^{(a)}(z)=P^{\infty}(z) z^{\alpha \sigma_{3} / 2} e^{\frac{\pi i}{4} \sigma_{3}} \\
& \times\left[I+\sum_{k=1}^{\infty} \frac{1}{2}\left(\frac{2 N}{3} f_{a}(z)^{3 / 2}\right)^{-k}\left(\begin{array}{cc}
(-1)^{k}\left(s_{k}+r_{k}\right) & -\left(s_{k}-r_{k}\right) \\
-(-1)^{k}\left(s_{k}-r_{k}\right) & s_{k}+r_{k}
\end{array}\right)\right] \times \\
& \times e^{-\frac{\pi i}{4} \sigma_{3}} z^{-\frac{\alpha}{2} \sigma_{3}}
\end{aligned}
$$

as $N \rightarrow \infty$.

\subsection{The parametrix near 0}

In a fixed neighborhood $B_{0}$ of 0 , we would like to construct a local parametrix $P^{(0)}$ which satisfies the following $\mathrm{RH}$ conditions.

\section{RH problem for $P^{(0)}$}

(a) $P^{(0)}$ is analytic in $\overline{B_{0}} \backslash \Sigma_{S}$,

(b) $P_{+}^{(0)}(z)=P_{-}^{(0)}(z) J_{S}(z)$ for $z \in B_{0} \cap \Sigma_{S}$,

(c) for $z \in \partial B_{0}$, we have

$$
P^{(0)}(z)=P^{\infty}(z)\left(I+\mathcal{O}\left(e^{-c N}\right)\right), \quad N \rightarrow \infty .
$$

The jump relation should in other words be

$$
P_{+}^{(0)}(x)=P_{-}^{(0)}(x)\left(\begin{array}{cc}
1 & x^{\alpha} e^{N \phi(x)} \\
0 & 1
\end{array}\right) \quad 0<x<a .
$$

A solution to this problem is given by

$$
P^{(0)}(z)=P^{\infty}(z)\left(\begin{array}{cc}
1 & \frac{\left(z^{\alpha}-1\right)}{1-e^{2 \pi i \alpha}} e^{N \phi(z)} \\
0 & 1
\end{array}\right),
$$

where the function $z^{\alpha}$ is chosen with branch cut on the positive half-line, $z^{\alpha}=|z|^{\alpha} e^{i \alpha \theta}$, $0 \leq \theta<2 \pi$. It is important to note that as $\alpha \rightarrow 0$, the limit of $P^{(0)}(z)$ exists for $z \neq 0$, and that it is given by

$$
P^{(0)}(z)=P^{\infty}(z)\left(\begin{array}{cc}
1 & -\frac{\log z}{2 \pi i} e^{N \phi(z)} \\
0 & 1
\end{array}\right) .
$$




\subsection{RH problem for the error, $R$}

We now define $R$ in four regions of the plane, using our approximations to $S$. Set

$$
R(z)= \begin{cases}S(z)\left(P^{(0)}(z)\right)^{-1}, & z \in B_{0} \\ S(z)\left(P^{(a)}(z)\right)^{-1}, & z \in B_{-} \\ S(z)\left(P^{(b)}(z)\right)^{-1}, & z \in B_{+} \\ S(z)\left(P^{\infty}(z)\right)^{-1}, & \text { everywhere else. }\end{cases}
$$

The quantity $R$ is piecewise analytic, with jumps across the contours $\Sigma_{j}, j=1, \ldots, 7$, as shown in Figure 4.

\section{RH problem for $R$}

(a) $R$ is analytic in $\mathbb{C} \backslash \cup_{j=1}^{7} \Sigma_{j}$,

(b) For $z \in \cup_{j=1}^{7} \Sigma_{j}$, we have $R_{+}(z)=R_{-}(z) J_{R}(z)$, with

$$
\begin{aligned}
J_{R}(z) & =P^{\infty}(z) J_{S}(z)\left(P^{\infty}(z)\right)^{-1}, & z \in \Sigma_{1} \cup \Sigma_{2} \cup \Sigma_{3} \cup \Sigma_{4}, \\
J_{R}(z) & =P^{(0)}(z)\left(P^{\infty}(z)\right)^{-1}, & z \in \Sigma_{5}, \\
J_{R}(z) & =P^{(a)}(z)\left(P^{\infty}(z)\right)^{-1}, & z \in \Sigma_{6}, \\
J_{R}(z) & =P^{(b)}(z)\left(P^{\infty}(z)\right)^{-1}, & z \in \Sigma_{7} .
\end{aligned}
$$

(c) As $z \rightarrow \infty$, we have

$$
R(z)=I+\mathcal{O}\left(z^{-1}\right)
$$

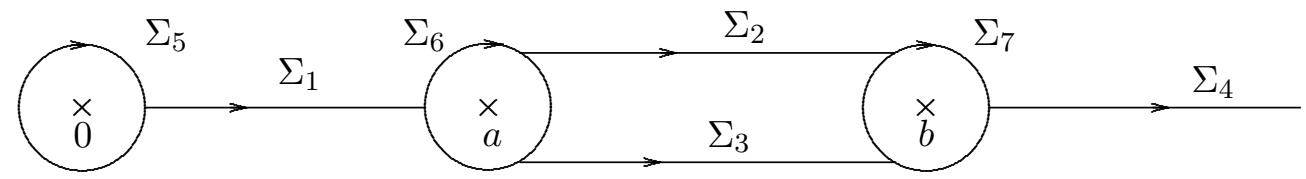

Figure 4: The jump contour for $R$.

The jump matrices across the contours $\Sigma_{1}, \Sigma_{2}, \Sigma_{3}$, and $\Sigma_{4}$ are all exponentially close to $I$ for large $N$ because $J_{S}$ is exponentially small and $P^{\infty}$ and its inverse are bounded on the contour, which does not contain the points $a$ and $b$. The jump across $\Sigma_{5}=\partial B_{0}$ is also shown to be exponentially small by 4.59$)$, since $e^{N \phi(z)}$ is exponentially small for $z$ near 0 . The only jumps that are not exponentially small are the ones on $\Sigma_{6}=\partial B_{-}$and $\Sigma_{7}=\partial B_{+}$. There we have for any $K$, by (4.58) and 4.53),

$$
J_{R}(z)=I+\sum_{k=1}^{K} J^{(k)}(z, \alpha) N^{-k}+\mathcal{O}\left(N^{-K-1}\right), \quad N \rightarrow \infty .
$$

By 4.58 and 4.53, we have

$$
\begin{aligned}
J^{(k)}(z, \alpha)= & \frac{1}{2}\left(\frac{2}{3} f_{a}(z)^{3 / 2}\right)^{-k} \\
& \times P^{\infty}(z)\left(\begin{array}{cc}
(-1)^{k}\left(s_{k}+t_{k}\right) & -i z^{\alpha}\left(s_{k}-r_{k}\right) \\
(-1)^{k} i z^{-\alpha}\left(s_{k}-r_{k}\right) & s_{k}+r_{k}
\end{array}\right)\left(P^{\infty}(z)\right)^{-1}
\end{aligned}
$$


for $z \in \Sigma_{6}$, and

$$
\begin{aligned}
J^{(k)}(z, \alpha)= & \frac{1}{2}\left(\frac{2}{3} f_{b}(z)^{3 / 2}\right)^{-k} \\
& \times P^{\infty}(z)\left(\begin{array}{cc}
(-1)^{k}\left(s_{k}+r_{k}\right) & i z^{\alpha}\left(s_{k}-r_{k}\right) \\
-i(-1)^{k} z^{-\alpha}\left(s_{k}-r_{k}\right) & s_{k}+r_{k}
\end{array}\right)\left(P^{\infty}(z)\right)^{-1}
\end{aligned}
$$

for $z \in \Sigma_{7}$. Note that $J^{(1)}$ is a meromorphic function in $B_{-} \cup B_{+}$with double poles at $a$ and $b$.

Using standard estimates, it can then be proved that

$$
R(z)=I+\sum_{k=1}^{K} R^{(k)}(z, \alpha) N^{-k}+\mathcal{O}\left(N^{-K-1}\right), \quad N \rightarrow \infty,
$$

uniformly for $z$ off the jump contour, where $R^{(k)}$ is analytic except on $\Sigma_{6} \cup \Sigma_{7}$. Substituting 4.69 and $(4.72)$ into the jump relation $R_{+}=R_{-} J_{R}$ leads us to the following jump relation for $R^{(1)}$ :

$$
R_{+}^{(1)}(z, \alpha)-R_{-}^{(1)}(z, \alpha)=J^{(1)}(z, \alpha), \quad \text { for } z \in \Sigma_{6} \cup \Sigma_{7} .
$$

In addition, since $R \rightarrow I$ at infinity, $R^{(1)}(z, \alpha) \rightarrow 0$ as $z \rightarrow \infty$. These conditions determine $R^{(1)}$ uniquely, and we obtain

$$
\begin{array}{lrl}
R^{(1)}(z) & =Q(z, \alpha), & z \in \mathbb{C} \backslash\left(\overline{B_{-}} \cup \overline{B_{+}}\right), \\
R^{(1)}(z)=-J^{(1)}(z, \alpha)+Q(z, \alpha), & z \in B_{-}, \\
R^{(1)}(z)=-J^{(1)}(z, \alpha)+Q(z, \alpha), & z \in B_{+},
\end{array}
$$

where

$$
\begin{aligned}
Q(z, \alpha)=\frac{1}{z-a} & \operatorname{Res}_{z=a} J^{(1)}(z, \alpha)+\frac{1}{(z-a)^{2}} \operatorname{Res}_{z=a}\left[(z-a) J^{(1)}(z, \alpha)\right] \\
& +\frac{1}{z-b} \operatorname{Res}_{z=b} J^{(1)}(z, \alpha)+\frac{1}{(z-b)^{2}} \operatorname{Res}_{z=b}\left[(z-b) J^{(1)}(z, \alpha)\right] .
\end{aligned}
$$

As $z \rightarrow \infty$, we have an asymptotic expansion of the form

$$
R(z)=I+\sum_{k=1}^{K} R_{k}(N, \alpha) z^{-k}+\mathcal{O}\left(z^{-K-1}\right), \quad z \rightarrow \infty .
$$

From the asymptotics 4.72 for $R$ and the formula 4.74 for $R^{(1)}$, we obtain

$$
\begin{aligned}
& R_{1}(N, \alpha)=\frac{1}{N}\left(\operatorname{Res}_{z=a} J^{(1)}(z, \alpha)+\operatorname{Res}_{z=b} J^{(1)}(z, \alpha)\right)+\mathcal{O}\left(N^{-2}\right), \\
& R_{2}(N, \alpha)=\frac{1}{N}\left(\operatorname{Res}_{z=a}\left[(z-a) J^{(1)}(z, \alpha)\right]+\operatorname{Res}_{z=b}\left[(z-b) J^{(1)}(z, \alpha)\right]\right) \\
& \quad+\frac{1}{N}\left(a \operatorname{Res}_{z=a} J^{(1)}(z, \alpha)+b \operatorname{Res}_{z=b} J^{(1)}(z, \alpha)\right)+\mathcal{O}\left(N^{-2}\right),
\end{aligned}
$$

as $N \rightarrow \infty$. Since the jump matrix $J_{R}$ depends analytically on the parameters $s>0$ and $r>2 \sqrt{s}$, one can deduce that the functions $R_{1}$ and $R_{2}$, and all of the coefficients in their large $N$ asymptotic expansions, depend analytically on $s$ and $r$. 
After a straightforward but long calculation, one obtains, writing $c_{1}=\sqrt{a}-\sqrt{b}$ and $d$ as in (4.31),

$$
\begin{aligned}
R_{1,11}(N, \alpha) & =-R_{1,22}(N, \alpha) \\
= & \left(\frac{\sigma}{32(b-a)}-\frac{\alpha^{2} \sigma}{16 b(b-a)}(\sqrt{a}-\sqrt{b})^{2}\right) \frac{1}{N}+\mathcal{O}\left(N^{-2}\right) \\
R_{1,12}(N, \alpha) & \\
= & \frac{i d(\alpha)^{2} \sigma}{48 b(b-a)}\left(3 \alpha^{2}(\sqrt{a}-\sqrt{b})^{2}-6 \alpha \sqrt{a b}+2 b+6 \alpha b\right) \frac{1}{N}+\mathcal{O}\left(N^{-2}\right) \\
R_{1,21}(N, \alpha) & =\frac{i \sigma}{48 b(b-a) d(\alpha)^{2}}\left(3 \alpha^{2}(\sqrt{a}-\sqrt{b})^{2}+6 \alpha \sqrt{a b}+2 b-6 \alpha b\right) \frac{1}{N} \\
R_{2,11}(N, \alpha) & =\frac{\sigma}{32}\left(-\frac{5}{6}+\frac{-2 \alpha^{2}(\sqrt{a}-\sqrt{b})^{2}+b}{b-a}\right) \frac{1}{N}+\mathcal{O}\left(N^{-2}\right)
\end{aligned}
$$

as $N \rightarrow \infty$.

\section{Proof of Theorem 2.2}

We will now use the large $N$ asymptotics obtained from the $\mathrm{RH}$ analysis to obtain asymptotics for the function $\mathcal{G}_{N}(\alpha ; r, \sigma)$ in $(3.41)$.

Let us first consider the quantities $\tilde{Y}(z)$ for $z \in B_{0}$, which we need to compute the last two terms in (3.41). From (3.36), 4.9), 4.19), 4.63), and 4.61), it follows that

$$
\begin{aligned}
\tilde{Y}(z) & =e^{N \ell \sigma_{3} / 2} R(z) P^{(0)}(z) e^{N(g(z)-\ell / 2) \sigma_{3}}\left(\begin{array}{cc}
1 & -\frac{w(z ; \alpha)}{1-e^{2 \pi i \alpha}} \\
0 & 1
\end{array}\right) \\
& =e^{N \ell \sigma_{3} / 2} R(z) P^{\infty}(z)\left(\begin{array}{cc}
1 & \frac{\left(z^{\alpha}-1\right)}{1-e^{2 \pi i \alpha}} e^{N \phi(z)} \\
0 & 1
\end{array}\right) e^{N(g(z)-\ell / 2) \sigma_{3}}\left(\begin{array}{cc}
1 & -\frac{w(z ; \alpha)}{1-e^{2 \pi i \alpha}} \\
0 & 1
\end{array}\right) .
\end{aligned}
$$

By 4.12 , we have

$$
\tilde{Y}(z)=e^{N \ell \sigma_{3} / 2} R(z) P^{\infty}(z)\left(\begin{array}{cc}
1 & \frac{-1}{1-e^{2 \pi i \alpha}} e^{N \phi(z)} \\
0 & 1
\end{array}\right) e^{N(g(z)-\ell / 2) \sigma_{3}}
$$

and in the large $N$ limit

$$
\begin{aligned}
& \tilde{Y}_{12}(0)=e^{-N g(0)} e^{N \ell}\left(1+\mathcal{O}\left(N^{-1}\right)\right) P_{12}^{\infty}(0), \\
& \tilde{Y}_{22}(0)=e^{-N g(0)}\left(1+\mathcal{O}\left(N^{-1}\right)\right) P_{22}^{\infty}(0) .
\end{aligned}
$$

For the first column of $Y$ near 0 , we have

$$
\begin{aligned}
& Y_{11}(z)=\left(1+\mathcal{O}\left(N^{-1}\right)\right) P_{11}^{\infty}(z) e^{N g(z)}, \\
& Y_{21}(z)=\left(1+\mathcal{O}\left(N^{-1}\right)\right) P_{21}^{\infty}(z) e^{N g(z)} e^{-N \ell} .
\end{aligned}
$$

Let us now compute the moments at infinity $Y_{1}$ and $Y_{2}$ defined by (3.28). For $z$ outside the lenses, we have

$$
\begin{aligned}
Y(z) & =e^{N \ell \sigma_{3} / 2} S(z) e^{N(g(z)-\ell / 2) \sigma_{3}} \\
& =e^{N \ell \sigma_{3} / 2} R(z) P^{\infty}(z) e^{N(g(z)-\ell / 2) \sigma_{3}} .
\end{aligned}
$$


By 4.6, 4.34, and 4.78, it follows that

$$
Y_{1}(\alpha)=e^{N \ell \sigma_{3} / 2}\left[P_{1}(\alpha)+G_{1}+R_{1}(\alpha)\right] e^{-N \ell \sigma_{3} / 2},
$$

and

$$
\begin{aligned}
Y_{2}(\alpha)=e^{N \ell \sigma_{3} / 2}\left[P_{2}(\alpha)+G_{2}+R_{2}(\alpha)+\left(P_{1}(\alpha)+R_{1}(\alpha)\right) G_{1}+\right. & \left.R_{1}(\alpha) P_{1}(\alpha)\right] \\
& \times e^{-N \ell \sigma_{3} / 2} .
\end{aligned}
$$

Entry-wise, we obtain as $N \rightarrow \infty$, by 4.82-4.87,

$$
\begin{aligned}
& Y_{1,11}(\alpha)=-N m_{1}-d_{1}+\frac{\left(b-2 \alpha^{2}(\sqrt{a}-\sqrt{b})^{2}\right) \sigma}{32 b(b-a) N}+\mathcal{O}\left(N^{-2}\right), \\
& e^{-N \ell} Y_{1,12}(\alpha)=i d^{2} \frac{b-a}{4} \\
& \quad+\frac{i d^{2} \sigma}{48 a b(b-a) N}\left(3 \alpha^{2}(\sqrt{a}-\sqrt{b})^{2}-6 \alpha \sqrt{a b}+2 b+6 \alpha b\right)+\mathcal{O}\left(N^{-2}\right), \\
& e^{N \ell} Y_{1,21}(\alpha)=-i \frac{b-a}{4 d^{2}} \\
& \quad+\frac{i \sigma}{48 b(b-a) d^{2}}\left(3 \alpha^{2}(\sqrt{a}-\sqrt{b})^{2}+6 \alpha \sqrt{a b}+2 b-6 \alpha b\right)+\mathcal{O}\left(N^{-2}\right), \\
& Y_{1,22}(\alpha)=-Y_{1,11}(\alpha) .
\end{aligned}
$$

For $Y_{2,11}$, we have

$$
\begin{aligned}
Y_{2,11}(\alpha)=\frac{m_{1}^{2}}{2} N^{2}-\left(\frac{m_{2}}{2}-m_{1} d_{1}\right) & N+\frac{(b-a)^{2}}{32}+d_{1}^{2}-d_{2} \\
& -\frac{m_{1} \sigma\left(-2 \alpha^{2}(\sqrt{a}-\sqrt{b})^{2}+b\right)}{32 b(b-a)}+\mathcal{O}\left(N^{-1}\right) .
\end{aligned}
$$

Recall from (3.41) that

$$
\mathcal{G}_{N}(\alpha)=T_{1}(\alpha)+T_{2}(\alpha)+T_{3}(\alpha)+T_{4}(\alpha)+T_{5}(\alpha)
$$

with

$$
\begin{aligned}
& T_{1}(\alpha)=\frac{\alpha}{2} \frac{Y_{1,12}^{\prime}}{Y_{1,12}}, \\
& T_{2}(\alpha)=2 \frac{N}{\sigma}\left(2 Y_{2,11}^{\prime}-r Y_{1,11}^{\prime}+\left(\operatorname{det} Y_{1}\right)^{\prime}\right), \\
& T_{3}(\alpha)=\frac{N}{2}\left(\frac{Y_{1,12}^{\prime}}{Y_{1,12}}-\frac{Y_{1,21}^{\prime}}{Y_{1,21}}\right), \\
& T_{4}(\alpha)=\sqrt{Y_{1,12} Y_{1,21}}\left(\frac{Y_{11}(0 ; \alpha)}{\sqrt{-i Y_{1,12}}}\right)^{\prime} \frac{\alpha}{\sqrt{i Y_{1,21}}} \tilde{Y}_{22}(0 ; \alpha), \\
& T_{5}(\alpha)=-\sqrt{Y_{1,12} Y_{1,21}}\left(\frac{Y_{21}(0 ; \alpha)}{\sqrt{i Y_{1,21}}}\right)^{\prime} \frac{\alpha}{\sqrt{-i Y_{1,12}}} \tilde{Y}_{12}(0 ; \alpha) .
\end{aligned}
$$

We can now substitute (5.2)-(5.5) and (5.9)- $-(5.15)$ into (5.17)-(5.21) to obtain asymptotics for $T_{1}(\alpha), \ldots, T_{5}(\alpha)$. Their expressions can be computed further by the formulas 
4.79-4.86). We used Mathematica for this lengthy but straightforward calculation and obtain

$$
\begin{aligned}
& T_{1}=\alpha \frac{d^{\prime}(\alpha)}{d(\alpha)}+\mathcal{O}\left(N^{-1}\right), \\
& T_{2}=\left(r d_{1}^{\prime}(\alpha)+2 d_{1}(\alpha) d_{1}^{\prime}(\alpha)-2 d_{2}^{\prime}(\alpha)\right) \frac{2 N}{\sigma}-\frac{\alpha(\sqrt{a}-\sqrt{b})^{2}}{8 b}+\mathcal{O}\left(N^{-1}\right), \\
& T_{3}=2 \frac{d^{\prime}(\alpha)}{d(\alpha)} N+\frac{\alpha \sigma}{2 b(\sqrt{a}+\sqrt{b})^{2}}+\mathcal{O}\left(N^{-1}\right), \\
& T_{4}=\frac{\alpha}{4}\left(\gamma+\gamma^{-1}\right)^{2}\left(\frac{d^{\prime}(\alpha)}{d(\alpha)}-\frac{1}{2} \log (a b)\right)+\mathcal{O}\left(N^{-1}\right), \quad \gamma=b^{1 / 4} a^{-1 / 4}, \\
& T_{5}=-\frac{\alpha}{4}\left(\gamma-\gamma^{-1}\right)^{2}\left(\frac{d^{\prime}(\alpha)}{d(\alpha)}-\frac{1}{2} \log (a b)\right)+\mathcal{O}\left(N^{-1}\right) .
\end{aligned}
$$

Substituting 4.31), 4.37), 4.38), and $r=a+b$ (see (2.3), and summing up the expressions for $T_{1}, \ldots, T_{5}$, we obtain

$$
\mathcal{G}_{N}(\alpha ; r, \sigma)=\mathcal{G}^{(0)}(r, \sigma) N+\alpha \mathcal{G}^{(1)}(r, \sigma)+\mathcal{O}\left(N^{-1}\right),
$$

where

$$
\begin{aligned}
& \mathcal{G}^{(0)}(r, \sigma)=\frac{(\sqrt{b}-\sqrt{a})^{2}}{2(\sqrt{b}+\sqrt{a})^{2}}+2 \log \left(\frac{\sqrt{a}+\sqrt{b}}{2}\right), \\
& \mathcal{G}^{(1)}(r, \sigma)=2 \log \frac{\sqrt{a}+\sqrt{b}}{2(a b)^{1 / 4}} .
\end{aligned}
$$

It should be noted that all of the error terms we used above can be expanded in asymptotic series in negative integer powers of $N$, and this can be used to obtain a full asymptotic expansion for $\mathcal{G}_{N}(\alpha ; r, \sigma)$ :

$$
\mathcal{G}_{N}(\alpha ; r, \sigma)=\mathcal{G}^{(0)}(r, \sigma) N+\alpha \mathcal{G}^{(1)}(r, \sigma)+\sum_{j=1}^{k} f^{(j)}(\alpha ; r, \sigma) N^{-j}+\mathcal{O}\left(N^{-k-1}\right)
$$

for any $k \in \mathbb{N}$. The constants $f^{(j)}(\alpha ; r, \sigma)$ may depend on $\alpha$ and are real analytic functions in $r, \sigma$.

Let us now compute the asymptotics for $\log Z_{2 N}$ using $(3.49)$. Since the $\mathcal{O}(N)$-term in the expansion of $\mathcal{G}_{N}$ is independent of $\alpha$, the leading order terms of the two integrals in 3.49 cancel out against each other, and we obtain

$$
\log \left[\frac{1}{(2 N) !} Z_{2 N}\right]=2 \log \left[\frac{1}{N !} Z_{N, s}^{G U E}\right]+\frac{N^{2} r^{2}}{s}+\frac{1}{4} \mathcal{G}^{(1)}(r, s)+\mathcal{O}\left(N^{-1}\right)
$$

as $N \rightarrow \infty$. Replacing $2 N$ by $N$, we get the following large $N$ asymptotics for $N$ even:

$$
\log \left[\frac{1}{N !} Z_{N}\right]=2 \log \left[\frac{1}{(N / 2) !} Z_{N / 2, s}^{G U E}\right]+\frac{N^{2} r^{2}}{4 s}+\frac{1}{4} \mathcal{G}^{(1)}(r, s)+\mathcal{O}\left(N^{-1}\right)
$$


In order to have a more compact formula, we substract $2 \log \left[\frac{1}{(N / 2) !} Z_{N / 2, \sigma^{*}}^{G U E}\right]$, defined in 1.5 , at both sides of this formula, with $\sigma^{*}=4 e^{3 / 2}$, and obtain by 1.5 ,

$$
\begin{aligned}
\log \left[\frac{(N / 2) !^{2} Z_{N}}{N !\left(Z_{N / 2,4 e^{3 / 2}}^{G U E}\right)^{2}}\right] & =2 \log \left[\frac{Z_{N / 2, s}^{G U E}}{Z_{N / 2,4 e^{3 / 2}}^{G U E}}\right]+\frac{N^{2} r^{2}}{4 s}+\frac{1}{4} \mathcal{G}^{(1)}(r, s)+\mathcal{O}\left(N^{-1}\right) \\
& =-N^{2} F_{0}+\frac{1}{2} \log \frac{\sqrt{a}+\sqrt{b}}{2(a b)^{1 / 4}}+\mathcal{O}\left(N^{-1}\right)
\end{aligned}
$$

where

$$
F_{0}=-\frac{1}{4} \log \frac{s}{4}+\frac{3}{8}-\frac{r^{2}}{4 s} .
$$

Note that the choice of $\sigma^{*}=4 e^{3 / 2}$ in the denominator at the left hand side of $(5.28)$ is such that

$$
\lim _{N \rightarrow \infty} \frac{1}{N^{2}} \log \left[\frac{1}{(N / 2) !} Z_{N / 2,4 e^{3 / 2}}^{G U E}\right]=0,
$$

by (1.6), so that $F_{0}$ is the free energy defined by (1.8). The error term $\mathcal{O}\left(N^{-1}\right)$ in 5.28 can be expanded as an asymptotic series in negative integer powers of $N$ of the form

$$
\sum_{j=1}^{k} c^{(j)}(r, s) N^{-j}+\mathcal{O}\left(N^{-k-1}\right),
$$

for any $k \in \mathbb{N}$, where the coefficients $c^{(j)}(r, s)$ are analytic functions of $r$ and $s$.

For the computation of $\log Z_{2 N+1}$, the integrals in 3.50 do not cancel out exactly, but they contribute to the $\mathcal{O}(1)$-term. Substituting (5.23), 5.24) and (5.25), into (3.50), we obtain

$$
\begin{aligned}
\log Z_{2 N+1} & =\log (2 N+1) !+\log \left[\frac{1}{(N+1) !} Z_{N+1, s_{+}}^{G U E}\right]+\log \left[\frac{1}{N !} Z_{N, s_{-}}^{G U E}\right] \\
+ & \frac{(2 N+1)^{2} r^{2}}{4 s}-\frac{1}{2} \log \left((\sqrt{a}+\sqrt{b})(a b)^{1 / 4}\right)+\frac{1}{2} \log 2+\mathcal{O}\left(N^{-1}\right)
\end{aligned}
$$

as $N \rightarrow \infty$. Replacing $2 N+1$ by $N$, this becomes

$$
\begin{aligned}
\log Z_{N}=\log N & !+\log \left[\frac{1}{\left(\frac{N+1}{2}\right) !} Z_{(N+1) / 2, \widehat{s}_{+}}^{G U E}\right]+\log \left[\frac{1}{\left(\frac{N-1}{2}\right) !} Z_{(N-1) / 2, \widehat{s}_{-}}^{G U E}\right] \\
+ & \frac{N^{2} r^{2}}{4 s}-\frac{1}{2} \log \left((\sqrt{a}+\sqrt{b})(a b)^{1 / 4}\right)+\frac{1}{2} \log 2+\mathcal{O}\left(N^{-1}\right)
\end{aligned}
$$

for $N$ odd, where $\widehat{s}_{ \pm}=s\left(1 \pm \frac{1}{N}\right)$, and substracting $\log \left[\frac{Z_{(N-1) / 2,4 e^{3 / 2} Z_{(N+1) / 2,4 e^{3 / 2}}^{G U E}}^{G U E}}{\left(\frac{N-1}{2}\right) !\left(\frac{N+1}{2}\right) !}\right]$ on both sides, we find that

$$
\begin{array}{r}
\log \left[\frac{\left(\frac{N-1}{2}\right) !\left(\frac{N+1}{2}\right) ! Z_{N}}{N ! Z_{(N-1) / 2,4 e^{3 / 2}}^{G U E} Z_{(N+1) / 2,4 e^{3 / 2}}^{G U E}}\right]=\log \left[\frac{Z_{(N+1) / 2, \widehat{s}_{+}}^{G U E}}{Z_{(N+1) / 2,4 e^{3 / 2}}^{G U E}}\right]+\log \left[\frac{Z_{(N-1) / 2, \widehat{s}_{-}}^{G U E}}{Z_{(N-1) / 2,4 e^{3 / 2}}^{G U E}}\right] \\
+\frac{N^{2} r^{2}}{4 s}-\frac{1}{2} \log \left((\sqrt{a}+\sqrt{b})(a b)^{1 / 4}\right)+\frac{1}{2} \log 2+\mathcal{O}\left(N^{-1}\right) .
\end{array}
$$


By 1.5 and using the fact that $s=(b-a)^{2} / 4$, we finally obtain

$$
\begin{aligned}
\log \left[\frac{\left(\frac{N-1}{2}\right) !\left(\frac{N+1}{2}\right) ! Z_{N}}{N ! Z_{(N-1) / 2,4 e^{3 / 2}}^{G U E} Z_{(N+1) / 2,4 e^{3 / 2}}^{G U E}}\right] \\
\quad=-N^{2} F_{0}+\frac{1}{2} \log \left(\frac{\sqrt{b}-\sqrt{a}}{2(a b)^{1 / 4}}\right)+\mathcal{O}\left(N^{-1}\right) .
\end{aligned}
$$

The error term $\mathcal{O}\left(N^{-1}\right)$ in 5.33 can be expanded as an asymptotic series in negative integer powers of $N$ of the form

$$
\sum_{j=1}^{k} \widetilde{c}^{(j)}(r, s) N^{-j}+\mathcal{O}\left(N^{-k-1}\right)
$$

for any $k \in \mathbb{N}$, where the coefficients $\widetilde{c}^{(j)}(r, s)$ are analytic functions of $r$ and $s$. Theorem 2.2 is proven by 5.28 and 5.33 .

\section{Deformation of one-cut regular $V$ : proof of Theorem 2.8}

We assume in this section that $V=V_{\vec{t}} \in P_{m}^{(1)}$ is 1-cut regular. In general, the equilibrium measure associated to $V \in P_{m}^{(1)}$ can be written in the form [20]

$$
d \mu_{V}(x)=\frac{1}{i c} \mathcal{R}_{+}^{1 / 2}(x) h(x) d x, \quad x \in[a, b], \quad c>0
$$

where

$$
\mathcal{R}(z)=(z-a)(z-b)
$$

with $\mathcal{R}^{1 / 2}(z)$ analytic in $\mathbb{C} \backslash[a, b]$ and positive for $z>b$, and $\mathcal{R}_{+}^{1 / 2}(x), x \in(a, b)$, the boundary value from the upper half plane. The function $h$ is a monic polynomial of degree $\operatorname{deg} V-2$, and can be expressed as follows in terms of its zeros,

$$
h(z)=\prod_{j=1}^{n_{1}}\left(z-\gamma_{j}\right) . \prod_{j=1}^{n_{2}}\left(z-\eta_{j}\right) \cdot \prod_{j=1}^{n_{3}}\left(z-z_{j}\right)\left(z-\bar{z}_{j}\right),
$$

where

$$
\gamma_{n_{1}}<\ldots<\gamma_{2}<\gamma_{1}<a<b<\eta_{1}<\eta_{2}<\ldots<\eta_{n_{2}}, \operatorname{Im} z_{j} \geq 0, z_{j} \notin[a, b] .
$$

The number of zeros $n_{1}$ at the left of $[a, b]$ and the number of zeros $n_{2}$ at the right of $[a, b]$ are always even, the other zeros come in complex conjugate pairs. Using this notation, it is possible that some of the $z_{j}$ 's are real or coincide with each other or with one of the real zeros $\gamma_{j}, \eta_{j}$. Note that in the case of a quadratic external field $V$ as in Example 2.7, we have $n_{1}=n_{2}=n_{3}=0$.

A probability measure of the form (6.1) is not necessarily the equilibrium measure for an external field $V$, or in other words it does not necessarily belong to the image of the map $V \in P_{m}^{(1)} \mapsto \mu_{V} \in \mathcal{P}$. However, if a measure of the form 6.1 is an equilibrium 
measure, it follows from (2.17), after a straightforward calculation, that $V^{\prime}$ has to be equal to

$$
V^{\prime}(x)=\frac{2}{c} f_{a}^{b} \frac{\left|\mathcal{R}_{+}^{1 / 2}(y) h(y)\right|}{x-y} d y, \quad x \in[a, b],
$$

and consequently

$$
V^{\prime}(z)=\frac{1}{i c} \int_{\gamma} \frac{\mathcal{R}^{1 / 2}(y) h(y)}{z-y} d y
$$

with $\gamma$ a clock-wise oriented contour surrounding $[a, b]$ and $z$. The potential $V$ is uniquely fixed by the above condition and by $V(0)=0$.

Conversely, for any $\mu$ of the form (6.1), if we define $V^{\prime}$ as above, the variational equality (2.17) always holds. Nevertheless, it is still possible that the variational inequality (2.18) is violated, and in that case $\mu$ is not an equilibrium measure. The following lemma gives a convenient characterization of the image of the map $V \in$ $P_{m}^{(1)} \mapsto \mu_{V} \in \mathcal{P}$.

Define

$$
\begin{array}{ll}
m_{j}=\int_{\eta_{j}}^{\eta_{j+1}}\left|\mathcal{R}^{1 / 2}(x) h(x)\right| d x \geq 0, & j=0,1, \ldots, n_{2}-1, \\
\tilde{m}_{j}=\int_{\gamma_{j+1}}^{\gamma_{j}}\left|\mathcal{R}^{1 / 2}(x) h(x)\right| d x \geq 0, & j=0,1, \ldots, n_{1}-1,
\end{array}
$$

where we use the convention that $\gamma_{0}=a, \eta_{0}=b$.

Lemma 6.1 Let $h$ be of the form (6.3)- (6.4) with $n_{1}, n_{2}$ even, and let $\mu$ be defined by

$$
d \mu(x)=\frac{1}{c}\left|\mathcal{R}^{1 / 2}(x) h(x)\right| d x, \quad x \in[a, b], \quad c=\int_{a}^{b}\left|\mathcal{R}^{1 / 2}(x) h(x)\right| d x .
$$

Let $V$ be given by (6.6) with integration constant fixed by the condition $V(0)=0$. Then, $V$ is one-cut regular $\left(V \in P_{m}^{(1)}\right)$ and $\mu$ is equal to the equilibrium measure $\mu_{V}$ if and only if

$$
\begin{array}{ll}
\sum_{j=0}^{k-1}(-1)^{j} m_{j}>0, & 0<k \leq n_{2}, k \text { even }, \\
\sum_{j=0}^{k-1}(-1)^{j} \tilde{m}_{j}>0, & 0<k \leq n_{1}, k \text { even } .
\end{array}
$$

Proof. Let us consider the function

$$
G(z)=\frac{1}{\pi i} \int_{a}^{b} \frac{d \mu(y)}{z-y}=-\frac{1}{\pi c} \int_{a}^{b} \frac{\mathcal{R}_{+}^{1 / 2}(y) h(y)}{z-y} d y .
$$

By Cauchy's theorem for $z \notin[a, b]$,

$$
G(z)=-\frac{1}{i c} \mathcal{R}^{1 / 2}(z) h(z)-\frac{1}{2 \pi c} \int_{\gamma} \frac{\mathcal{R}^{1 / 2}(y) h(y)}{z-y} d y
$$


where $\gamma$ is a clock-wise contour surrounding the interval $[a, b]$ and $z$. By $(6.6)$, we have

$$
G(z)=-\frac{1}{i c} \mathcal{R}^{1 / 2}(z) h(z)+\frac{1}{2 \pi i} V^{\prime}(z), \quad z \notin[a, b] .
$$

It follows that

$$
G_{+}(x)+G_{-}(x)=\frac{1}{\pi i} V^{\prime}(x), \quad x \in[a, b],
$$

and upon integrating, we have that there exists a constant $\ell_{V}$ such that

$$
2 \int_{a}^{b} \log |x-y| d \mu(y)-V(x)=\ell_{V}, \quad x \in[a, b] .
$$

Evaluating at $x=b$ and $x=a$, we obtain

$$
\ell_{V}=2 \int \log |b-y| d \mu(y)-V(b)=2 \int \log |a-y| d \mu(y)-V(a) .
$$

Recall that the equilibrium measure $\mu_{V}$ is characterized by the variational equality (2.17) and the variational inequality (2.18). The measure $\mu$ already satisfies the equality by (6.14), and this means that $\mu$ is equal to the equilibrium measure $\mu_{V}$ if and only if

$$
2 \int_{a}^{b} \log |x-y| d \mu(y)-V(x) \leq \ell_{V}, \quad x \in \mathbb{R} .
$$

Moreover, since $h$ has no zeros on $[a, b], V$ is one-cut regular and $\mu=\mu_{V}$ if and only if

$$
2 \int_{a}^{b} \log |x-y| d \mu(y)-V(x)<\ell_{V}, \quad x \in \mathbb{R} \backslash[a, b],
$$

which is by 6.15) equivalent to

$$
2 \int_{a}^{b} \log \left|\frac{x-y}{b-y}\right| d \mu(y)-\int_{b}^{x} V^{\prime}(\xi) d \xi<0, \quad x \in \mathbb{R} \backslash[a, b] .
$$

For $x>b$, by 6.12 , this inequality can be written as

$$
\int_{b}^{x}\left[2 \pi i G(\xi)-V^{\prime}(\xi)\right] d \xi<0
$$

or, by 6.13),

$$
H_{b}(x):=\frac{1}{c} \int_{b}^{x} \mathcal{R}^{1 / 2}(\xi) h(\xi) d \xi>0, \quad x>b .
$$

Repeating the same computation for $x<a$, we get

$$
H_{a}(x):=\frac{1}{c} \int_{x}^{a} \mathcal{R}^{1 / 2}(\xi) h(\xi) d \xi>0, \quad x<a .
$$

(i) Assume first that $\mu=\mu_{V}, V \in P_{m}^{(1)}$. Then we have 6.17, which implies 6.18 and 6.19 by the above discussion, and in particular we have

$$
\begin{aligned}
& 0<H_{b}\left(\eta_{k}\right)=\frac{1}{c} \int_{b}^{\eta_{k}} \mathcal{R}^{1 / 2}(\xi) h(\xi) d \xi=\frac{1}{c}\left(m_{0}-m_{1}+\ldots-m_{k-1}\right), \\
& 0<H_{a}\left(\gamma_{k}\right)=\frac{1}{c} \int_{\gamma_{k}}^{a} \mathcal{R}^{1 / 2}(\xi) h(\xi) d \xi=\frac{1}{c}\left(\tilde{m}_{0}-\tilde{m}_{1}+\ldots-\tilde{m}_{k-1}\right),
\end{aligned}
$$

for $k>0$ even. This implies 6.10-6.11. 
(ii) Conversely, assume that $6.10-(6.11)$ hold. In order to show that $\mu$ is the equilibrium measure, we need to prove (6.18) and (6.19). From the fact that $h$ is positive on $[a, b]$ and changes sign at $\eta_{1}, \ldots, \eta_{n_{2}}, \gamma_{1}, \ldots, \gamma_{n_{1}}$, it is clear that $H_{b}(x)$ is increasing for $\eta_{2 j}<x<\eta_{2 j+1}$ and decreasing for $\eta_{2 j+1}<x<\eta_{2 j+2}$, while $H_{a}(x)$ is decreasing for $\gamma_{2 j+1}<x<\gamma_{2 j}$, and increasing for $\gamma_{2 j+2}<x<\gamma_{2 j+1}$. Consequently, it is sufficient to prove that $H_{a}\left(\gamma_{k}\right)>0$ and $H_{b}\left(\eta_{k}\right)>0$ for $k>0$ even. This follows from the equalities in 6.20)-6.21).

We will prove Theorem 2.8 by induction on $n_{1}+n_{2}$. i.e. on the number of real zeros of $h$ with odd multiplicity.

\subsection{Basis $n_{1}+n_{2}=0$}

We assume here that $V=V_{\vec{t}} \in P_{m}^{(1)}$, and that $\mu$ is of the form 6.1)- 6.3 with $n_{1}+n_{2}=$ 0 . We will now define a continuous deformation $V=V_{\vec{t}(s)}, s \in[0,1]$, of $V$ such that $V_{\vec{t}(s)} \in P_{m}^{(1)}$ and such that the following three conditions hold:

(i) $V_{\vec{t}(1)}(x)=V_{\vec{t}}(x)$,

(ii) $V_{\vec{t}(0)}(x)=\frac{x^{2}}{2}$,

(iii) for all $s \in[0,1], V_{\vec{t}(s)} \in P_{m}^{(1)}$.

We will define this deformation implicitly by deforming the equilibrium measure $\mu_{V}$ to a measure $\mu_{s}$ for $s \in[0,1]$ such that

$$
\mu_{1}=\mu_{V}, \quad \mu_{0}=\mu_{x^{2} / 2} .
$$

We will then use Lemma 6.1 to prove that $\mu_{s}$ is an equilibrium measure for all $s \in(0,1]$. The deformation of $\mu$ defines a deformation of $V$ by (6.6).

If $n_{1}+n_{2}=0, h$ has no real zeros of odd multiplicity and can be written as

$$
h(z)=\prod_{j=1}^{n_{3}}\left(z-z_{j}\right)\left(z-\bar{z}_{j}\right) .
$$

Let us define, for $s \in(0,1]$,

$$
\begin{array}{ll}
h(z ; s)=\prod_{j=1}^{n_{3}}\left(z-z_{j}(s)\right)\left(z-\bar{z}_{j}(s)\right), & z_{j}(s)=z_{j}+i \frac{1-s}{s}, \\
\mathcal{R}(z ; s)=(z-a(s))(z-b(s)), & b(s)=b s+2(1-s), \\
a(s)=s a-2(1-s), &
\end{array}
$$

and

$$
d \mu_{s}(x)=\frac{1}{i c(s)} \mathcal{R}_{+}^{1 / 2}(x ; s) h(x ; s) d x, \quad x \in[a(s), b(s)],
$$

where

$$
c(s)=-i \int_{a(s)}^{b(s)} \mathcal{R}_{+}^{1 / 2}(x ; s) h(x ; s) d x
$$


is chosen such that $\mu_{s}$ has total mass 1 .

For all $s \in(0,1), h$ has no sign changes on the real line and thus satisfies automatically the conditions of Lemma 6.1. We have that $\mu_{s}$ is the equilibrium measure corresponding to the external field $\sqrt{6.6}$ for all $s \in(0,1)$, given by

$$
V_{\vec{t}(s)}^{\prime}(z)=\frac{1}{i c(s)} \int_{\gamma} \frac{\mathcal{R}^{1 / 2}(y ; s) h(y ; s)}{z-y} d y .
$$

Since the dependence of $V$ on the coefficients of $h$ and $\mathcal{R}$ is continuous, $\vec{t}(s)$ describes a continuous path for $0<s \leq 1$. Moreover, it is straightforward to verify by residue calculus that $V_{\vec{t}(s)}$ is a polynomial of degree $\operatorname{deg} h+2=\operatorname{deg} V$ for $0<s<1$.

When taking the limit $s \rightarrow 0$, the zeros of $h$ tend to infinity and $c(s) \rightarrow \infty$, and one verifies that $\frac{1}{c(s)} h(z ; s) \rightarrow \frac{1}{2 \pi}$ uniformly on compacts. Indeed, as $s \rightarrow \infty$, we have

$$
\begin{aligned}
\frac{1}{c(s)} h(z ; s) & =\frac{\prod_{j=1}^{n_{3}}\left(z-z_{j}(s)\right)\left(z-\bar{z}_{j}(s)\right)}{\int_{a(s)}^{b(s)}\left|\mathcal{R}_{+}^{1 / 2}(x ; s)\right| h(x ; s) d x} \\
& \sim \frac{s^{-2 n_{3}}}{s^{-2 n_{3}} \int_{-2}^{2} \sqrt{4-x^{2}} d x}=\frac{1}{2 \pi} .
\end{aligned}
$$

We thus obtain

$$
d \mu_{0}(x)=\frac{1}{2 \pi} \sqrt{4-x^{2}} d x, \quad x \in[-2,2],
$$

and

$$
V_{\vec{t}(0)}(z)=\frac{1}{2 \pi i} \int_{\gamma} \frac{[(y-2)(y+2)]^{1 / 2}}{z-y} d y=z^{2} / 2 .
$$

This completes the construction of the deformation of $V$ in the case $n_{1}+n_{2}=0$.

\subsection{Inductive step}

We assume that Theorem 2.8 holds true for any external field $\tilde{V} \in P_{m}^{(1)}$ which is such that $\tilde{h}$ in 6.9 is of the form 6.3)- 6.4 with $n_{1}+n_{2}<N_{1}+N_{2}$, i.e. $\tilde{h}$ has strictly less than $N_{1}+N_{2}$ real zeros of odd multiplicity, with $N_{1}+N_{2}>0$. Next, let us consider $h$ of the form

$$
h(z)=\prod_{j=1}^{N_{1}}\left(z-\gamma_{j}\right) \cdot \prod_{j=1}^{N_{2}}\left(z-\eta_{j}\right) \cdot \prod_{j=1}^{N_{3}}\left(z-z_{j}\right)\left(z-\bar{z}_{j}\right), \quad \operatorname{Im} z_{j} \geq 0, z_{j} \notin[a, b],
$$

where

$$
\gamma_{N_{1}}<\ldots<\gamma_{2}<\gamma_{1}<\gamma_{0}=a<b=\eta_{0}<\eta_{1}<\eta_{2}<\ldots<\eta_{N_{2}} .
$$

At least one of the integers $N_{1}, N_{2}$ is stictly positive; we will assume $N_{2}>0$ here, if $N_{2}=0$, the case $N_{1}>0$ can be handled similarly. We will construct a path $V_{\vec{t}(s)}$ which connects $V_{\vec{t}(1)}$ to an external field $\tilde{V}=V_{\vec{t}(0)}$ which satisfies the condition $n_{1}+n_{2}<N_{1}+N_{2}$. The composition of this path with the path connecting $V_{\vec{t}(0)}$ to the Gaussian $z^{2} / 2$ (which exists because of the induction hypothesis), completes the construction of the path required for the proof of Theorem 2.8. 
Let us deform $h(z)$ to $h(z ; s)$ for $s \in[0,1]$ in such a way that $h(z ; 1)=h(z)$, and $h(z ; 0)$ has only $N_{1}+N_{2}-2$ real zeros of odd multiplicity. Define

$$
h(z ; s)=\left(z-\eta_{1}(s)\right) \prod_{j=1}^{N_{1}}\left(z-\gamma_{j}\right) . \prod_{j=2}^{N_{2}}\left(z-\eta_{j}\right) \prod_{j=1}^{N_{3}}\left(z-z_{j}\right)\left(z-\bar{z}_{j}\right),
$$

where we deform only one zero $\eta_{1}$ as follows:

$$
\eta_{1}(s)=s \eta_{1}+(1-s) \eta_{2} .
$$

We then clearly have $h(z ; 1)=h(z)$, and $h(z ; 0)$ has only $N_{1}+N_{2}-2$ zeros of odd multiplicity, since $\eta_{1}$ merged with $\eta_{2}$, which has now become a zero of even multiplicity. Let us consider the measure

$$
d \mu_{s}(x)=\frac{1}{i c(s)} \mathcal{R}_{+}^{1 / 2}(x) h(x ; s) d x, \quad x \in[a, b],
$$

where $c(s)$ is chosen such that $\mu_{s}$ is a probability measure. The deformation of $\mu$ induces as before a deformation of $V$ to $V_{\vec{t}(s)}$ by $(6.6)$, which preserves the degree of $V$ since the degree of $h$ is unchanged. What remains to prove, is that $\mu_{s}$ is equal to the equilibrium measure in external field $V_{\vec{t}(s)}$. To that end, by Lemma 6.1, we need to prove only that 6.10-6.11 hold for $0<s<1$.

Recall the definition (6.7)- 6.8 of $m_{j}$ and $\tilde{m}_{j}$, which now depend on $s$. We have

$$
\begin{aligned}
& m_{j}(s)=\int_{\eta_{j}}^{\eta_{j+1}}\left|\mathcal{R}^{1 / 2}(x) h(x ; s)\right| d x=\int_{\eta_{j}}^{\eta_{j+1}}\left|\mathcal{R}^{1 / 2}(x) h(x) f(x ; s)\right| d x \\
& \tilde{m}_{j}(s)=\int_{\gamma_{j+1}}^{\gamma_{j}}\left|\mathcal{R}^{1 / 2}(x) h(x) f(x ; s)\right| d x
\end{aligned}
$$

where

$$
f(x ; s)=\frac{x-\eta_{1}(s)}{x-\eta_{1}} .
$$

Note that $f(x ; s)$ is positive, increasing, and smaller than 1 for $x>\eta_{1}(s)$, and that $f(x ; s)$ is bigger than 1 and increasing for $x<\eta_{1}$. As a consequence, we have the inequalities $m_{0}(s) \geq m_{0}$ and

$$
\begin{aligned}
& m_{j}(s) \geq m_{j} f\left(\eta_{j} ; s\right), \quad m_{j}(s) \leq m_{j} f\left(\eta_{j+1} ; s\right), \quad 1 \leq j \leq N_{2}, \\
& \tilde{m}_{j}(s) \geq \tilde{m}_{j} f\left(\gamma_{j+1} ; s\right), \quad \tilde{m}_{j}(s) \leq \tilde{m}_{j} f\left(\gamma_{j} ; s\right), \quad 0 \leq j \leq N_{1} .
\end{aligned}
$$

This implies that

$$
\begin{aligned}
m_{2 j-1}(s)-m_{2 j}(s) \leq f\left(\eta_{2 j} ; s\right) & \left(m_{2 j-1}-m_{2 j}\right) \\
& <\left(m_{2 j-1}-m_{2 j}\right), \quad j=1, \ldots, \frac{n_{2}}{2}-1,
\end{aligned}
$$

and $m_{k-1}(s) \leq m_{k-1}$ for $k>0$ even. We obtain

$$
\begin{aligned}
& m_{0}(s)-m_{1}(s)+m_{2}(s)-\ldots-m_{k-1}(s) \\
& \quad \geq m_{0}-\left(m_{1}-m_{2}\right)-\left(m_{3}-m_{4}\right)-\ldots-\left(m_{k-3}-m_{k-2}\right)-m_{k-1} \\
& \quad=\sum_{j=0}^{k-1}(-1)^{j} m_{j}>0,
\end{aligned}
$$


by Lemma 6.1. Similarly,

$$
\begin{aligned}
\tilde{m}_{0}(s) & -\tilde{m}_{1}(s)+\tilde{m}_{2}(s)-\ldots-\tilde{m}_{\ell}(s) \\
& \geq f\left(\gamma_{1} ; s\right)\left(\tilde{m}_{0}-\tilde{m}_{1}\right)+f\left(\gamma_{3} ; s\right)\left(\tilde{m}_{2}-\tilde{m}_{3}\right)+\ldots+f\left(\gamma_{\ell} ; s\right)\left(\tilde{m}_{\ell-1}-\tilde{m}_{\ell}\right) \\
& \geq\left(\tilde{m}_{0}-\tilde{m}_{1}\right)+\left(\tilde{m}_{2}-\tilde{m}_{3}\right)+\ldots+\left(\tilde{m}_{\ell-1}-\tilde{m}_{\ell}\right) \\
& =\sum_{j=0}^{\ell}(-1)^{j} \tilde{m}_{j}>0 .
\end{aligned}
$$

This proves 6.10) -6.11) for $0<s<1$ and completes the proof of Theorem 2.8.

\section{Deformation of two-cut regular $V$ : proof of Theorem 2.9}

We proceed in a similar way as for the proof in the one-cut case. Assume now that $V=V_{\vec{t}} \in P_{m}^{(2)}$ is 2-cut regular. The equilibrium measure then has the form [20]

$$
d \mu_{V}(x)=\frac{1}{i c} \mathcal{R}_{+}^{1 / 2}(x) h(x) d x, \quad x \in\left[a_{1}, a_{2}\right] \cup\left[a_{3}, a_{4}\right],
$$

with

$$
\mathcal{R}(z)=\left(z-a_{1}\right)\left(z-a_{2}\right)\left(z-a_{3}\right)\left(z-a_{4}\right), \quad a_{1}<a_{2}<a_{3}<a_{4},
$$

where $\mathcal{R}^{1 / 2}(z)$ analytic in $\mathbb{C} \backslash\left(\left[a_{1}, a_{2}\right] \cup\left[a_{3}, a_{4}\right]\right)$ and positive for $z>a_{4}$. The function

$h$ is a monic polynomial of degree $\operatorname{deg} V-3$, which can be written in terms of its zeros as follows,

$$
h(z)=\prod_{j=1}^{n_{1}}\left(z-\gamma_{j}\right) \cdot \prod_{j=1}^{n_{2}}\left(z-\xi_{j}\right) \cdot \prod_{j=1}^{n_{3}}\left(z-\eta_{j}\right) \cdot \prod_{j=1}^{n_{4}}\left(z-z_{j}\right)\left(z-\bar{z}_{j}\right),
$$

with $\operatorname{Im} z_{j} \geq 0, z_{j} \notin\left[a_{1}, b_{1}\right] \cup\left[a_{2}, b_{2}\right]$ and

$$
\begin{array}{r}
\gamma_{n_{1}}<\ldots<\gamma_{2}<\gamma_{1}<\gamma_{0}=a_{1}<a_{2}=\xi_{0}<\xi_{1}<\ldots<\xi_{n_{2}}<\xi_{n_{2}+1}=a_{3} \\
<a_{4}=\eta_{0}<\eta_{1}<\eta_{2}<\ldots<\eta_{n_{3}},
\end{array}
$$

with $n_{1}, n_{3}$ even, $n_{2}$ odd. In other words, the $\gamma_{j}$ 's are the real zeros of odd multiplicity at the left of $a_{1}$, the $\xi_{j}$ 's are the zeros of odd multiplicity in the gap $\left(a_{2}, a_{3}\right)$, and the $\eta_{j}$ 's are the real zeros of odd multiplicity at the right of $a_{4}$. Again, it is possible that some of the $z_{j}$ 's are real and coincide with each other or with one of the real zeros $\gamma_{j}, \xi_{j}, \eta_{j}$. Define

$$
\begin{array}{lr}
m_{j}=\int_{\eta_{j}}^{\eta_{j+1}}\left|\mathcal{R}^{1 / 2}(x) h(x)\right| d x, & j=0, \ldots, n_{3}-1 ; \\
\tilde{m}_{j}=\int_{\gamma_{j+1}}^{\gamma_{j}}\left|\mathcal{R}^{1 / 2}(x) h(x)\right| d x, & j=0, \ldots, n_{1}-1, \\
\widehat{m}_{j}=\int_{\xi_{j}}^{\xi_{j+1}}\left|\mathcal{R}^{1 / 2}(x) h(x)\right| d x, & j=0, \ldots, n_{2} .
\end{array}
$$


Lemma 7.1 Let $h$ be of the form (7.3)-(7.4) with $n_{1}, n_{3}$ even and $n_{2}$ odd, and let $\mu$ be defined by

$$
\begin{aligned}
& d \mu(x)=\frac{1}{i c} \mathcal{R}_{+}^{1 / 2}(x) h(x) d x, \quad x \in\left[a_{1}, a_{2}\right] \cup\left[a_{3}, a_{4}\right], \\
& c=\int_{J}\left|\mathcal{R}(x)^{1 / 2} h(x)\right| d x, \quad J=\left[a_{1}, a_{2}\right] \cup\left[a_{3}, a_{4}\right] .
\end{aligned}
$$

Let $V$ be given by

$$
V^{\prime}(z)=\frac{1}{i c} \int_{\gamma} \frac{\mathcal{R}^{1 / 2}(y) h(y)}{z-y} d y, \quad z \in \mathbb{C} \backslash J,
$$

with $\gamma$ a clock-wise oriented contour surrounding $\left[a_{1}, a_{2}\right] \cup\left[a_{3}, a_{4}\right]$ and $z$. The constant of integration is fixed by $V(0)=0$. Then, $V$ is two-cut regular $\left(V \in P_{m}^{(2)}\right)$ and $\mu$ is equal to the equilibrium measure $\mu_{V}$ if and only if

$$
\begin{array}{lr}
\sum_{j=0}^{k-1}(-1)^{j} m_{j}>0, & k=2,4,6, \ldots, n_{1}, \\
\sum_{j=0}^{k-1}(-1)^{j} \tilde{m}_{j}>0, & k=2,4,6, \ldots, n_{3}, \\
\sum_{j=0}^{k-1}(-1)^{j} \widehat{m}_{j}>0, & k=2,4,6, \ldots, n_{2}-1, \\
\sum_{j=0}^{n_{2}}(-1)^{j} \widehat{m}_{j}=0 . &
\end{array}
$$

Proof. Let $\mu$ be of the form (7.8), and $V$ as in $(7.9)$. In a similar way as in the proof of Lemma 6.1, one can show that the variational conditions (2.17)- 2.18) are equivalent to (see [21])

$$
\begin{aligned}
H_{4}(x) & :=\frac{1}{c} \int_{a_{4}}^{x} \mathcal{R}^{1 / 2}(\xi) h(\xi) d \xi>0, & & x>a_{4}, \\
H_{2}(x) & :=\frac{1}{c} \int_{a_{2}}^{x} \mathcal{R}^{1 / 2}(\xi) h(\xi) d \xi<0, & & a_{2}<x<a_{3}, \\
H_{2}\left(a_{3}\right) & =0, & & \\
H_{1}(x) & :=\frac{1}{c} \int_{x}^{a_{1}} \mathcal{R}^{1 / 2}(\xi) h(\xi) d \xi>0, & & x<a_{1} .
\end{aligned}
$$

(i) Assume that $\mu=\mu_{V}, V \in P_{m}^{(2)}$. Then by 7.14-7.17 we have

$$
\begin{aligned}
0 & <\frac{1}{c} \int_{a_{4}}^{\eta_{j}} \mathcal{R}^{1 / 2}(\xi) h(\xi) d \xi=\frac{1}{c}\left(m_{0}-m_{1}+\ldots-m_{j}\right), \\
0 & >\frac{1}{c} \int_{a_{2}}^{\xi_{j}} \mathcal{R}^{1 / 2}(\xi) h(\xi) d \xi=-\frac{1}{c}\left(\widehat{m}_{0}-\widehat{m}_{1}+\ldots-\widehat{m}_{j}\right), \\
0 & <\frac{1}{c} \int_{\gamma_{j}}^{a_{1}} \mathcal{R}^{1 / 2}(\xi) h(\xi) d \xi=\frac{1}{c}\left(\tilde{m}_{0}-\tilde{m}_{1}+\ldots-\tilde{m}_{j}\right) .
\end{aligned}
$$

This implies 7.10-7.12). By 7.16, we also have 7.13. 
(ii) Conversely, assume that 7.10)-(7.13) hold. We need to prove the strict inequality (2.18) for $x \in \mathbb{R} \backslash\left(\left[a_{1}, a_{2}\right] \cup\left[a_{3}, a_{4}\right]\right)$ which is equivalent to $H_{4}(x)>0$ for $x>a_{4}$, $H_{2}(x)<0$ for $x \in\left(a_{2}, a_{3}\right)$ and $H_{1}(x)>0$ for $x<a_{1}$. From 7.18 we know that $H_{4}(x)$ is increasing for $\eta_{2 j}<x<\eta_{2 j+1}$ and decreasing for $\eta_{2 j+1}<x<\eta_{2 j+2}$. Since $H_{4}\left(\eta_{j}\right)>0$ for all $j=1, \ldots, n_{1}$ it follows that $H_{4}(x)$ is strictly positive for $x>a_{4}$. The same reasoning can be applied to $H_{1}(x)$ and $H_{2}(x)$. In order to prove the variational equality (2.17) we observe that integrating the second equality in (7.9) between $a_{1}$ and $x \in\left[a_{1}, a_{2}\right]$ we have

$$
V(x)-V\left(a_{1}\right)=2 \int_{J} \log |x-s| \psi(s) d s-2 \int_{J} \log \left|a_{1}-s\right| \psi(s) d s,
$$

which shows that $V(x)-2 \int_{J} \log |x-s| \psi(s) d s$ is constant on $\left[a_{1}, a_{2}\right]$. In the same way we can show that $V(x)-2 \int_{J} \log |x-s| \psi(s) d s$ is constant on $\left[a_{3}, a_{4}\right]$. The condition (7.13) guarantees that the constant is the same on the two intervals.

We will prove Theorem 2.9 by induction on $n_{1}+n_{2}+n_{3}$. Since $n_{2}$ is odd, we have $n_{1}+n_{2}+n_{3} \geq 1$.

\subsection{Basis $n_{1}+n_{2}+n_{3}=1$}

If $n_{1}+n_{2}+n_{3}=1, h$ has only one real odd multiplicity zero $\xi$, which has to lie in $\left(a_{2}, a_{3}\right)$ since $n_{2}$ is odd and $n_{1}, n_{3}$ are even. We have

$$
h(z)=(z-\xi) \prod_{j=1}^{n_{4}}\left(z-z_{j}\right)\left(z-\bar{z}_{j}\right) .
$$

As before, we will deform the equilibrium measure $\mu$ to the measure 2.2 in the case $s=1, r=4$, which will induce a deformation of $V(z)$ to the quartic potential $z^{4}-4 z^{2}$. Similarly as in the one-cut case, we do this by sending all zeros $z_{j}$ of $h$ to $\infty$, but as opposed to the one-cut case, where we were free to deform the zeros, here we need to make sure that the deformation preserves the constraint $\widehat{m}_{0}-\widehat{m}_{1}=0$. Otherwise, the deformed measure $\mu$ will not be an equilibrium measure.

For $s \in[0,1]$, we define

$$
\mathcal{R}(z ; s)=\left(z-a_{1}(s)\right)\left(z-a_{2}(s)\right)\left(z-a_{3}(s)\right)\left(z-a_{4}(s)\right),
$$

with

$$
\begin{array}{lr}
a_{1}(s)=s a_{1}-\sqrt{3}(1-s), & a_{2}(s)=s a_{2}-(1-s), \\
a_{3}(s)=s a_{3}+(1-s), & a_{4}(s)=s a_{4}+\sqrt{3}(1-s),
\end{array}
$$

and

$$
h(z ; s)=(z-\xi(s)) \prod_{j=1}^{n_{3}}\left(z-z_{j}(s)\right)\left(z-\bar{z}_{j}(s)\right), \quad z_{j}(s)=z_{j}+i \frac{1-s}{s},
$$

where $\xi(s) \in\left(a_{2}, a_{3}\right)$ is chosen in such a way that $\widehat{m}_{0}-\widehat{m}_{1}=0$. Note that there is a unique value of $\xi(s)$ such that this is true, and that it depends continuously on $s$. We let

$$
d \mu_{s}(x)=\frac{1}{i c(s)} \mathcal{R}_{+}^{1 / 2}(x ; s) h(x ; s) d x, \quad x \in\left[a_{1}, a_{2}\right] \cup\left[a_{3}, a_{4}\right],
$$


where $c(s)$ is chosen such that $\mu_{s}$ has mass 1 .

For all $s \in(0,1), h$ has only one real zero and thus satisfies automatically the conditions $(7.10)-(7.12)$. The fourth condition $(7.13)$ holds by definition of $\xi(s)$. When taking the limit $s \rightarrow 0$, the non-real zeros $z_{j}(s)$ tend to infinity, and $d \mu_{s}$ converges to

$$
d \mu_{0}(x)=\frac{2}{\pi}|x| \sqrt{\left(3-x^{2}\right)\left(x^{2}-1\right)} d x, \quad x \in[-\sqrt{3},-1] \cup[1, \sqrt{3}] .
$$

By $(7.9)$, we have that $\mu_{s}$ is the equilibrium measure corresponding to external field

$$
V_{\vec{t}(s)}^{\prime}(z)=\frac{1}{i c(s)} \int_{\gamma} \frac{\mathcal{R}^{1 / 2}(y, s) h(y, s)}{z-y} d y, \quad z \in \mathbb{C} \backslash J(s),
$$

for all $s \in[0,1]$. Since the dependence of $V$ in $\sqrt{7.9}$ on the coefficients of $h$ and $\mathcal{R}$ is continuous, $\vec{t}(s)$ describes a continuous path. In addition, we have $V_{\vec{t}(0)}(z)=z^{4}-4 z^{2}$, see (2.1)-(2.2), and the degree of $V_{\vec{t}(s)}$ is the same as the degree of $V$ for all $0<s<1$.

\section{$7.2 \quad$ Inductive step}

We now suppose that Theorem 2.8 holds for any $h$ of the form (6.3)- 6.4 with $n_{1}+$ $n_{2}+n_{3}<N_{1}+N_{2}+N_{3}$, i.e. if $h$ has strictly less than $N_{1}+N_{2}+N_{3}$ real zeros of odd multiplicity, with $N_{1}+N_{2}+N_{3}>1$. Let $V=V_{\vec{t}(1)} \in P_{m}^{(1)}$ be such that $h$ has $N_{1}+N_{2}+N_{3}$ real zeros of odd multiplicity. Our strategy is as before to deform $V_{\vec{t}(1)}$ by means of a deformation of $\mu$, to an external field $V_{\vec{t}\left(s_{0}\right)}$ for which $h$ has less than $N_{1}+N_{2}+N_{3}$ zeros. Afterwards, in order to complete the proof, the path $V_{\vec{t}(s)}$, $s \in\left[s_{0}, 1\right]$, has to be composed with the path connecting $V_{\vec{t}\left(s_{0}\right)}$ to the quartic external field $V_{\vec{t}(0)}=z^{4}-4 z^{2}$. The latter exists because of the induction hypothesis. There are two cases which we will treat separately: $N_{2}>1$ and $N_{2}=1$.

Case 1: $N_{2}>1$

First consider the case where $N_{2}>1$. Define a deformation of $h$ as follows:

$$
h(z)=\left(z-\xi_{1}(s)\right)\left(z-\xi_{N_{2}}(s)\right) \prod_{j=1}^{N_{1}}\left(z-\gamma_{j}\right) . \prod_{j=2}^{N_{2}-1}\left(z-\xi_{j}\right) \cdot \prod_{j=1}^{N_{3}}\left(z-\eta_{j}\right) \cdot \prod_{j=1}^{N_{4}}\left(z-z_{j}\right)\left(z-\bar{z}_{j}\right),
$$

where

$$
\xi_{1}(s)=\xi_{1}+(1-s),
$$

and where we choose $\xi_{N_{2}}(s)$ in such a way that $(7.13)$ holds.

One verifies that $\xi_{N_{2}}(s)$ is uniquely defined and smooth for $s$ close to 1 . We claim that in addition $\xi_{N_{2}}(s)$ is increasing in $s$.

Proof of the claim: By definition of $\xi_{N_{2}}(s)$, we have $\sum_{j=0}^{n_{2}}(-1)^{j} \widehat{m}_{j}(s)=0$. Since the $m_{j}$ 's depend on $\xi_{1}, \xi_{N_{2}}$ by (7.7), we have

$$
\begin{aligned}
0 & =\frac{\partial}{\partial s} \sum_{j=0}^{n_{2}}(-1)^{j} \widehat{m}_{j}(s) \\
& =\frac{\partial \xi_{1}(s)}{\partial s} \frac{\partial}{\partial \xi_{1}} \sum_{j=0}^{n_{2}}(-1)^{j} \widehat{m}_{j}\left(\xi_{1}, \xi_{N_{2}}\right)+\frac{\partial \xi_{N_{2}}(s)}{\partial s} \frac{\partial}{\partial \xi_{N_{2}}} \sum_{j=0}^{n_{2}}(-1)^{j} \widehat{m}_{j}\left(\xi_{1}, \xi_{N_{2}}\right)
\end{aligned}
$$


We write $\widehat{m}_{j}\left(\xi_{1}, \xi_{N_{2}}\right)$ here since all the other $\xi_{j}$ 's are constant. If we prove that

$$
\begin{aligned}
& \frac{\partial}{\partial \xi_{1}} \sum_{j=0}^{n_{2}}(-1)^{j} \widehat{m}_{j}\left(\xi_{1}, \xi_{N_{2}}\right)>0 \\
& \frac{\partial}{\partial \xi_{N_{2}}} \sum_{j=0}^{n_{2}}(-1)^{j} \widehat{m}_{j}\left(\xi_{1}, \xi_{N_{2}}\right)>0
\end{aligned}
$$

it follows from $\frac{\partial \xi_{1}(s)}{\partial s}<0$ that $\frac{\partial \xi_{N_{2}}(s)}{\partial s}>0$.

For $\xi_{1}^{\prime}>\xi_{1}$, we have

$$
\widehat{m}_{j}\left(\xi_{1}^{\prime}, \xi_{N_{2}}\right)=\int_{\xi_{j}}^{\xi_{j+1}}\left|\mathcal{R}^{1 / 2}(x) h(x) F\left(x ; \xi_{1}^{\prime}, \xi_{1}\right)\right| d x,
$$

with

$$
F\left(x ; \xi_{1}^{\prime}, \xi_{1}\right)=\frac{x-\xi_{1}^{\prime}}{x-\xi_{1}}
$$

It follows immediately that

$$
\widehat{m}_{0}\left(\xi_{1}^{\prime}, \xi_{N_{2}}\right)>\widehat{m}_{0}\left(\xi_{1}, \xi_{N_{2}}\right), \quad \widehat{m}_{n_{2}}\left(\xi_{1}^{\prime}, \xi_{N_{2}}\right)<\widehat{m}_{n_{2}}\left(\xi_{1}, \xi_{N_{2}}\right),
$$

and, since $F$ is increasing and smaller than 1 for $x>\xi_{1}$, we also have

$$
\begin{aligned}
\widehat{m}_{2 j-1}\left(\xi_{1}^{\prime}, \xi_{N_{2}}\right)-\widehat{m}_{2 j}\left(\xi_{1}^{\prime}, \xi_{N_{2}}\right) \leq F\left(\xi_{2 j}\right) & \left(\widehat{m}_{2 j-1}\left(\xi_{1}, \xi_{N_{2}}\right)-\widehat{m}_{2 j}\left(\xi_{1}, \xi_{N_{2}}\right)\right) \\
& \left.<\widehat{m}_{2 j-1}\left(\xi_{1}, \xi_{N_{2}}\right)-\widehat{m}_{2 j}\left(\xi_{1}, \xi_{N_{2}}\right)\right) .
\end{aligned}
$$

Consequently,

$$
\sum_{j=0}^{n_{2}}(-1)^{j} \widehat{m}_{j}\left(\xi_{1}^{\prime}, \xi_{N_{2}}\right)>\sum_{j=0}^{n_{2}}(-1)^{j} \widehat{m}_{j}\left(\xi_{1}, \xi_{N_{2}}\right),
$$

and

$$
\frac{\partial}{\partial \xi_{1}} \sum_{j=0}^{n_{2}}(-1)^{j} \widehat{m}_{j}\left(\xi_{1}, \xi_{N_{2}}\right)>0 .
$$

Similarly, one obtains

$$
\frac{\partial}{\partial \xi_{N_{2}}} \sum_{j=0}^{n_{2}}(-1)^{j} \widehat{m}_{j}\left(\xi_{1}, \xi_{N_{2}}\right)>0
$$

and this completes the proof of the claim.

Since $\xi_{1}(s)$ increases at constant speed as $s$ decreases, there will be a point $s_{0}>0$ where two $\xi_{j}$ 's coincide: either $\xi_{1}\left(s_{0}\right)=\xi_{2}\left(s_{0}\right)$, or $\xi_{N_{2}}\left(s_{0}\right)=\xi_{N_{2}-1}\left(s_{0}\right)$. We define $s_{0}$ as the first point where one of these collisions occurs. Setting

$$
d \mu_{s}(x)=\frac{1}{c(s)}\left|\mathcal{R}^{1 / 2}(x) h(x ; s)\right| d x, \quad x \in\left[a_{1}, a_{2}\right] \cup\left[a_{3}, a_{4}\right],
$$


we have $\mu_{1}=\mu_{V}$ and $\mu_{s_{0}}$ corresponds to $h\left(. ; s_{0}\right)$ with at most $N_{1}+N_{2}+N_{3}-2$ real zeros of odd multiplicity. It remains to prove that $\mu_{s}$ is an equilibrium measure for all $s \in\left[s_{0}, 1\right]$. As before, we will use Lemma 7.1 to prove this.

We have

$$
\begin{aligned}
& m_{j}(s)=\int_{\eta_{j}}^{\eta_{j+1}}\left|\mathcal{R}^{1 / 2}(x) h(x ; s)\right| d x=\int_{\eta_{j}}^{\eta_{j+1}}\left|\mathcal{R}^{1 / 2}(x) h(x) f(x ; s)\right| d x \\
& \tilde{m}_{j}(s)=\int_{\gamma_{j+1}}^{\gamma_{j}}\left|\mathcal{R}^{1 / 2}(x) h(x) f(x ; s)\right| d x \\
& \widehat{m}_{j}(s)=\int_{\xi_{j}}^{\xi_{j+1}}\left|\mathcal{R}^{1 / 2}(x) h(x) f(x ; s)\right| d x
\end{aligned}
$$

where

$$
f(x ; s)=\frac{x-\xi_{1}(s)}{x-\xi_{1}} \frac{x-\xi_{N_{2}}(s)}{x-\xi_{N_{2}}}, \quad \xi_{1}<\xi_{1}(s)<\xi_{N_{2}}(s)<\xi_{N_{2}} .
$$

This function is bigger than 1 and increasing for $x<\xi_{1}$, and decreasing and bigger than 1 for $x>\xi_{2}$. This implies the inequalities

$$
\begin{aligned}
& m_{j}(s) \geq m_{j} f\left(\eta_{j+1} ; s\right), \quad m_{j}(s) \leq m_{j} f\left(\eta_{j} ; s\right), \quad 0 \leq j \leq N_{3}-1, \\
& \tilde{m}_{j}(s) \geq \tilde{m}_{j} f\left(\gamma_{j+1} ; s\right), \quad \tilde{m}_{j}(s) \leq \tilde{m}_{j} f\left(\gamma_{j} ; s\right), \quad 0 \leq j \leq N_{1}-1 .
\end{aligned}
$$

For $k$ even, we have

$$
\begin{aligned}
m_{0}(s) & -m_{1}(s)+m_{2}(s)-\ldots-m_{k-1}(s) \\
& \geq f\left(\eta_{1} ; s\right)\left(m_{0}-m_{1}\right)+f\left(\eta_{3} ; s\right)\left(m_{2}-m_{3}\right)+\ldots+f\left(\eta_{k-1} ; s\right)\left(m_{k-2}-m_{k-1}\right) \\
& \geq\left(m_{0}-m_{1}\right)+\left(m_{2}-m_{3}\right)+\ldots+\left(m_{k-2}-m_{k-1}\right) \\
& =\sum_{j=0}^{k-1}(-1)^{j} m_{j}>0,
\end{aligned}
$$

by Lemma 7.1 , and

$$
\begin{aligned}
\tilde{m}_{0}(s) & -\tilde{m}_{1}(s)+\tilde{m}_{2}(s)-\ldots-\tilde{m}_{k-1}(s) \\
& \geq f\left(\gamma_{1} ; s\right)\left(\tilde{m}_{0}-\tilde{m}_{1}\right)+f\left(\gamma_{3} ; s\right)\left(\tilde{m}_{2}-\tilde{m}_{3}\right)+\ldots+f\left(\gamma_{k-1} ; s\right)\left(\tilde{m}_{k-2}-\tilde{m}_{k-1}\right) \\
& \geq\left(\tilde{m}_{0}-\tilde{m}_{1}\right)+\left(\tilde{m}_{2}-\tilde{m}_{3}\right)+\ldots+\left(\tilde{m}_{k-2}-\tilde{m}_{k-1}\right) \\
& =\sum_{j=0}^{k-1}(-1)^{j} \tilde{m}_{j}>0 .
\end{aligned}
$$

For $\xi_{1}<x<\xi_{N_{2}}, f(x)<1$ and $f$ has a unique local maximum $\xi^{*}$. Suppose $\xi^{*}$ lies in between $\xi_{\ell}$ and $\xi_{\ell+2}$ with $\ell$ odd. Then, consider for $1 \leq d \leq \ell$ and $d$ odd the alternating sum

$$
\sum_{j=0}^{d}(-1)^{j} \widehat{m}_{j}(s)=\widehat{m}_{0}(s)-\left(\widehat{m}_{1}(s)-\widehat{m}_{2}(s)\right)-\ldots-\left(\widehat{m}_{d-2}(s)-\widehat{m}_{d-1}(s)\right)-\widehat{m}_{d}(s) .
$$

We have

$$
\begin{array}{r}
\widehat{m}_{2 j+1}(s)-\widehat{m}_{2 j+2}(s) \leq f\left(\xi_{2 j+2} ; s\right)\left(\widehat{m}_{2 j+1}-\widehat{m}_{2 j+2}\right) \\
<\widehat{m}_{2 j+1}-\widehat{m}_{2 j+2}, \quad j \geq 0, \xi_{2 j+2} \leq \xi^{*} .
\end{array}
$$


Furthermore, since $f$ is bigger than 1 outside $\left[\xi_{1}, \xi_{N_{2}}\right]$ and smaller inside, $\widehat{m}_{0}(s) \geq \widehat{m}_{0}$ and $\widehat{m}_{d}(s) \leq \widehat{m}_{d}$. Combining those inequalities, we obtain

$$
\sum_{j=0}^{d}(-1)^{j} \widehat{m}_{j}(s) \geq \sum_{j=0}^{d}(-1)^{j} \widehat{m}_{j}>0
$$

For $d$ odd and $\ell+2 \leq d \leq n_{2}-1$, we have by $(7.13)$,

$$
\sum_{j=0}^{d}(-1)^{j} \widehat{m}_{j}(s)=\sum_{j=d+1}^{n_{2}}(-1)^{j+1} \widehat{m}_{j}(s)
$$

But

$$
\begin{aligned}
\sum_{j=d+1}^{n_{2}}(-1)^{j+1} \widehat{m}_{j}(s) & =\widehat{m}_{n_{2}}(s)-\left(\widehat{m}_{n_{2}-1}(s)-\widehat{m}_{n_{2}-2}(s)\right)-\ldots-\widehat{m}_{d+1}(s) \\
& \geq \widehat{m}_{n_{2}}-\left(\widehat{m}_{n_{2}-1}-\widehat{m}_{n_{2}-2}\right)-\ldots-\widehat{m}_{d+1} \\
& =\sum_{j=0}^{d}(-1)^{j} \widehat{m}_{j}>0 .
\end{aligned}
$$

Case 2: $N_{2}=1$

We assume $N_{1}+N_{2}+N_{3}>1$ and $N_{2}=1$. Consequently, either $N_{1}$ or $N_{3}$ is strictly positive. We assume $N_{3}>0$, the other case is similar. Define a deformation of $h$ as follows:

$$
h(z)=\left(z-\xi_{1}(s)\right)\left(z-\eta_{1}(s)\right) \prod_{j=1}^{N_{1}}\left(z-\gamma_{j}\right) . \prod_{j=2}^{N_{3}}\left(z-\eta_{j}\right) \cdot \prod_{j=1}^{N_{4}}\left(z-z_{j}\right)\left(z-\bar{z}_{j}\right),
$$

where

$$
\eta_{1}(s)=s \eta_{1}+(1-s) \eta_{2}, \quad s \in[0,1],
$$

and where we choose $\xi_{1}(s)$ to be the unique value in $\left(a_{2}, a_{3}\right)$ such that $(7.13)$ holds. Similarly as in case 1 , one proves that $\xi_{1}(s) \geq \xi_{1}$ for $s \in[0,1]$. We will prove that

$$
d \mu_{s}(x)=\frac{1}{i c(s)} \mathcal{R}_{+}^{1 / 2}(x) h(x ; s) d x, \quad x \in\left[a_{1}, a_{2}\right] \cup\left[a_{3}, a_{4}\right],
$$

is an equilibrium measure for any $s \in[0,1]$, by Lemma 7.1 .

Note first that equation 7.13$)$ holds for any $s \in[0,1]$ by definition of $\xi_{1}(s)$, and that $(7.12)$ is trivially satisfied since $N_{2}=1$. We still need to prove (7.10)-(7.11) for $s \in[0,1]$. Note that

$$
\begin{aligned}
& m_{j}(s)=\int_{\eta_{j}}^{\eta_{j+1}}\left|\mathcal{R}^{1 / 2}(x) h(x ; s)\right| d x=\int_{\eta_{j}}^{\eta_{j+1}}\left|\mathcal{R}^{1 / 2}(x) h(x) f(x ; s)\right| d x \\
& \tilde{m}_{j}(s)=\int_{\gamma_{j+1}}^{\gamma_{j}}\left|\mathcal{R}^{1 / 2}(x) h(x) f(x ; s)\right| d x
\end{aligned}
$$


with

$$
f(x ; s)=\frac{x-\eta_{1}(s)}{x-\eta_{1}} \frac{x-\xi_{1}(s)}{x-\xi_{1}} .
$$

First, $f$ is increasing and bigger than 1 for $x<\xi_{1}$, which means that, for $k$ even,

$$
\begin{array}{r}
\sum_{j=0}^{k-1}\left(-1^{j}\right) \tilde{m}_{j}(s) \geq f\left(\gamma_{1}\right)\left(\tilde{m}_{0}-\tilde{m}_{1}\right)+f\left(\gamma_{3}\right)\left(\tilde{m}_{2}-\tilde{m}_{3}\right) \\
+\ldots+f\left(\gamma_{k-1}\right)\left(\tilde{m}_{k-2}-\tilde{m}_{k-1}\right) \\
\geq \sum_{j=0}^{k-1}\left(-1^{j}\right) \tilde{m}_{j}>0, \quad(7.46)
\end{array}
$$

where $\tilde{m}_{j}=\tilde{m}_{j}(1)$. Note that $\widehat{m}_{0}(s) \geq \widehat{m}_{0}$ for $s \in[0,1]$, which implies by 7.13 that $\widehat{m}_{1}(s) \geq \widehat{m}_{1}$. But $\widehat{m}_{1}(s) \leq f\left(a_{3} ; s\right) \widehat{m}_{1}$, which implies that $f\left(a_{3} ; s\right)>1$. We thus have that $f$ is increasing and bigger than 1 for $a_{3}<x<\eta_{1}$, which implies that $m_{0}(s)>m_{0}$ for $0<s<1$. Now, since for $x>\eta_{1}, f$ is increasing and smaller than 1 , we have

$$
\begin{array}{r}
\sum_{j=0}^{k-1}\left(-1^{j}\right) m_{j}(s) \geq m_{0}-f\left(\eta_{2}\right)\left(m_{1}-m_{2}\right)-f\left(\eta_{4}\right)\left(m_{3}-m_{4}\right)-\ldots-f\left(\eta_{k}\right) m_{k-1} \\
\geq \sum_{j=0}^{k-1}\left(-1^{j}\right) m_{j}>0 .
\end{array}
$$

This completes the proof of Theorem 2.9

\section{Time-derivatives of $\log Z_{N}$ in the two-cut regime}

In this section, we will extend the asymptotic results for the partition function $Z_{N}=$ $Z_{N}(V)$ to general two-cut regular polynomial external fields $V$. In order to accomplish this, we will first establish formula (8.6) for a logarithmic derivative (with respect to the parameters $\left\{t_{k}\right\}$ ) of the partition function, in terms of the solution $X$ to the $\mathrm{RH}$ problem for orthogonal polynomials on the full real line. The formula is valid for any potential, whether it is symmetric or not (or even regardless of the number of intervals in the support of the equilibrium measure). The asymptotic behavior of the solution $X$ as described in Section 8.2 is well understood. The real challenge is to obtain formulae that are simple and intrinsic, and this is aim of Sections 8.3 through 8.7

Let

$$
P_{j}(z)=\kappa_{j} z^{j}+\ldots, \quad j=0,1,2, \ldots,
$$

be the orthonormal polynomials with respect to the weight $e^{-N V_{\vec{t}}}$ on $\mathbb{R}$, where, as before,

$$
V(z)=V_{\vec{t}}(z)=V_{0}(z)+\sum_{j=1}^{2 d} t_{j} z^{j}, \quad V_{0}(z)=z^{4}-4 z^{2} .
$$

These polynomials satisfy a three term recurrence relation of the form

$$
z P_{k}(z)=a_{k} P_{k+1}(z)+b_{k} P_{k}(z)+a_{k-1} P_{k-1}(z) .
$$

Recall that $Z_{N}(V)$ is given by (1.1). 


\subsection{Differential identity}

For $z \in \mathbb{C} \backslash \mathbb{R}$, define the matrix-valued function

$$
X(z)=X^{(N)}(z ; \vec{t})=\left(\begin{array}{cc}
\kappa_{N}^{-1} P_{N}(z) & \frac{1}{2 \pi i} \kappa_{N}^{-1} \int_{\mathbb{R}} P_{N}(s) \frac{e^{-N V_{\vec{t}}(s)} d s}{s-z} \\
-2 \pi i \kappa_{N-1} P_{N-1}(z) & -\kappa_{N-1} \int_{\mathbb{R}} P_{N-1}(s) \frac{e^{-N V_{\vec{t}}^{(s)}} d s}{s-z}
\end{array}\right),
$$

which solves the standard RH problem for orthogonal polynomials [29]. In particular $X$ satisfies the jump relation

$$
X_{+}(x)=X_{-}(x)\left(\begin{array}{cc}
1 & e^{-N V_{\vec{t}}(x)} \\
0 & 1
\end{array}\right), \quad \text { for } x \in \mathbb{R} .
$$

Following [5, 44, we can derive an identity for $\frac{\partial}{\partial t_{k}} \log Z_{N}$. Therefore, recall the derivation of (3.17) in Section 3.2. In exactly the same way as we did there for the $\alpha$-derivative, we can prove that

$$
\begin{aligned}
\frac{d}{d t_{k}} \log Z_{N}(\vec{t})=-\int_{\mathbb{R}} \frac{\partial}{\partial t_{k}}\left(a_{N-1}(\vec{t}) P_{N}^{\prime}(x ; \vec{t}) P_{N-1}(x ; \vec{t})\right) e^{-N V_{\vec{t}}(x)} d x & \\
& +\int_{\mathbb{R}} \frac{\partial}{\partial t_{k}}\left(a_{N-1}(\vec{t}) P_{N}(x ; \vec{t}) P_{N-1}^{\prime}(x ; \vec{t})\right) e^{-N V_{\vec{t}}(x)} d x,
\end{aligned}
$$

where' denotes derivative with respect to $x$. Integrating by parts, we obtain

$$
\begin{aligned}
\frac{d}{d t_{k}} \log Z_{N}(\vec{t})=-N \int_{\mathbb{R}} a_{N-1}(\vec{t})\left(P_{N}^{\prime}(x ; \vec{t})\right. & P_{N-1}(x ; \vec{t}) \\
& \left.-P_{N}(x ; \vec{t}) P_{N-1}^{\prime}(x ; \vec{t})\right) x^{k} e^{-N V_{\vec{t}}(x)} d x .
\end{aligned}
$$

By $(8.2)$ and the jump relation $(8.3)$, it is straightforward to see that

$$
\begin{aligned}
\frac{d}{d t_{k}} \log Z_{N}(\vec{t}) & =-\frac{N}{2 \pi i} \int_{\mathbb{R}}\left(X_{ \pm}^{-1} X_{ \pm}^{\prime}\right)_{21}(x) x^{k} e^{-N V_{\vec{t}}(x)} d x \\
& =\frac{N}{4 \pi i} \int_{\mathbb{R}}\left[\operatorname{Tr}\left(X_{+}^{-1} X_{+}^{\prime} \sigma_{3}\right)(x)-\operatorname{Tr}\left(X_{-}^{-1} X_{-}^{\prime} \sigma_{3}\right)(x)\right] x^{k} d x
\end{aligned}
$$

where' denotes derivative with respect to $x$. Using a contour deformation argument, we can write the integral over the real line as the limit of the integral of $\operatorname{Tr}\left(X^{-1}(z) X^{\prime}(z) \sigma_{3}\right)$ over a large clockwise oriented circle in the complex plane, as the radius tends to infinity. Although $z^{k} \operatorname{Tr}\left(X^{-1}(z) X^{\prime}(z) \sigma_{3}\right)$ is not analytic at $\infty$, it has a large $z$ expansion given by

$$
z^{k} \operatorname{Tr}\left(X^{-1}(z) X^{\prime}(z) \sigma_{3}\right)=\sum_{j=-1}^{k-2} c_{j} z^{j}+\mathcal{O}\left(z^{-2}\right), \quad z \rightarrow \infty
$$

for any $k \in \mathbb{N}$, where $-c_{-1}$ is the residue at infinity of $z^{k} \operatorname{Tr}\left(X^{-1}(z) X^{\prime}(z) \sigma_{3}\right)$. By residue calculus, we obtain 33 ]

$$
\frac{d}{d t_{k}} \log Z_{N}(\vec{t})=\frac{N}{2} \operatorname{Res}_{z=\infty}\left[\operatorname{Tr}\left(X^{-1}(z) X^{\prime}(z) \sigma_{3}\right) z^{k}\right] .
$$

This identity is not new; similar identities can be found for example in [5, 33, 44]. 


\subsection{Large $N$ asymptotics for $X$}

We will now derive large $N$ asymptotics for the right hand side of (8.8), and integrate it afterwards along a two-cut regular path in the $\vec{t}$-space as constructed in Section 7 . Large $N$ asymptotics for the matrix $X(z)$ have been obtained in [21, 22] for general $k$ cut regular polynomials $V=V_{\vec{t}}$. In this section, we recall the results from [21, 22] in the two-cut regular case, where the equilibrium measure $\mu_{V}$ is supported on $\left[a_{1}, a_{2}\right] \cup\left[a_{3}, a_{4}\right]$ with $a_{1}<a_{2}<a_{3}<a_{4}$. We write

$$
\Omega=\int_{a_{3}}^{a_{4}} d \mu_{V}(x)
$$

i.e. $\Omega$ is the expected portion of random matrix eigenvalues in the rightmost interval of the support as $N \rightarrow \infty$, and

$$
g(z)=\int \log (z-s) d \mu_{V}(s) .
$$

The equilibrium measure $\mu_{V}$ can be written in the form [20]

$$
d \mu_{V}(x)=\psi(x) d x=\frac{\mathcal{R}(x)_{+}^{1 / 2}}{\pi i} h(x) d x, \quad \text { for } x \in\left[a_{1}, a_{2}\right] \cup\left[a_{3}, a_{4}\right],
$$

with $h$ given by

$$
h(z)=-\frac{1}{2 \pi i} f_{J} \frac{V^{\prime}(\eta)}{\mathcal{R}(\eta)_{+}^{1 / 2}(\eta-z)} d \eta, \quad J=\left[a_{1}, a_{2}\right] \cup\left[a_{3}, a_{4}\right],
$$

where $V(z)=V_{\vec{t}}(z)$ is the polynomial external field and

$$
\mathcal{R}(z)=\prod_{j=1}^{4}\left(z-a_{j}\right)
$$

The square root $\mathcal{R}(z)^{1 / 2}$ is as usual chosen in such a way that it is positive for $z>a_{4}$ and analytic in $\mathbb{C} \backslash\left[a_{1}, a_{2}\right] \cup\left[a_{3}, a_{4}\right] . \mathcal{R}_{+}(z)^{1 / 2}$ denotes the boundary value from the upper half plane on $\left[a_{1}, a_{2}\right] \cup\left[a_{3}, a_{4}\right]$.

Remark 8.1 There is a difference in notation between the function $h$ here and the one in Section 7. they differ by a constant factor. The function $\frac{1}{c} h$ from Section 7, where it was convenient to consider a monic polynomial $h$, is equal to $\frac{1}{\pi} h$ using the notation of this section, where we prefer to have $h$ defined by 8.12 .

The function $h(z)$ is a polynomial, which also has the property of being the polynomial part of the function $\frac{V^{\prime}(z)}{\mathcal{R}(z)^{1 / 2}}$ at infinity, i.e.

$$
\frac{V^{\prime}(z)}{\mathcal{R}(z)^{1 / 2}}=h(z)+\mathcal{O}\left(z^{-1}\right), \quad z \rightarrow \infty .
$$

The endpoints $a_{1}<a_{2}<a_{3}<a_{4}$ are determined by

$$
\int_{\gamma} \frac{V^{\prime}(\lambda) \lambda^{k}}{\mathcal{R}(\lambda)^{1 / 2}} d \lambda=0, \quad k=0,1, \quad-\frac{1}{4 \pi i} \int_{\gamma} \frac{V^{\prime}(\lambda) \lambda^{2}}{\mathcal{R}(\lambda)^{1 / 2}} d \lambda=1,
$$


where $\gamma$ is a clock-wise oriented contour surrounding the support $J$, and by the normalization condition

$$
\int_{a_{2}}^{a_{3}} \mathcal{R}(\lambda)^{1 / 2} h(\lambda) d \lambda=0 .
$$

These 4 equations determine $a_{1}<a_{2}<a_{3}<a_{4}$ uniquely as a function of $t_{1}, \ldots, t_{2 d}$.

For $z$ outside small disks surrounding the endpoints $a_{1}, \ldots, a_{4}$, the matrix $X(z)$ defined in 8.2 can be expressed as [21]

$$
X(z)=R(z) P^{\infty}(z) e^{N g(z) \sigma_{3}} .
$$

Here:

(1) $R(z)$ is a $2 \times 2$ matrix that solves the so-called error $\mathrm{RH}$ problem and admits an asymptotic expansion of the form

$$
R(z)=I+\sum_{j=1}^{k} N^{-j} R^{(j)}(z)+\mathcal{O}\left(N^{-k-1}\right), \quad N \rightarrow \infty,
$$

where $R^{(j)}(z), j=1,2, \ldots$ can be computed by a recursive procedure and remains bounded as $N \rightarrow \infty$, and

(2) $P^{\infty}(z)$ is the unique solution to the following $\mathrm{RH}$ problem:

\section{RH problem for $P^{\infty}(z)$}

(a) $P^{\infty}(z)$ is a $2 \times 2$ matrix analytic in $\mathbb{C} \backslash\left[a_{1}, a_{4}\right]$.

(b) $P^{\infty}$ has the jumps

$$
\begin{gathered}
P_{+}^{\infty}(z)=P_{-}^{\infty}(z)\left(\begin{array}{cc}
0 & 1 \\
-1 & 0
\end{array}\right), \quad z \in\left(a_{1}, a_{2}\right) \cup\left(a_{3}, a_{4}\right), \\
P_{+}^{\infty}(z)=P_{-}^{\infty}(z) e^{-2 \pi i N \Omega \sigma_{3}}, \quad z \in\left(a_{2}, a_{3}\right) . \\
\text { (c) } P^{\infty}(z)=I+\mathcal{O}\left(z^{-1}\right) \text { as } z \rightarrow \infty . \\
\text { (d) As } z \rightarrow a_{j}, j=1, \ldots, 4 \text {, we have } P^{\infty}(z)=\mathcal{O}\left(\left|z-a_{j}\right|^{-1 / 4}\right) .
\end{gathered}
$$

This RH problem can be solved using elliptic $\theta$-functions (see e.g. [36],[19],[22]), we will give more details about this construction later on.

Using 8.8, 8.17, and the identity [41]

$$
\frac{\partial}{\partial t_{k}} F_{0}=-\operatorname{Res}_{z=\infty}\left(z^{k} g^{\prime}(z)\right)
$$

where

$$
F_{0}=\iint \log |x-y|^{-1} d \mu_{V}(x) d \mu_{V}(y)+\int V(x) d \mu_{V}(x),
$$

we obtain

$$
\begin{aligned}
\frac{\partial}{\partial t_{k}} \log Z_{N}=-N^{2} \frac{\partial}{\partial t_{k}} F_{0} & +\frac{N}{2} \operatorname{Res}_{z=\infty} \operatorname{Tr}\left(P^{\infty}(z)^{-1} P^{\infty}(z)^{\prime} \sigma_{3} z^{k}\right) \\
& +\frac{N}{2} \operatorname{Res}_{z=\infty} \operatorname{Tr}\left(P^{\infty}(z)^{-1} R^{-1}(z) R^{\prime}(z) P^{\infty}(z) \sigma_{3} z^{k}\right) .
\end{aligned}
$$


Substituting (8.18), we obtain large $N$ asymptotics for the right hand side of this identity:

$$
\begin{aligned}
\frac{\partial}{\partial t_{k}} \log Z_{N}=-N^{2} \frac{\partial}{\partial t_{k}} F_{0}+\frac{N}{2} \operatorname{Res}_{z=\infty} \operatorname{Tr}\left(P^{\infty}(z)^{-1} P^{\infty}(z)^{\prime} \sigma_{3} z^{k}\right) \\
\quad+\frac{1}{2} \operatorname{Res}_{z=\infty} \operatorname{Tr}\left(P^{\infty}(z)^{-1} \frac{d R^{(1)}(z)}{d z} P^{\infty}(z) \sigma_{3} z^{k}\right)+\mathcal{O}\left(N^{-1}\right), \quad N \rightarrow \infty
\end{aligned}
$$

Here we used the fact that $P^{\infty}(z)$ is uniformly bounded for $N$ and $z$ large. This will follow from our construction of $P^{\infty}$ below. We observe that the second term at the right hand side of the above formula is invariant under the transformation

$$
P^{\infty}(z) \mapsto C P^{\infty}(z)
$$

where $C$ is an invertible matrix. If in addition we apply the transformation

$$
R^{(1)}(z) \mapsto C R^{(1)}(z) C^{-1},
$$

the third term on the right hand side of (8.27) is also unchanged. Let $C=P^{\infty}\left(z_{0}\right)^{-1}$, and define

$$
P^{\infty}\left(z, z_{0}\right) \equiv P^{\infty}\left(z_{0}\right)^{-1} P^{\infty}(z), \quad z_{0} \neq a_{j}, \quad j=1,2,3,4 .
$$

Now, $P^{\infty}\left(z, z_{0}\right)$ solves the RH conditions 8.19 , (8.20), 8.21) and (8.23), with the asymptotic condition 8.22 replaced by

$$
P^{\infty}\left(z, z_{0}\right)=I+\mathcal{O}\left(z-z_{0}\right), \quad \text { as } z \rightarrow z_{0} .
$$

$P^{\infty}\left(z, z_{0}\right)$ is moreover uniquely determined by those conditions. It will be convenient for us to construct the solution to this more general RH problem for $P^{\infty}\left(z, z_{0}\right)$, normalized at $z_{0}$, instead of the particular case of $P^{\infty}(z)=P^{\infty}(z, \infty)$. Writing

$$
\begin{gathered}
R\left(z, z_{0}\right)=P^{\infty}\left(z_{0}\right)^{-1} R(z) P^{\infty}\left(z_{0}\right), \quad R^{(1)}\left(z, z_{0}\right) \equiv P^{\infty}\left(z_{0}\right)^{-1} R^{(1)}(z) P^{\infty}\left(z_{0}\right), \\
\operatorname{Res}_{z=\infty} \operatorname{Tr}\left(P^{\infty}(z)^{-1} \frac{d R^{(1)}(z)}{d z} P^{\infty}(z) \sigma_{3} z^{k}\right) \\
=\operatorname{Res}_{z=\infty} \operatorname{Tr}\left(P^{\infty}\left(z, z_{0}\right)^{-1} \frac{d R^{(1)}\left(z, z_{0}\right)}{d z} P^{\infty}\left(z, z_{0}\right) \sigma_{3} z^{k}\right) .
\end{gathered}
$$

Since the left hand side in this equation is independent of $z_{0}$, we can let $z_{0} \rightarrow z$ at the right hand side, and obtain

$$
\begin{aligned}
\operatorname{Res}_{z=\infty} \operatorname{Tr}\left(P^{\infty}(z)^{-1} \frac{d R^{(1)}(z)}{d z} P^{\infty}(z) \sigma_{3} z^{k}\right) & \\
& =\operatorname{Res}_{z=\infty} \operatorname{Tr}\left(\left.\frac{d R^{(1)}\left(z, z_{0}\right)}{d z}\right|_{z_{0}=z} \sigma_{3} z^{k}\right) .
\end{aligned}
$$

Therefore, the relation (8.27) can be rewritten in the form 
Lemma 8.2 We have

$$
\begin{aligned}
\frac{\partial}{\partial t_{k}} \log Z_{N}\left(V_{\vec{t}}\right) & =-N^{2} \frac{\partial}{\partial t_{k}} F_{0}+\frac{N}{2} \operatorname{Res}_{z=\infty} \operatorname{Tr}\left(\left.\frac{d P^{\infty}\left(z, z_{0}\right)}{d z}\right|_{z_{0}=z} \sigma_{3} z^{k}\right) \\
+ & \frac{1}{2} \operatorname{Res}_{z=\infty} \operatorname{Tr}\left(\left.\frac{d R^{(1)}\left(z, z_{0}\right)}{d z}\right|_{z_{0}=z} \sigma_{3} z^{k}\right)+\mathcal{O}\left(N^{-1}\right), \quad N \rightarrow \infty,
\end{aligned}
$$

where $P^{\infty}\left(z, z_{0}\right)$ satisfies the $R H$ conditions (8.19)-(8.21), (8.23), and the normalization 8.29), and $R^{(1)}\left(z, z_{0}\right)$ is defined in 8.30).

The above Lemma demonstrates the asymptotic behavior of the partition function for general two-cut potentials. Moreover, it is clear from the Riemann-Hilbert analysis described that the asymptotic behavior can be determined to any desired order. While a representation in terms of quantities derived from Riemann-Hilbert analysis is to some the end of the road, the goal of a representation in terms of other intrinsic quantities is fundamental. In Subsections 8.3 and 8.4 , we will construct $P^{\infty}\left(z, z_{0}\right)$ and $R^{(1)}\left(z, z_{0}\right)$ explicitly, and in the remaining Subsections we will throw the full force of the theory of Riemann surfaces at these formulae so as to arrive at a canonical expansion, which has not appeared in the literature before.

\subsection{The elliptic Riemann surface and the construction of $P^{\infty}\left(z, z_{0}\right)$}

Instead of constructing $P^{\infty}(z)$ with normalization at $\infty$, we will directly construct the unique matrix function $P^{\infty}\left(z, z_{0}\right)$ which satisfies the $\mathrm{RH}$ conditions 8.19)-(8.21), (8.23), and the normalization

$$
P^{\infty}\left(z, z_{0}\right)=I+\mathcal{O}\left(z-z_{0}\right), \quad \text { as } z \rightarrow z_{0} .
$$

If $z_{0}=\infty$, the solution $P^{\infty}\left(z, z_{0}\right)$ is equal to $P^{\infty}(z)$. In order to construct $P^{\infty}\left(z, z_{0}\right)$, we will make use of the elliptic Riemann surface

$$
\mathcal{S} \equiv\left\{(z, y) \in \mathbb{C}^{2} \cup\{(\infty, \pm \infty)\}: f(z, y)=y^{2}-\prod_{j=1}^{4}\left(z-a_{j}\right)=0\right\}
$$

This surface consists of two sheets glued together along the cuts $\left[a_{1}, a_{2}\right]$ and $\left[a_{3}, a_{4}\right]$. Given a point $Q=(z, y)$ on $\mathcal{S}$, we write $z=z(Q)$ for its projection on the complex plane. For each $z \in \mathbb{C} \cup\{\infty\} \backslash\left\{a_{1}, a_{2}, a_{3}, a_{4}\right\}$, the pre-image of the projection map gives two points $(z, y)$ and $(z,-y)$, one on each sheet of the Riemann surface, which we will denote below by $z^{(1)}$ and $z^{(2)}$ and which we call conjugate points on the surface. We adopt the convention that $\infty^{(1)}=(\infty,+\infty), \infty^{(2)}=(\infty,-\infty)$.

Let us introduce a basis of canonical cycles $\{\alpha, \beta\}$ as in Figure 1. The holomorphic 1 -form on $\mathcal{S}$ is given by $\frac{d z}{y}$, and we define the $\alpha$ - and $\beta$-periods $\mathcal{A}$ and $\mathcal{B}$ of this 1 -form by

$$
\mathcal{A}=\oint_{\alpha} \frac{d z}{y}, \quad \mathcal{B}=\oint_{\beta} \frac{d z}{y} .
$$

Then the normalized holomorphic 1-form is given by

$$
v=\frac{d z}{\mathcal{A} y}
$$


and satisfies the condition $\oint_{\alpha} v=1$. Writing $B=\frac{\mathcal{B}}{\mathcal{A}}$, we have $\operatorname{Im} B>0$. The $\theta$-function with characteristics $\delta, \epsilon \in \mathbb{R}$ is given by (2.27) and satisfies the periodicity properties

$$
\begin{aligned}
& \theta\left[\begin{array}{l}
\delta \\
\epsilon
\end{array}\right](z+1 ; B)=\theta\left[\begin{array}{l}
\delta \\
\epsilon
\end{array}\right](z ; B) e^{2 \pi i \delta}, \\
& \theta\left[\begin{array}{l}
\delta \\
\epsilon
\end{array}\right](z+B ; B)=\theta\left[\begin{array}{l}
\delta \\
\epsilon
\end{array}\right](z ; B) e^{-i \pi(B+2 z+2 \epsilon)},
\end{aligned}
$$

and the heat equation

$$
\frac{\partial^{2} \theta\left[\begin{array}{l}
\delta \\
\epsilon
\end{array}\right](z ; B)}{\partial z^{2}}=4 \pi i \frac{\partial \theta\left[\begin{array}{l}
\delta \\
\epsilon
\end{array}\right](z ; B)}{\partial B} .
$$

The RH solution $P^{\infty}\left(z, z_{0}\right)$ will be built out of the following object which is related to the Szegö kernel [28]:

$$
\widehat{S}\left[\begin{array}{c}
\delta \\
\epsilon
\end{array}\right]\left(Q, Q_{0}\right)=\frac{1}{2}\left(\frac{\gamma(Q)}{\gamma\left(Q_{0}\right)}+\frac{\gamma\left(Q_{0}\right)}{\gamma(Q)}\right) \frac{\theta\left[\begin{array}{l}
\delta \\
\epsilon
\end{array}\right]\left(\int_{Q_{0}}^{Q} v ; B\right)}{\theta\left(\int_{Q_{0}}^{Q} v ; B\right)} \frac{\theta(0 ; B)}{\theta\left[\begin{array}{l}
\delta \\
\epsilon
\end{array}\right](0 ; B)},
$$

where $Q_{0}, Q \in \mathcal{S}$, and where $\gamma(Q), Q \in \mathcal{S}$, is defined as

$$
\gamma(Q)=\left(\frac{\left(z(Q)-a_{2}\right)\left(z(Q)-a_{4}\right)}{\left(z(Q)-a_{1}\right)\left(z(Q)-a_{3}\right)}\right)^{1 / 4}
$$

where $z(Q)$ is the projection of $Q$ to the complex plane. The function $\gamma$ is a multivalued function on the surface $\mathcal{S}$ and we define it in such a way that

$$
\gamma\left(z^{(2)}\right)=-i \gamma\left(z^{(1)}\right), \quad \gamma\left(\infty^{(1)}\right)=1 .
$$

On the cuts $\left(a_{1}, a_{2}\right)$ and $\left(a_{3}, a_{4}\right)$ oriented from left to right, we have

$$
\gamma\left(z^{(2)}\right)_{+}=\gamma\left(z^{(1)}\right)_{-}, \quad \gamma\left(z^{(2)}\right)_{-}=-\gamma\left(z^{(1)}\right)_{+} .
$$

Remark 8.3 The Szegö kernel with characteristics $\left[\begin{array}{l}\delta \\ \epsilon\end{array}\right]$ is defined as

$$
S\left[\begin{array}{l}
\delta \\
\epsilon
\end{array}\right]\left(Q, Q_{0}\right)=\widehat{S}\left[\begin{array}{l}
\delta \\
\epsilon
\end{array}\right]\left(Q, Q_{0}\right) \frac{\sqrt{d z(Q) d z\left(Q_{0}\right)}}{z(Q)-Z\left(Q_{0}\right)}
$$

and it is a $\left(\frac{1}{2}, \frac{1}{2}\right)$ form on $\mathcal{S} \times \mathcal{S}$ having only a simple pole on the diagonal as $Q \rightarrow Q_{0}$ [28]. It follows that $\widehat{S}\left[\begin{array}{l}\delta \\ \epsilon\end{array}\right]\left(Q, Q_{0}\right)$ has only singularities of branching type at the points $\left(a_{j}, 0\right), j=1,2,3,4$.

We have the following properties:

(i) As $Q_{0} \rightarrow Q$,

$$
\begin{aligned}
\widehat{S}\left[\begin{array}{l}
\delta \\
\epsilon
\end{array}\right]\left(Q, Q_{0}\right)=1+\left(\log \theta\left[\begin{array}{l}
\delta \\
\epsilon
\end{array}\right](0 ; B)\right)^{\prime} \frac{v(Q)}{d z(Q)} & \left(z(Q)-z\left(Q_{0}\right)\right) \\
& +\mathcal{O}\left(\left(z(Q)-z\left(Q_{0}\right)\right)^{2}\right),
\end{aligned}
$$


where $v$ is the normalized holomorphic 1-form. In the relation above and in the rest of the paper, we denote

$$
\left(\log \theta\left[\begin{array}{l}
\delta \\
\epsilon
\end{array}\right](0 ; B)\right)^{\prime}=\left.\frac{\partial}{\partial z} \log \theta\left[\begin{array}{l}
\delta \\
\epsilon
\end{array}\right](z ; B)\right|_{z=0},
$$

and similarly for higher order derivatives with respect to $z$.

(ii) As $Q$ goes around $\alpha$ and $\beta$-cycles one has

$$
\begin{aligned}
& T_{\alpha} \widehat{S}\left[\begin{array}{l}
\delta \\
\epsilon
\end{array}\right]\left(Q, Q_{0}\right)=\epsilon^{2 \pi i \delta} \widehat{S}\left[\begin{array}{l}
\delta \\
\epsilon
\end{array}\right]\left(Q, Q_{0}\right), \\
& T_{\beta} \widehat{S}\left[\begin{array}{l}
\delta \\
\epsilon
\end{array}\right]\left(Q, Q_{0}\right)=\epsilon^{-2 \pi i \epsilon} \widehat{S}\left[\begin{array}{l}
\delta \\
\epsilon
\end{array}\right]\left(Q, Q_{0}\right),
\end{aligned}
$$

where $T_{\alpha}$ and $T_{\beta}$ are the monodromy operators.

The solution $P^{\infty}\left(z, z_{0}\right)$ of the matrix RH problem 8.19), 8.20, 8.21), 8.23), normalized to the identity at $z_{0}$, can be expressed in terms of $\widehat{S}\left[\begin{array}{l}\delta \\ \epsilon\end{array}\right]\left(Q, Q_{0}\right)$. For $j, k=$ 1,2 and $z \in \mathbb{C} \backslash\left[a_{1}, a_{4}\right], z_{0} \in \mathbb{C} \cup\{\infty\}$, we have [36, 19, 38]

$$
P_{j k}^{\infty}\left(z, z_{0}\right)=\widehat{S}\left[\begin{array}{l}
\delta \\
\epsilon
\end{array}\right]\left(z^{(k)}, z_{0}^{(j)}\right), \quad \text { with } \epsilon=-N \Omega, \quad \delta=0,
$$

where $z^{(1)} \equiv(z, y)$ and $z_{0}^{(1)} \equiv\left(z_{0}, y_{0}\right)$ lie on the first sheet of the surface, and $z^{(2)} \equiv$ $(z,-y)$ and $z_{0}^{(2)} \equiv\left(z_{0},-y_{0}\right)$ on the second sheet. The integrals of the form

$$
\int_{z_{0}^{(1)}}^{z^{(2)}} v \text { or } \int_{z_{0}^{(2)}}^{z^{(1)}} v
$$

in 8.40 should be understood in the following way:

$$
\int_{z_{0}^{(1)}}^{z^{(2)}} v=\int_{z_{0}^{(1)}}^{a_{4}} v-\int_{a_{4}}^{z^{(1)}} v, \quad \int_{z_{0}^{(2)}}^{z^{(1)}} v=-\int_{z_{0}^{(1)}}^{a_{4}} v+\int_{a_{4}}^{z^{(1)}} v
$$

i.e. the point $a_{4}$ connects the parts of the integration path on the first and second sheet of the surface. The explicit formula for $P^{\infty}\left(z, z_{0}\right)$ thus takes the form

$$
\begin{aligned}
& P^{\infty}\left(z, z_{0}\right)=\frac{\theta(0 ; B)}{2 \theta(N \Omega ; B)} \times \\
& \left(\begin{array}{cc}
\left(\frac{\gamma(z)}{\gamma\left(z_{0}\right)}+\frac{\gamma\left(z_{0}\right)}{\gamma(z)}\right) \frac{\theta\left(\int_{z_{0}}^{z} v-N \Omega ; B\right)}{\theta\left(\int_{z_{0}}^{z} v ; B\right)} & \frac{\frac{\gamma(z)}{\gamma\left(z_{0}\right)}-\frac{\gamma\left(z_{0}\right)}{\gamma(z)} \theta\left(\int_{z_{0}}^{a_{4}} v-\int_{a_{4}}^{z} v-N \Omega ; B\right)}{i} \\
\frac{\frac{\gamma\left(z_{0}\right)}{\gamma(z)}-\frac{\gamma(z)}{\gamma\left(z_{0}\right)} \theta\left(-\int_{z_{0}}^{a_{4}} v+\int_{a_{4}}^{z} v-N \Omega ; B\right)}{i\left(\int_{z_{0}}^{a_{4}} v-\int_{z_{0}} v ; B\right)} & \left(\frac{\gamma(z)}{\gamma\left(z_{0}\right)}+\frac{\gamma\left(z_{0}\right)}{\gamma(z)}\right) \frac{\theta\left(\int_{z_{0}}^{z} v+N \Omega ; B\right)}{\theta\left(\int_{z_{0}}^{a_{4}} v ; B\right)}
\end{array}\right),
\end{aligned}
$$


where we now simply write $z$ and $z_{0}$ as complex variables instead of points on the Riemann surface. The complex function $\gamma$ is now defined as

$$
\gamma(z)=\left(\frac{\left(z-a_{2}\right)\left(z-a_{4}\right)}{\left(z-a_{1}\right)\left(z-a_{3}\right)}\right)^{1 / 4}
$$

with branch cuts on $\left[a_{1}, a_{2}\right] \cup\left[a_{3}, a_{4}\right]$ and such that $\gamma(\infty)=1$. When $z_{0}=\infty$, this formula for $P^{\infty}$ reduces to the one derived in [21]. From Fay's trisecant formula [28], or alternatively from the $\mathrm{RH}$ conditions, it follows that

$$
\operatorname{det} P^{\infty}\left(z, z_{0}\right) \equiv 1
$$

Furthermore one verifies easily that

$$
P^{\infty}\left(z, z_{0}\right)^{-1}=P^{\infty}\left(z_{0}, z\right)
$$

and that, changing the base point, one has the relation

$$
P^{\infty}\left(z, z_{1}\right)=P^{\infty}\left(z_{1}, z_{0}\right)^{-1} P^{\infty}\left(z, z_{0}\right)=P^{\infty}\left(z_{0}, z_{1}\right) P^{\infty}\left(z, z_{0}\right) .
$$

\subsection{Construction of $R^{(1)}\left(z, z_{0}\right)$}

It was shown in [21] that the matrix function $R=R(z)$ appearing in 8.17) is the solution to a small-norm RH problem similar to the one in Section 4.7, with jumps on a contour $\Sigma_{R}$ as shown in Figure 5 .

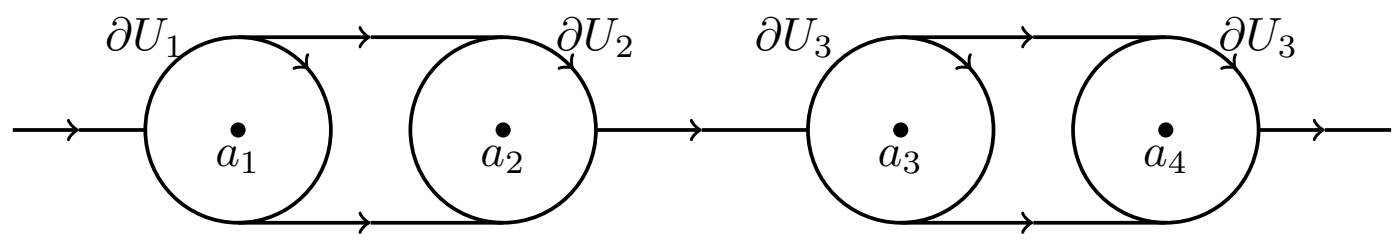

Figure 5: The jump contour $\Sigma_{R}$ for the error matrix $R$.

The only jumps for $R$ that are not exponentially close to the identity matrix, are the ones on clockwise oriented circles $\partial U_{1}, \partial U_{2}, \partial U_{3}, \partial U_{4}$ surrounding the endpoints $a_{1}, a_{2}, a_{3}, a_{4}$.

\section{RH problem for $R$}

(a) $R(z)$ is analytic for $z \in \mathbb{C} \backslash \Sigma_{R}$.

\footnotetext{
${ }^{1}$ The equivalence of the above formula with the one appearing in 21] is obtained by observing that the quantity $u_{+}(\infty)+d$ appearing in [21] on page 1367, formula (4.45), is identically zero.
} 
(b) For $z \in \Sigma_{R} \backslash\left(\cup_{m=1}^{4} \partial U_{m}\right)$, we have $R_{+}(z)=R_{-}(z)\left(I+\mathcal{O}\left(e^{-c N}\right)\right)$ as $N \rightarrow \infty$ with $c>0$; for $z \in \cup_{m=1}^{4} \partial U_{m}$, we have

$$
R_{+}(z)=R_{-}(z) J_{R}(z),
$$

where $J_{R}$ has an asymptotic expansion of the form

$$
J_{R}(z)=I+\sum_{j=1}^{k} \frac{\Delta^{(j)}(z)}{N^{j}}+\mathcal{O}\left(N^{-k-1}\right), \quad N \rightarrow \infty,
$$

for any $k \in \mathbb{N}$, and where $\Delta^{(j)}(z)$ depends on $N$ but is uniformly bounded as $N \rightarrow \infty$.

(c) As $z \rightarrow \infty$, we have

$$
R(z)=I+\mathcal{O}\left(z^{-1}\right) .
$$

Explicit formulas for $\Delta^{(j)}$ can be found. For that purpose, we need to define auxiliary objects that are related to the Airy RH problem as in Section 4.5. Let

$$
A_{j}=\frac{1}{2}\left(\frac{2}{3}\right)^{-j}\left(\begin{array}{cc}
(-1)^{j}\left(s_{j}+r_{j}\right) & i\left(s_{j}-r_{j}\right) \\
-i(-1)^{j}\left(s_{j}-r_{j}\right) & s_{j}+r_{j}
\end{array}\right), \quad j=1,2, \ldots,
$$

where $s_{j}$ and $r_{j}$ are defined in 4.43). For $j=1$, we have

$$
A_{1}=\frac{1}{8}\left(\begin{array}{cc}
\frac{1}{6} & i \\
i & -\frac{1}{6}
\end{array}\right) .
$$

The correction terms $\Delta^{(j)}, j=1,2, \ldots$ for the jump matrix $J_{R}$ can be expressed in terms of $A_{j}$ and are given by

$$
\Delta^{(j)}(z)= \begin{cases}\left(-f_{1}(z)\right)^{-\frac{3}{2} j} P^{(\infty)}(z) \sigma_{3} A_{j} \sigma_{3} P^{(\infty)}(z)^{-1}, & z \in \partial U_{1} \\ f_{2}(z)^{-\frac{3}{2} j} P^{(\infty)}(z) \mathcal{Q}(z) A_{j} \mathcal{Q}(z)^{-1} P^{(\infty)}(z)^{-1}, & z \in \partial U_{2} \\ \left(-f_{3}(z)\right)^{-\frac{3}{2} j} P^{(\infty)}(z) \mathcal{Q}(z) \sigma_{3} A_{j} \sigma_{3} \mathcal{Q}(z)^{-1} P^{(\infty)}(z)^{-1}, & z \in \partial U_{3} \\ f_{4}(z)^{-\frac{3}{2} j} P^{(\infty)}(z) A_{j} P^{(\infty)}(z)^{-1}, & z \in \partial U_{4}\end{cases}
$$

where

$$
\mathcal{Q}(z)=e^{ \pm \pi i N \Omega \sigma_{3}}, \quad \text { for } \pm \operatorname{Im} z>0,
$$

and where $f_{m}, m=1,2,3,4$, is a conformal map from a neighborhood $U_{m}$ of $a_{m}$ to a neighborhood of 0 , with $f_{m}^{\prime}\left(a_{m}\right)>0$. As $z \rightarrow a_{m}$, we have

$$
\left((-1)^{m} f_{m}(z)\right)^{3 / 2}=\widehat{\psi}\left(a_{m}\right)\left(z-a_{m}\right)^{3 / 2}+\frac{3}{2} \sum_{k=1}^{M} \widehat{\psi}^{(k)}\left(a_{m}\right) \frac{\left(z-a_{m}\right)^{k+\frac{3}{2}}}{k !\left(k+\frac{3}{2}\right)}+\mathcal{O}\left(\left(z-a_{m}\right)^{M+\frac{5}{2}}\right),
$$

where $\widehat{\psi}\left(a_{m}\right), \widehat{\psi}^{\prime}\left(a_{m}\right)$ and $\widehat{\psi}^{(k)}\left(a_{m}\right)$ are related to the equilibrium density $\psi$ and the function $h$, defined in (8.11), in the following way:

$$
\begin{aligned}
& \widehat{\psi}\left(a_{m}\right)=\lim _{z \rightarrow a_{m}^{+}} h(z) \prod_{j \neq m}\left(z-a_{j}\right)^{1 / 2}, \\
& \widehat{\psi}^{(k)}\left(a_{m}\right)=\lim _{z \rightarrow a_{m}^{+}} \frac{\partial^{k}}{\partial z^{k}}\left(h(z) \prod_{j \neq m}\left(z-a_{j}\right)^{1 / 2}\right), \quad m=1,2,3,4 .
\end{aligned}
$$


Here $z \rightarrow a_{m}^{+}$denotes the limit as $z \rightarrow a_{m}$ from the upper half plane, and $\left(z-a_{j}\right)^{1 / 2}$ is the principal branch of the square root with branch cut on $\left(-\infty, a_{j}\right]$ and positive for $z>a_{j}$. Note that

$$
\widehat{\psi}\left(a_{2}\right), \widehat{\psi}\left(a_{4}\right)>0, \quad \widehat{\psi}\left(a_{1}\right), \widehat{\psi}\left(a_{3}\right) \in i \mathbb{R}^{+} .
$$

The matrix $R$ has a large $N$ expansion of the form

$$
R(z)=I+\sum_{j=1}^{k} N^{-j} R^{(j)}(z)+\mathcal{O}\left(N^{-k-1}\right), \quad N \rightarrow \infty
$$

for any $k$, where the coefficients $R^{(j)}$ depend on $N$ but remain uniformly bounded for $N$ large. Combining (8.18) with (8.51), we obtain jump relations for the coefficients $R^{(j)}$ : we have

$$
R_{+}^{(k)}(z)=R_{-}^{(k)}(z)+\Delta^{(k)}+\sum_{j=1}^{k-1} R_{-}^{(j)}(z) \Delta^{(k-j)}, \quad z \in \cup_{j=1}^{4} \partial U_{j}
$$

In particular, for $k=1$, this gives

$$
R_{+}^{(1)}(z)-R_{-}^{(1)}(z)=\Delta^{(1)}(z) \quad z \in \partial U_{j}, \quad j=1, \ldots, 4,
$$

where $\Delta^{(1)}$ is given by 8.55 . It is easily seen that $\Delta^{(1)}$ is a meromorphic function in $U_{1}, \ldots, U_{4}$ with double poles at $a_{1}, \ldots, a_{4}$. In addition, by 8.52$), R^{(1)}(z)$ tends to 0 as $z \rightarrow \infty$.

The analyticity of $R^{(1)}$, its jump relations, and the vanishing at infinity define a scalar RH problem for $R^{(1)}$ with a unique solution given by

$$
R^{(1)}(z)=\frac{1}{2 \pi i} \sum_{m=1}^{4} \int_{\partial U_{m}} \frac{\Delta^{(1)}(\lambda)}{\lambda-z} d \lambda .
$$

Since $\Delta^{(1)}(z)$ has double poles at $a_{1}, \ldots, a_{4}$, this yields

$$
R^{(1)}(z)=\sum_{m=1}^{4} \frac{1}{z-a_{m}} \operatorname{Res}_{\lambda=a_{m}} \Delta^{(1)}(\lambda)+\sum_{m=1}^{4} \frac{1}{\left(z-a_{m}\right)^{2}} \underset{\lambda=a_{m}}{\operatorname{Res}}\left(\left(\lambda-a_{m}\right) \Delta^{(1)}(\lambda)\right),
$$

for $z$ outside the disks $U_{m}$. By 8.30$)$ and $(8.28)$, we can write

$$
R^{(1)}\left(z, z_{0}\right)=\sum_{m=1}^{4} \frac{1}{z-a_{m}} \operatorname{Res}_{\lambda=a_{m}} \Delta^{(1)}\left(\lambda, z_{0}\right)+\sum_{m=1}^{4} \frac{1}{\left(z-a_{m}\right)^{2}} \operatorname{Res}_{\lambda=a_{m}}\left(\left(\lambda-a_{m}\right) \Delta^{(1)}\left(\lambda, z_{0}\right)\right),
$$

where we denote

$$
\Delta^{(j)}\left(\lambda, z_{0}\right)= \begin{cases}\frac{1}{\left(-f_{1}(\lambda)\right)^{\frac{3}{2} j}} P^{(\infty)}\left(\lambda, z_{0}\right) \sigma_{3} A_{j} \sigma_{3} P^{(\infty)}\left(\lambda, z_{0}\right)^{-1}, & \lambda \in \partial U_{1}, \\ \frac{1}{f_{2}(\lambda)^{\frac{3}{2} j}} P^{(\infty)}\left(\lambda, z_{0}\right) \mathcal{Q}(\lambda) A_{j} \mathcal{Q}(\lambda)^{-1} P^{(\infty)}\left(\lambda, z_{0}\right)^{-1}, & \lambda \in \partial U_{2}, \\ \frac{1}{\left(-f_{3}(\lambda)\right)^{\frac{3}{2} j}} P^{(\infty)}\left(\lambda, z_{0}\right) \mathcal{Q}(\lambda) \sigma_{3} A_{j} \sigma_{3} \mathcal{Q}(\lambda)^{-1} P^{(\infty)}\left(\lambda, z_{0}\right)^{-1}, & \lambda \in \partial U_{3}, \\ \frac{1}{f_{4}(\lambda)^{\frac{3}{2} j}} P^{(\infty)}\left(\lambda, z_{0}\right) A_{j} P^{(\infty)}\left(\lambda, z_{0}\right)^{-1}, & \lambda \in \partial U_{4} .\end{cases}
$$


Although we will not need this, it is worth noting that a similar procedure applies to the higher order corrections $R^{(j)}(z)$, and that one can recursively find formulas for

$$
R^{(j)}\left(z, z_{0}\right)=P^{\infty}\left(z_{0}\right)^{-1} R^{(j)}(z) P^{\infty}\left(z_{0}\right), \quad j>1 .
$$

\subsection{Evaluation of $(8.33)$}

In this section we will evaluate the quantities appearing at the right hand side of the differential identity (8.33), in terms of other, more canonical quantities, namely the equilibrium measure, and objects from the theory of Riemann surfaces. The final formula is described in Theorem 8.7. We start with the second term.

Lemma 8.4 The following identity is satisfied,

$$
\begin{aligned}
\operatorname{Res}_{z=\infty} \operatorname{Tr}\left(\left.\frac{d P^{\infty}\left(z, z_{0}\right)}{d z}\right|_{z_{0}=z} \sigma_{3} z^{k}\right) & =-2(\log \theta(N \Omega ; B))^{\prime} \operatorname{Res}_{z=\infty^{1}}\left(z^{k} v\left(z^{1}\right) / d z\right) \\
& =-2(\log \theta(N \Omega ; B))^{\prime} \operatorname{Res}_{z=\infty^{1}}\left(z^{k} \frac{1}{\mathcal{A} \sqrt{\mathcal{R}(z)}}\right)
\end{aligned}
$$

where $\mathcal{A}$ is the $\alpha$-period given in (8.36) and where $\mathcal{R}(z)$ is defined by (8.13) and

$$
(\log \theta(N \Omega ; B))^{\prime}:=\left.\frac{d}{d z} \log \theta(z+N \Omega ; B)\right|_{z=0} .
$$

Proof. To prove this formula, we only need to consider the diagonal entries of $P^{\infty}\left(z, z_{0}\right)$. As $z_{0} \rightarrow z$, by 8.42 ,

$$
P_{j j}^{\infty}\left(z, z_{0}\right)=1-\left(z-z_{0}\right)(\log \theta(N \Omega ; B))^{\prime} \frac{v\left(z^{(j)}\right)}{d z}+\mathcal{O}\left(\left(z-z_{0}\right)^{2}, \quad j=1,2,\right.
$$

where $v\left(z^{(2)}\right)=-v\left(z^{(1)}\right)$ is the holomorphic differential defined in 8.37).

It follows that

$$
\operatorname{Tr}\left(\left.\frac{d P^{\infty}\left(z, z_{0}\right)}{d z}\right|_{z_{0}=z} \sigma_{3} z^{k}\right)=-2(\log \theta(N \Omega ; B))^{\prime} z^{k} \frac{v\left(z^{(1)}\right)}{d z},
$$

and this immediately implies 8.66.

The task of evaluating the term

$$
\operatorname{Tr}\left(\left.\frac{d R^{(1)}\left(z, z_{0}\right)}{d z}\right|_{z_{0}=z} \sigma_{3} z^{k}\right)
$$

appearing in 8.33 is considerably more involved. Th goal is to establish an expression in terms of canonical quantities from the theory of Riemann surfaces, so that comparisons to formulae appearing elsewhere in the literature can be validated (or invalidated). Our work relies on two so-called Fay identities, (8.72) and (8.73). In order to explain these identities, we need to introduce some classical quantities and identities in the theory of Riemann surfaces.

The fundamental symmetric bi-differential, introduced in [28] and also called Bergman kernel in some literature, of our Riemann surface, has an explicit expression which is, for

$$
Q, Q_{0} \in \mathcal{S} \backslash\left\{\infty^{1}, \infty^{2},\left(a_{1}, 0\right),\left(a_{2}, 0\right),\left(a_{3}, 0\right),\left(a_{4}, 0\right)\right\},
$$


given by

$$
w\left(Q, Q_{0}\right)=\frac{1}{4}\left(\frac{\gamma(Q)}{\gamma\left(Q_{0}\right)}+\frac{\gamma\left(Q_{0}\right)}{\gamma(Q)}\right)^{2} \frac{d z(Q) d z\left(Q_{0}\right)}{\left(z(Q)-z\left(Q_{0}\right)\right)^{2}}-(\log \theta(\mathbf{0} ; B))^{\prime \prime} v(Q) v\left(Q_{0}\right),
$$

where $\gamma$ has been defined in 8.41). The bi-differential $w\left(Q, Q_{0}\right)$ is analytic for all $Q, Q_{0}$ except for a double pole on the diagonal as $Q \rightarrow Q_{0}$.

We have the well-known relations [28]

$$
\begin{aligned}
& \int_{\beta} w\left(Q, Q_{0}\right)=2 \pi i v(Q), \\
& \int_{Q_{2}}^{Q_{1}} w\left(Q, Q_{0}\right)=\omega_{Q_{1} Q_{2}}(Q),
\end{aligned}
$$

where $\omega_{Q_{1} Q_{2}}(Q)$ is the normalized third kind differential with simple poles at $Q_{1}$ and $Q_{2}$ with residues \pm 1 respectively.

The normalized second kind differential on the elliptic curve $\mathcal{S}$ with poles at $(\infty, \pm \infty)$ of order $k+1$ is given by

$$
\sigma_{k}(z, y)=\frac{P_{k}(z) d z}{y}
$$

where $P_{k}(z)$ is a monic polynomial of degree $k+1$ in $z$ which is determined uniquely by the conditions

$$
\sigma_{k}(z, y)= \pm\left(z^{k-1}+\mathcal{O}\left(z^{-2}\right)\right) d z, \quad \text { as } \quad(z, y) \rightarrow(\infty, \pm \infty)
$$

and

$$
\int_{a_{2}}^{a_{3}} \sigma_{k}=0 .
$$

In order to write $\sigma_{k}(z, y)$ using the fundamental symmetric bi-differential it is sufficient to observe that

$$
\frac{1}{2} \sigma_{k}(z, y)+\frac{1}{2} z^{k-1} d z=-\frac{1}{k} \underset{(\lambda, \eta)=(\infty,+\infty)}{\operatorname{Res}}\left(\lambda^{k} w((z, y),(\lambda, \eta))\right) .
$$

The following important identities connect the product of Szegö kernels to the fundamental symmetric bi-differential. First we have [28, Corollary 2.12]

$$
\begin{aligned}
\widehat{S}\left[\begin{array}{l}
\delta \\
\epsilon
\end{array}\right]\left(Q, Q_{0}\right) \widehat{S}\left[\begin{array}{l}
\delta \\
\epsilon
\end{array}\right]\left(Q_{0}, Q\right) \frac{d z\left(Q_{0}\right) d z(Q)}{\left(z(Q)-z\left(Q_{0}\right)\right)^{2}} \\
=w\left(Q, Q_{0}\right)+\left(\log \theta\left[\begin{array}{l}
\delta \\
\epsilon
\end{array}\right](\mathbf{0} ; B)\right)^{\prime \prime} v(Q) v\left(Q_{0}\right)
\end{aligned}
$$

and secondly [28, Proposition 2.10]

$$
\begin{gathered}
-\widehat{S}\left[\begin{array}{c}
\delta \\
\epsilon
\end{array}\right]\left(Q, P_{0}\right) \widehat{S}\left[\begin{array}{l}
\delta \\
\epsilon
\end{array}\right]\left(Q_{0}, Q\right) \frac{d z(Q)}{\left(z(Q)-z\left(P_{0}\right)\right)\left(z\left(Q_{0}\right)-z(Q)\right)}=\frac{\widehat{S}\left[\begin{array}{l}
\delta \\
\epsilon
\end{array}\right]\left(Q_{0}, P_{0}\right)}{z\left(Q_{0}\right)-z\left(P_{0}\right)} \\
\times\left(\omega_{Q_{0} P_{0}}(Q)+\left[\left(\log \theta\left[\begin{array}{l}
\delta \\
\epsilon
\end{array}\right]\left(\int_{P_{0}}^{Q_{0}} v ; B\right)\right)^{\prime}-\left(\log \theta\left[\begin{array}{c}
\delta \\
\epsilon
\end{array}\right](0 ; B)\right)^{\prime}\right] v(Q)\right),
\end{gathered}
$$


where $\omega_{Q_{0}, P_{0}}(P)$ is the third kind normalized differential with simple poles at the points $P_{0}$ and $Q_{0}$ with residues \pm 1 . In the confluent limit $Q_{0} \rightarrow P_{0}$ the second identity reduces to the first one.

Remark 8.5 In 8.73, we observe that the quantity

$$
\omega_{Q_{0} P_{0}}(Q)+\left[\left(\log \theta\left[\begin{array}{l}
\delta \\
\epsilon
\end{array}\right]\left(\int_{P_{0}}^{Q_{0}} v ; B\right)\right)^{\prime}-\left(\log \theta\left[\begin{array}{l}
\delta \\
\epsilon
\end{array}\right](0 ; B)\right)^{\prime}\right] v(Q)
$$

is a single-valued function of $Q_{0}$ and $P_{0}$. Indeed, defining $d_{Q_{0}}$ as the differentiation with respect to $Q_{0}$, by 8.69 , one has

$$
d_{Q_{0}}\left(\omega_{Q_{0}, P_{0}}(Q)\right)=d_{Q_{0}} \int_{P_{0}}^{Q_{0}} w(Q, \tilde{Q})=w\left(Q, Q_{0}\right),
$$

so that, by (8.68), the integral in $Q_{0}$ of the above identity along the $\alpha$ - and $\beta$-cycles gives

$$
\int_{\beta} d_{Q_{0}}\left(\omega_{Q_{0} P_{0}}(Q)\right)=\int_{\beta} w\left(Q, Q_{0}\right)=2 \pi i v(Q), \quad \int_{\alpha} d_{Q_{0}}\left(\omega_{Q_{0} P_{0}}(Q)\right)=\int_{\alpha} w\left(Q, Q_{0}\right)=0 .
$$

Therefore as $Q_{0} \mapsto Q_{0}+\beta$, we have

$$
\omega_{Q_{0}, P_{0}}(Q) \mapsto \omega_{Q_{0}, P_{0}}(Q)+2 \pi i v(Q),
$$

and as $Q_{0} \mapsto Q_{0}+\alpha$, we have

$$
\omega_{Q_{0}, P_{0}}(Q) \mapsto \omega_{Q_{0}, P_{0}}(Q) .
$$

For the second term in (8.74), as $Q_{0} \mapsto Q_{0}+\alpha$ the logarithm of the $\theta$-function does not change while as $Q_{0} \mapsto Q_{0}+\beta$, by (8.38),

$$
\left(\log \theta\left[\begin{array}{c}
\delta \\
\epsilon
\end{array}\right]\left(\int_{P_{0}}^{Q_{0}} v ; B\right)\right)^{\prime} \mapsto\left(\log \theta\left[\begin{array}{l}
\delta \\
\epsilon
\end{array}\right]\left(\int_{P_{0}}^{Q_{0}} v ; B\right)\right)^{\prime}-2 \pi i .
$$

It follows that 8.74 is a single-valued function of $Q_{0}$. The same consideration applies to the point $P_{0}$.

Introduce the following quantities which contain information about the fundamental bi-differential kernel near the branch points:

$$
\begin{aligned}
& \widehat{w}\left(a_{j}, z\right)=\lim _{\lambda \rightarrow a_{j}} \frac{\left(\lambda-a_{j}\right)^{1 / 2} w\left(\lambda, z^{(1)}\right)}{d \lambda d z} \\
& \widehat{w}^{\prime}\left(a_{j}, z\right)=\lim _{\lambda \rightarrow a_{j}} \frac{\partial}{\partial \lambda}\left[\left(\lambda-a_{j}\right)^{1 / 2}\left(\frac{w\left(\lambda, z^{(1)}\right)}{d \lambda d z}-\frac{1}{2(z-\lambda)^{2}}\right)\right] .
\end{aligned}
$$

Similarly, for the normalized holomorphic differential, we define

$$
\widehat{v}\left(a_{j}\right)=\lim _{\lambda \rightarrow a_{j}}\left(\lambda-a_{j}\right)^{1 / 2} \frac{v(\lambda)}{d \lambda}, \quad \widehat{v}^{\prime}\left(a_{j}\right)=\lim _{\lambda \rightarrow a_{j}} \frac{\partial}{\partial \lambda}\left[\left(\lambda-a_{j}\right)^{1 / 2} \frac{v(\lambda)}{d \lambda}\right] .
$$


Recall also the definition of $\widehat{\psi}\left(a_{m}\right)$ in 8.58). A last quantity we need to introduce is the so-called projective connection $S_{B}\left(a_{j}\right)$ given by

$$
\begin{aligned}
S_{B}\left(a_{j}\right) & =\frac{3}{2} \operatorname{Res}_{\lambda=a_{j}}\left(\frac{d}{d \lambda} \log \prod_{i=1}^{2} \frac{\lambda-a_{2 i}}{\lambda-a_{2 i-1}}\right)^{2}-24(\log \theta(\mathbf{0} ; B))^{\prime \prime} \operatorname{Res}_{\lambda=a_{j}} v(\lambda)^{2} \\
& =3 \sum_{i \neq j}(-1)^{i+j} \frac{1}{a_{j}-a_{i}}-24(\log \theta(\mathbf{0} ; B))^{\prime \prime} \widehat{v}\left(a_{j}\right)^{2} .
\end{aligned}
$$

We are now ready to evaluate the second term at the right hand side of 8.33 .

Theorem 8.6 The following identity is satisfied,

$$
\begin{aligned}
& \frac{1}{2} \operatorname{Res}_{z=\infty}\left(\operatorname{Tr}\left(\left.\frac{d R^{(1)}\left(z, \lambda_{0}\right)}{d z}\right|_{\lambda_{0}=z} \sigma_{3}\right) z^{k}\right) \\
& =\frac{1}{24} \sum_{j=1}^{4} \frac{\operatorname{Res}_{z=\infty^{1}}\left[z^{k} \widehat{w}^{\prime}\left(a_{j}, z\right)\right]}{\left.\widehat{\psi}\left(a_{j}\right)\right)} \\
& \quad-\frac{1}{2} \sum_{j=1}^{4}\left(\frac{1}{4} \frac{\widehat{\psi}^{\prime}\left(a_{j}\right)}{\widehat{\psi}\left(a_{j}\right)}-\frac{1}{6} S_{B}\left(a_{j}\right)-4 \frac{\theta^{\prime \prime}(N \Omega ; B)}{\theta(N \Omega ; B)} \widehat{v}\left(a_{j}\right)^{2}\right) \frac{\operatorname{Res}_{z=\infty^{1}}\left[z^{k} \widehat{w}\left(a_{j}, z\right)\right]}{\widehat{\psi}\left(a_{j}\right)} \\
& +\frac{1}{2}(\log \theta(N \Omega ; B))^{\prime \prime} \operatorname{Res}_{z=\infty}\left[z^{k} \frac{v\left(z^{(1)}\right)}{d z}\right] \sum_{j=1}^{4} \frac{\frac{1}{6} S_{B}\left(a_{j}\right) \widehat{v}\left(a_{j}\right)+\frac{1}{12} \widehat{v}^{\prime}\left(a_{j}\right)-\frac{1}{4} \frac{\widehat{\psi}\left(a_{j}\right)}{\widehat{\psi}\left(a_{j}\right)} \widehat{v}\left(a_{j}\right)}{\widehat{\psi}\left(a_{j}\right)} \\
& \quad+\frac{2}{3}\left(\frac{(\theta(N \Omega ; B))^{\prime \prime \prime}}{\theta(N \Omega ; B)}\right)^{\prime} \operatorname{Res}_{z=\infty}\left[z^{k} \frac{v\left(z^{(1)}\right)}{d z}\right] \sum_{j=1}^{4} \frac{\widehat{v}\left(a_{j}\right)^{3}}{\widehat{\psi}\left(a_{j}\right)} . \quad(8.79)
\end{aligned}
$$

Proof. Observe by 8.64 that

$$
\begin{aligned}
& \frac{1}{2} \operatorname{Res}_{z=\infty} \operatorname{Tr}\left(\left.\frac{d R^{(1)}\left(z, z_{0}\right)}{d z}\right|_{z_{0}=z} z^{k} \sigma_{3}\right) \\
& \quad=-\frac{1}{2} \operatorname{Res}_{z=\infty}\left(\sum_{j=1}^{4} \frac{z^{k}}{\left(z-a_{j}\right)^{2}} \underset{\lambda=a_{j}}{\operatorname{Res}}\left[\left(1+2 \frac{\lambda-a_{j}}{z-a_{j}}\right) \operatorname{Tr}\left(\Delta^{(1)}(\lambda, z) \sigma_{3}\right)\right)\right] .
\end{aligned}
$$

From the definitions of $\Delta^{(1)}$ and $A_{1}$ in 8.54 - 8.55, one has

$$
\operatorname{Tr}\left(\Delta^{(1)}(\lambda, z) \sigma_{3}\right)=\Sigma_{1}(\lambda, z)+\Sigma_{2}(\lambda, z)
$$

with

$$
\begin{aligned}
\Sigma_{1}(\lambda, z)=\frac{1}{48} \frac{1}{\left((-1)^{j} f_{j}(\lambda)\right)^{\frac{3}{2}}} & \left(P_{11}^{\infty}(\lambda, z) P_{11}^{\infty}(z, \lambda)-P_{12}^{\infty}(\lambda, z) P_{21}^{\infty}(z, \lambda)\right. \\
& \left.+P_{22}^{\infty}(\lambda, z) P_{22}^{\infty}(z, \lambda)-P_{21}^{\infty}(\lambda, z) P_{12}^{\infty}(z, \lambda)\right),
\end{aligned}
$$


for $z$ near $a_{j}$, and for $\operatorname{Im} \lambda \geq 0$, we have

$$
\Sigma_{2}(\lambda, z)=\left\{\begin{array}{l}
-\frac{i}{4} \frac{1}{\left(-f_{1}(\lambda)\right)^{\frac{3}{2}}}\left(P_{12}^{\infty}(\lambda, z) P_{11}^{\infty}(z, \lambda)-P_{21}^{\infty}(\lambda, z) P_{22}^{\infty}(z, \lambda)\right), \\
\frac{i}{4} \frac{1}{f_{2}(\lambda)^{\frac{3}{2}}}\left(P_{12}^{\infty}(\lambda, z) P_{11}^{\infty}(z, \lambda) e^{-2 \pi i N \Omega}-P_{21}^{\infty}(\lambda, z) P_{22}^{\infty}(z, \lambda) e^{2 \pi i N \Omega}\right), \\
-\frac{i}{4} \frac{1}{\left(-f_{3}(\lambda)\right)^{\frac{3}{2}}}\left(P_{12}^{\infty}(\lambda, z) P_{11}^{\infty}(z, \lambda) e^{-2 \pi i N \Omega}-P_{21}^{\infty}(\lambda, z) P_{22}^{\infty}(z, \lambda) e^{2 \pi i N \Omega}\right), \\
\frac{i}{4} \frac{1}{f_{4}(\lambda)^{\frac{3}{2}}}\left(P_{12}^{\infty}(\lambda, z) P_{11}^{\infty}(z, \lambda)-P_{21}^{\infty}(\lambda, z) P_{22}^{\infty}(z, \lambda)\right),
\end{array}\right.
$$

where we used the fact that $P^{\infty}(\lambda, z)^{-1}=P^{\infty}(z, \lambda)$, and where the first line is valid for $\lambda$ near $a_{1}$, the second near $a_{2}$, etc.

For each of the matrix products at the right hand side of 8.82 , the identity 8.72 which connects the Szegö kernel and the fundamental bi-differential. For instance,

$$
\begin{aligned}
P_{k j}^{\infty}(\lambda, z) & P_{j k}^{\infty}(z, \lambda) \\
& =\left(w\left(\lambda^{(j)}, z^{(k)}\right)+(\log \theta(N \Omega))^{\prime \prime} v\left(\lambda^{(j)}\right) v\left(z^{(k)}\right)\right) \frac{(\lambda-z)^{2}}{d \lambda d z}, \quad j, k=1,2 .
\end{aligned}
$$

Using the expression of the fundamental bi-differential in (8.67), by (8.75), one obtains expansions of the following form as $\lambda \rightarrow a_{j}$ :

$$
\begin{aligned}
& \frac{w\left(\lambda^{(1)}, z\right)}{d \lambda d z}=\frac{\widehat{w}\left(a_{j}, z\right)}{\left(\lambda-a_{j}\right)^{1 / 2}}+\frac{1}{2\left(z-a_{j}\right)^{2}}+\widehat{w}^{\prime}\left(a_{j}, z\right)\left(\lambda-a_{j}\right)^{1 / 2}+\mathcal{O}\left(\lambda-a_{j}\right), \\
& \frac{w\left(\lambda^{(2)}, z\right)}{d \lambda d z}=-\frac{\widehat{w}\left(a_{j}, z\right)}{\left(\lambda-a_{j}\right)^{1 / 2}}+\frac{1}{2\left(z-a_{j}\right)^{2}}-\widehat{w}^{\prime}\left(a_{j}, z\right)\left(\lambda-a_{j}\right)^{1 / 2}+\mathcal{O}\left(\lambda-a_{j}\right),
\end{aligned}
$$

and

$$
\frac{v\left(\lambda^{(1)}\right)}{d \lambda}=-\frac{v\left(\lambda^{(2)}\right)}{d \lambda}=\frac{\widehat{v}\left(a_{j}\right)}{\left(\lambda-a_{j}\right)^{1 / 2}}+\widehat{v}^{\prime}\left(a_{j}\right)\left(\lambda-a_{j}\right)^{1 / 2}+\mathcal{O}\left(\lambda-a_{j}\right) .
$$

Combining (8.57), (8.81), (8.84), and 8.85), we obtain the following relation for the residues of $\Sigma_{1}(\lambda, z)$ defined in $(8.82)$,

$$
\begin{array}{r}
-\sum_{j=1}^{4} \frac{1}{\left(z-a_{j}\right)^{2}} \operatorname{Res}_{\lambda=a_{j}} \Sigma_{1}(\lambda, z)-2 \sum_{j=1}^{4} \frac{1}{\left(z-a_{j}\right)^{3}} \operatorname{Res}_{\lambda=a_{j}}\left(\left(\lambda-a_{j}\right) \Sigma_{1}(\lambda, z)\right) \\
=-\frac{1}{12} \frac{1}{\widehat{\psi}\left(a_{j}\right)}\left[\widehat{w}^{\prime}\left(a_{j}, z^{(1)}\right)+(\log \theta(N \Omega))^{\prime \prime} \widehat{v}^{\prime}\left(a_{j}\right) \frac{v\left(z^{(1)}\right)}{d z}\right] \\
+\frac{1}{20} \frac{\widehat{\psi}^{\prime}\left(a_{j}\right)}{\widehat{\psi}\left(a_{j}\right)^{2}}\left[\widehat{w}\left(a_{j}, z^{(1)}\right)+(\log \theta(N \Omega))^{\prime \prime} \widehat{v}\left(a_{j}\right) \frac{v\left(z^{(1)}\right)}{d z}\right] .
\end{array}
$$

In order to evaluate the residues of products of the form $P_{12}^{\infty}(\lambda, z) P_{11}^{\infty}(z, \lambda)$ in 
$\Sigma_{2}(\lambda, z)$ defined in 8.83 , we use 8.73 which gives

$$
\begin{aligned}
& P_{12}^{\infty}(\lambda, z) P_{11}^{\infty}(z, \lambda)=\lim _{\eta \rightarrow \lambda} \frac{\widehat{S}\left[{ }_{-N \Omega}^{0}\right]\left(\eta^{(2)}, \lambda^{(1)}\right)}{\eta-\lambda} \frac{(\lambda-z)^{2}}{d z} \times \\
& \quad\left[v\left(z^{(1)}\right)\left(\left(\log \theta\left(\int_{\lambda^{(1)}}^{\lambda^{(2)}} v-N \Omega\right)\right)^{\prime}-(\log \theta(-N \Omega))^{\prime}\right)+\omega_{\lambda^{(2)} \lambda^{(1)}}\left(z^{(1)}\right)\right],
\end{aligned}
$$

and similarly

$$
\begin{aligned}
& P_{21}^{\infty}(\lambda, z) P_{22}^{\infty}(z, \lambda)=\lim _{\eta \rightarrow \lambda} \frac{\widehat{S}\left[\begin{array}{l}
0 \\
-N \Omega
\end{array}\right]\left(\eta^{(1)}, \lambda^{(2)}\right)}{\eta-\lambda} \frac{(\lambda-z)^{2}}{d z} \times \\
& {\left[v\left(z^{(2)}\right)\left(\left(\log \theta\left(\int_{\lambda^{(2)}}^{\lambda^{(1)}} v-N \Omega\right)\right)^{\prime}-(\log \theta(-N \Omega))^{\prime}\right)+\omega_{\lambda^{(1)} \lambda^{(2)}}\left(z^{(2)}\right)\right],}
\end{aligned}
$$

where $\omega_{\lambda^{(1)} \lambda^{(2)}}\left(z^{(j)}\right), j=1,2$, stands as before for the normalized third kind differential, in this case with poles at two conjugate points $\lambda^{(1)}$ and $\lambda^{(2)}$. Next, using (8.69), we have the following identities for a third kind differential with poles at two conjugate points $\lambda^{(2)}$ and $\lambda^{(1)}$,

$$
\omega_{\lambda^{(1)} \lambda^{(2)}}(P)=-\omega_{\lambda^{(2)} \lambda^{(1)}}(P), \quad \omega_{\lambda^{(2)} \lambda^{(1)}}\left(z^{(1)}\right)=\omega_{\lambda^{(1)} \lambda^{(2)}}\left(z^{(2)}\right),
$$

and we have the following expansion as $\lambda \rightarrow a_{j}$,

$$
\omega_{\lambda^{(1)}, \lambda^{(2)}}\left(z^{(1)}\right)=4 \widehat{w}\left(a_{j}, z^{(1)}\right)\left(\lambda-a_{j}\right)^{1 / 2}+\frac{4}{3} \widehat{w}^{\prime}\left(a_{j}, z^{(1)}\right)\left(\lambda-a_{j}\right)^{3 / 2}+\mathcal{O}\left(\left(\lambda-a_{j}\right)^{5 / 2}\right) .
$$

In order to proceed, we need to evaluate the expansions in $\lambda$ of the quantities (8.87) and 8.88 as $\lambda \rightarrow a_{j}, j=1,2,3,4$. First we observe that

$$
\lim _{\eta \rightarrow \lambda} \frac{\widehat{S}\left[\begin{array}{c}
0 \\
-N \Omega
\end{array}\right]\left(\eta^{(1)}, \lambda^{(2)}\right)}{\eta-\lambda}=\frac{i}{4} \frac{\theta(0 ; B)}{\theta(N \Omega ; B)} \frac{\theta\left(\int_{\lambda^{(2)}}^{\lambda^{(1)}} v-N \Omega ; B\right)}{\theta\left(\int_{\lambda^{(2)}}^{\lambda^{(1)}} v ; B\right)} \sum_{k=1}^{4} \frac{(-1)^{k}}{\lambda-a_{k}},
$$

and

$$
\lim _{\eta \rightarrow \lambda} \frac{\widehat{S}\left[\begin{array}{c}
0 \\
-N \Omega
\end{array}\right]\left(\eta^{(2)}, \lambda^{(1)}\right)}{\eta-\lambda}=-\frac{i}{4} \frac{\theta(0 ; B)}{\theta(N \Omega ; B)} \frac{\theta\left(\int_{\lambda^{(2)}}^{\lambda^{(1)}} v+N \Omega ; B\right)}{\theta\left(\int_{\lambda^{(2)}}^{\lambda^{(1)}} v ; B\right)} \sum_{k=1}^{4} \frac{(-1)^{k}}{\lambda-a_{k}}
$$

as follows from the definition 8.40 and the symmetry $\theta(z ; B)=\theta(-z ; B)$. Therefore using the parity property of $\theta$-function, 8.89, 8.91) and (8.92), the identities 8.87) 
and 8.88 take the form

$$
\begin{gathered}
P_{12}^{\infty}(\lambda, z) P_{11}^{\infty}(z, \lambda)=-\frac{i}{4} \frac{\theta(0 ; B)}{\theta(N \Omega ; B)} \frac{\theta\left(\int_{\lambda^{(2)}}^{\lambda^{(1)}} v+N \Omega ; B\right)}{\theta\left(\int_{\lambda^{(2)}}^{\lambda^{(1)}} v ; B\right)} \sum_{k=1}^{4} \frac{(-1)^{k}}{\lambda-a_{k}} \frac{(\lambda-z)^{2}}{d z} \times \\
{\left[-v\left(z^{(1)}\right)\left(\left(\log \theta\left(\int_{\lambda^{(2)}}^{\lambda^{(1)}} v+N \Omega\right)\right)^{\prime}-(\log \theta(N \Omega))^{\prime}\right)-\omega_{\left.\lambda^{(1)} \lambda^{(2)}\left(z^{(1)}\right)\right],}\right.}
\end{gathered}
$$

and similarly

$$
\begin{gathered}
P_{21}^{\infty}(\lambda, z) P_{22}^{\infty}(z, \lambda)=\frac{i}{4} \frac{\theta(0 ; B)}{\theta(N \Omega ; B)} \frac{\theta\left(\int_{\lambda^{(2)}}^{\lambda^{(1)}} v-N \Omega ; B\right)}{\theta\left(\int_{\lambda^{(2)}}^{\lambda^{(1)}} v ; B\right)} \sum_{k=1}^{4} \frac{(-1)^{k}}{\lambda-a_{k}} \frac{(\lambda-z)^{2}}{d z} \times \\
{\left[-v\left(z^{(1)}\right)\left(\left(\log \theta\left(\int_{\lambda^{(2)}}^{\lambda^{(1)}} v-N \Omega\right)\right)^{\prime}-(\log \theta(-N \Omega))^{\prime}\right)-\omega_{\lambda^{(1)} \lambda^{(2)}}\left(z^{(1)}\right)\right] .}
\end{gathered}
$$

For the integrals between $\lambda^{(2)}$ and $\lambda^{(1)}$, we follow a path which goes through $a_{4}$. Consequently, for $\operatorname{Im} \lambda \geq 0$, one has

$$
\int_{\lambda^{(2)}}^{\lambda^{(1)}} v=\left\{\begin{array}{l}
2 \int_{a_{4}}^{\lambda^{(1)}} v \\
2 \int_{a_{3}}^{\lambda^{(1)}} v-B \\
2 \int_{a_{2}}^{\lambda^{(1)}} v-B-1 \\
2 \int_{a_{1}}^{\lambda^{(1)}} v-1
\end{array}\right.
$$

and as $\lambda \rightarrow a_{j}, j=1,2,3,4$, we obtain

$$
2 \int_{a_{j}}^{\lambda^{(1)}} v=4 \widehat{v}\left(a_{j}\right)\left(\lambda-a_{j}\right)^{1 / 2}+\frac{4}{3} \widehat{v}^{\prime}\left(a_{j}\right)\left(\lambda-a_{j}\right)^{\frac{3}{2}}+\mathcal{O}\left(\left(\lambda-a_{j}\right)^{\frac{5}{2}}\right),
$$

with $\widehat{v}\left(a_{j}\right)$ defined in 8.76$)$. Using (8.38), 8.91), 8.92), 8.95), 8.96), and the fact 
that $\theta(z ; B)$ is an even function of $z$, we obtain, as $\lambda \rightarrow a_{j}$ from the upper half plane,

$$
\begin{aligned}
& \frac{i}{4} \frac{\theta(0 ; B)}{\theta(N \Omega ; B)} \frac{\theta\left(\int_{\lambda^{(2)}}^{\lambda^{(1)}} v \pm N \Omega ; B\right)}{\theta\left(\int_{\lambda^{(2)}}^{\lambda^{(1)}} v ; B\right)} \sum_{k=1}^{4} \frac{(-1)^{k}}{\lambda-a_{k}} \\
& =\frac{i(-1)^{j}}{4}\left(\frac{1}{\lambda-a_{j}} \pm 4 \widehat{v}\left(a_{j}\right) \frac{\theta^{\prime}}{\theta}(N \Omega ; B)\left(\lambda-a_{j}\right)^{-1 / 2}+\sum_{k \neq j} \frac{(-1)^{k+j}}{a_{j}-a_{k}}\right. \\
& \left.\quad+8 \widehat{v}\left(a_{j}\right)^{2}\left(\frac{\theta^{\prime \prime}}{\theta}(N \Omega ; B)-\frac{\theta^{\prime \prime}}{\theta}(0 ; B)\right)+o(1)\right) \times \begin{cases}1 & j=1,4, \\
e^{ \pm 2 \pi i N \Omega} & j=2,3 .\end{cases}
\end{aligned}
$$

Using the definition of the projective connection given in (8.77), we can rewrite the above expansion as

$$
\begin{gathered}
\frac{i}{4} \frac{\theta(0 ; B)}{\theta(N \Omega ; B)} \frac{\theta\left(\int_{\lambda^{(2)}}^{\lambda^{(1)}} v \pm N \Omega ; B\right)}{\theta\left(\int_{\lambda^{(2)}}^{\lambda^{(1)}} v ; B\right)} \sum_{k=1}^{4} \frac{(-1)^{k}}{\lambda-a_{k}} \\
=\frac{i(-1)^{j}}{4}\left(\frac{1}{\lambda-a_{j}} \pm 4 \widehat{v}\left(a_{j}\right) \frac{\theta^{\prime}}{\theta}(N \Omega ; B)\left(\lambda-a_{j}\right)^{-1 / 2}+\frac{1}{3} S_{B}\left(a_{j}\right)\right. \\
\left.+8 \widehat{v}\left(a_{j}\right)^{2} \frac{\theta^{\prime \prime}}{\theta}(N \Omega ; B)\right) \times \begin{cases}1 & j=1,4, \\
e^{ \pm 2 \pi i N \Omega} & j=2,3 .\end{cases}
\end{gathered}
$$

Combining (8.57), 8.83), (8.90), 8.96), 8.93), 8.94), and (8.98), one arrives with the help of Maple to the relation

$$
\begin{aligned}
& -\sum_{j=1}^{4} \frac{\operatorname{Res}_{\lambda=a_{j}} \Sigma_{2}(\lambda, z) d z}{\left(z-a_{j}\right)^{2}}-2 \sum_{j=1}^{4} \frac{\operatorname{Res}_{\lambda=a_{j}}\left(\left(\lambda-a_{j}\right) \Sigma_{2}(\lambda, z) d z\right)}{\left(z-a_{j}\right)^{3}} \\
& =\sum_{j=1}^{4} \frac{\widehat{w}^{\prime}\left(a_{j}, z^{(1)}\right)+(\log \theta(N \Omega))^{\prime \prime} \widehat{v}^{\prime}\left(a_{j}\right) v\left(z^{(1)}\right)}{6 \widehat{\psi}\left(a_{j}\right)} \\
& +\sum_{j=1}^{4} \frac{\left(\frac{1}{6} S_{B}\left(a_{j}\right)+4 \frac{\theta^{\prime \prime}(N \Omega)}{\theta(N \Omega)} \widehat{v}\left(a_{j}\right)^{2}-\frac{3}{10} \frac{\widehat{\psi}^{\prime}\left(a_{j}\right)}{\widehat{\psi}\left(a_{j}\right)}\right)}{\widehat{\psi}\left(a_{j}\right)}\left(\widehat{w}\left(a_{j}, z^{(1)}\right)+(\log \theta(N \Omega))^{\prime \prime} \widehat{v}\left(a_{j}\right) v\left(z^{(1)}\right)\right) \\
& +\frac{4}{3} \sum_{j=1}^{4} \frac{\widehat{v}\left(a_{j}\right)^{3} v\left(z^{(1)}\right)}{\widehat{\psi}\left(a_{j}\right)}\left((\log \theta(N \Omega))^{\prime \prime \prime \prime}+3(\log \theta(N \Omega))^{\prime \prime \prime}(\log \theta(N \Omega))^{\prime}\right) . \quad(8.99)
\end{aligned}
$$

Summing 8.86 and 8.99 and multiplying by $1 / 2$ we obtain 8.79 and the theorem is proven.

Combining the heat equation 8.39 , Lemma 8.2, Lemma 8.4, and Theorem 8.6, we get to the following identity. 
Theorem 8.7 For a two-cut regular potential $V_{\vec{t}}$, the logarithmic derivative of the partition function with respect to the parameters $t_{k}$, has the following asymptotic expansion as $N \rightarrow \infty$,

$$
\begin{aligned}
& \frac{\partial}{\partial t_{k}} \log Z_{N}\left(V_{\vec{t}}\right)=-N^{2} \frac{\partial}{\partial t_{k}} F_{0}-N\left(\log (\theta(N \Omega ; B))^{\prime} \operatorname{Res}_{z=\infty}\left[z^{k} v\left(z^{(1)}\right)\right]\right. \\
& \quad+\frac{1}{24} \sum_{j=1}^{4} \frac{\operatorname{Res}_{z=\infty^{1}}\left[z^{k} \widehat{w}^{\prime}\left(a_{j}, z\right)\right]}{\widehat{\psi}\left(a_{j}\right)} \\
& \quad-\frac{1}{2} \sum_{j=1}^{4}\left(\frac{1}{4} \frac{\widehat{\psi}\left(a_{j}\right)}{\widehat{\psi}\left(a_{j}\right)}-\frac{1}{6} S_{B}\left(a_{j}\right)-16 \pi i \frac{\frac{\partial}{\partial B} \theta(N \Omega ; B)}{\theta(N \Omega ; B)} \widehat{v}\left(a_{j}\right)^{2}\right) \frac{\operatorname{Res}_{z=\infty^{1}}\left[z^{k} \widehat{w}\left(a_{j}, z\right)\right]}{\widehat{\psi}\left(a_{j}\right)} \\
& +\left(\frac{1}{6}\left(\frac{(\theta(N \Omega ; B))^{\prime \prime \prime}}{\theta(N \Omega ; B)}\right)^{\prime} F_{0}^{(3)}-(\log \theta(N \Omega ; B))^{\prime \prime} F_{1}^{(1)}\right) \operatorname{Res}_{z=\infty}\left[z^{k} v\left(z^{(1)}\right)\right]+\mathcal{O}\left(N^{-1}\right),
\end{aligned}
$$

where

$$
F_{1}^{(1)}:=\frac{1}{2} \sum_{j=1}^{4} \frac{\widehat{v}\left(a_{j}\right)}{\widehat{\psi}\left(a_{j}\right)}\left(\frac{1}{4} \frac{\widehat{\psi}^{\prime}\left(a_{j}\right)}{\widehat{\psi}\left(a_{j}\right)}-\frac{S_{B}\left(a_{j}\right)}{6}-\frac{\widehat{v}^{\prime}\left(a_{j}\right)}{12 \widehat{v}\left(a_{j}\right)}\right),
$$

and

$$
F_{0}^{(3)}:=4 \sum_{j=1}^{4} \frac{\widehat{v}\left(a_{j}\right)^{3}}{\widehat{\psi}\left(a_{j}\right)},
$$

and all the other quantities are defined in the statement of Theorem 8.6.

We remark that the definition of $F_{1}^{(1)}$ and $F_{0}^{(3)}$ given by Eynard in [26] (see also [27]) coincides with our definition up to multiplicative factors.

\subsection{Calculating antiderivatives}

In this section, we will express the terms in 8.100 as derivatives. We start with the projective connection $S_{B}\left(a_{j}\right)$.

Lemma 8.8 [38, 37] Let $\Delta(\boldsymbol{a})=\prod_{1 \leq j<k \leq 4}\left(a_{k}-a_{j}\right)$ be the Vandermonde determinant of the branch points of the surface $\mathcal{S}$ and $\mathcal{A}$ the $\alpha$-period of the non-normalized holomorphic differential as defined in (8.36). Then the following relation is satisfied,

$$
\frac{\partial}{\partial a_{j}} \log \left(\mathcal{A}^{12} \Delta(\boldsymbol{a})^{3}\right)=-S_{B}\left(a_{j}\right), \quad j=1,2,3,4,
$$

where $S_{B}\left(a_{j}\right)$ at the branch point has been defined (8.77).

This identity was derived in [38] for more general surfaces than our elliptic surface, namely for generic non-singular hyper-elliptic Riemann surfaces. Next we are going to evaluate the derivatives with respect to the times $t_{k}$ of all the relevant quantities appearing in 8.66 ) and (8.79). Some of the identities below have already appeared in the literature, see for example [32, 31, 41, 43], but for clarity we are going to prove each of them with our notation. 
Lemma 8.9 The following identities are satisfied:

$$
\begin{aligned}
& \frac{\partial}{\partial t_{k}} \Omega=-\operatorname{Res}_{\lambda=\infty^{1}}\left[\lambda^{k} v(\lambda)\right], \\
& \frac{\partial a_{m}}{\partial t_{k}}=-\frac{k \widehat{\sigma}_{k}\left(a_{m}\right)}{\widehat{\psi}\left(a_{m}\right)}=2 \frac{\operatorname{Res}_{\lambda=\infty^{1}}\left[\lambda^{k} \widehat{w}\left(\lambda, a_{m}\right)\right]}{\widehat{\psi}\left(a_{m}\right)} \\
& \frac{\partial B}{\partial t_{k}}=\sum_{m=1}^{4} \frac{\partial B}{\partial a_{m}} \frac{\partial a_{m}}{\partial t_{k}}=8 \pi i \sum_{m} \widehat{v}\left(a_{m}\right)^{2} \frac{\operatorname{Res}_{\lambda=\infty^{1}}\left[\lambda^{k} \widehat{w}\left(\lambda^{(1)}, a_{m}\right)\right]}{\widehat{\psi}\left(a_{m}\right)}, \\
& \frac{\partial \widehat{\psi}\left(a_{m}\right)}{\partial t_{k}}=3 \frac{\widehat{\psi}^{\prime}\left(a_{m}\right)}{\widehat{\psi}\left(a_{m}\right)} \operatorname{Res}_{\lambda=\infty^{1}}\left[\lambda^{k} \widehat{w}\left(\lambda, a_{m}\right)\right]-\operatorname{Res}_{\lambda=\infty^{1}}\left[\lambda^{k} \widehat{w}^{\prime}\left(a_{m}, \lambda\right)\right],
\end{aligned}
$$

where

$$
\widehat{\sigma}_{k}\left(a_{m}\right):=\left.\frac{\sqrt{\lambda-a_{m}} \sigma_{k}(\lambda)}{d \lambda}\right|_{\lambda=a_{m}}
$$

with $\sigma_{k}$ the meromorphic differential defined in (8.70), $\widehat{w}\left(a_{j}, z\right)$ and $\widehat{w}^{\prime}\left(a_{j}, z\right)$ defined in (8.75).

Proof. Recall from (8.11) that the density $\psi(\lambda)$ of the equilibrium measure takes the form

$$
\psi(\lambda)=\frac{h(\lambda)}{\pi i} \mathcal{R}(\lambda)_{+}^{1 / 2}, \quad x \in\left[a_{1}, a_{2}\right] \cup\left[a_{3}, a_{4}\right],
$$

for a polynomial $h(\lambda)$. The quantity

$$
h(\lambda) \mathcal{R}(\lambda)^{1 / 2} d \lambda
$$

can be seen as a one-form on the Riemann surface, which

(1) has simple zeros at the branch points $\left(a_{i}, 0\right) i=1 \ldots, 4$,

(2) has a pole at $\infty^{1,2}=(\infty, \pm \infty)$ with asymptotic behaviour

$$
h(\lambda) \mathcal{R}(\lambda)^{1 / 2} d \lambda= \pm\left(\frac{1}{2} V_{\vec{t}}^{\prime}(\lambda)-\frac{1}{\lambda}-\mathcal{O}\left(\lambda^{-2}\right)\right) d \lambda
$$

because of the moments conditions 8.15,

(3) is normalized to zero on the $\alpha$-cycle because of 8.16 .

By 8.1), it follows that 8.109 can be written also in the form

$$
h(\lambda) \sqrt{\mathcal{R}(\lambda)} d \lambda=\frac{1}{2} \sum_{j=1}^{2 d} j t_{j} \sigma_{j}+2 \sigma_{4}-4 \sigma_{2}-\sigma_{0},
$$

where $\sigma_{j}=\sigma_{j}(\lambda, y)$ are the second kind differentials defined in 8.70 , and $\sigma_{0}$ is a differential of the third kind uniquely determined by the conditions of having simple poles at $\infty^{1,2}$ with residue \pm 1 and of being normalized to zero on the $\alpha$-cycles. It should be noted that the right hand side of (8.111) depends on the endpoints $a_{1}, \ldots, a_{4}$ through the normalization. 
The one-form on the left hand side of (8.111) is built in such a way that it satisfies conditions (1), but conditions (2) and (3) are imposed by (8.15) and (8.16). The oneform on the right hand side of (8.111) is built in such a way that it satisfies conditions (2) and (3), while the condition (1) has to be imposed:

$$
\left(\sum_{j=1}^{2 d} j t_{j} \sigma_{j}(\lambda, y)+2 \sigma_{4}-4 \sigma_{2}-2 \sigma_{0}(\lambda, y)\right)_{(\lambda, y)=\left(a_{p}, 0\right)}=0, \quad p=1, \ldots, 4 .
$$

These 4 equations determine the endpoints $a_{1}<a_{2}<a_{3}<a_{4}$ of the support of the equilibrium measure and are equivalent to 8.15 and (8.16). Now we take the derivatives with respect to times $t_{k}$,

$$
\begin{aligned}
& \frac{\partial}{\partial t_{k}}\left(\sum_{j=1}^{2 d} j t_{j} \sigma_{j}+2 \sigma_{4}-4 \sigma_{2}-2 \sigma_{0}\right) \\
& =k \sigma_{k}+\sum_{p}\left(\sum_{j=1}^{2 d} j t_{j} \frac{\partial}{\partial a_{p}} \sigma_{j}+2 \frac{\partial}{\partial a_{p}} \sigma_{4}-4 \frac{\partial}{\partial a_{p}} \sigma_{2}-2 \frac{\partial}{\partial a_{p}} \sigma_{0}\right) \frac{\partial a_{p}}{\partial t_{k}} \\
& =k \sigma_{k},
\end{aligned}
$$

where in the last identity we use the fact that

$$
\sum_{j=1}^{2 d} j t_{j} \frac{\partial}{\partial a_{p}} \sigma_{j}+2 \frac{\partial}{\partial a_{p}} \sigma_{4}-4 \frac{\partial}{\partial a_{p}} \sigma_{2}-2 \frac{\partial}{\partial a_{p}} \sigma_{0}
$$

is a normalized one-form, regular at $\infty^{1,2}$ because of 8.110 , and which can only have a singularity at the branch point $\left(a_{p}, 0\right)$. However, by (8.112), this differential is regular at $\left(a_{p}, 0\right), p=1,2,3,4$. Therefore, $\sum_{j=1}^{2 d} j t_{j} \frac{\partial}{\partial a_{p}} \sigma_{j}+2 \frac{\partial}{\partial a_{p}} \sigma_{4}-4 \frac{\partial}{\partial a_{p}} \sigma_{2}-2 \frac{\partial}{\partial a_{p}} \sigma_{0}$ is a normalized holomorphic one form and by Riemann's theorem it is identically zero. Such an argument was first used in [40]. Therefore we can conclude that

$$
\frac{\partial}{\partial t_{k}} \frac{h(\lambda)}{\pi i} \mathcal{R}(\lambda)^{1 / 2} d \lambda=\frac{\partial}{\partial t_{k}} \operatorname{Re} \frac{1}{\pi i}\left(\frac{1}{2} \sum_{j=1}^{2 d} j t_{j} \sigma_{j}+2 \sigma_{4}-4 \sigma_{2}-\sigma_{0}\right)=\operatorname{Re}\left(\frac{k \sigma_{k}}{2 \pi i}\right) .
$$

Hence,

$$
\frac{\partial}{\partial t_{k}} \Omega=\frac{1}{2 \pi i} \frac{\partial}{\partial t_{k}} \int_{\beta} h(\lambda) \mathcal{R}(\lambda)^{1 / 2} d \lambda=\frac{k}{4 \pi i} \int_{\beta} \sigma_{k} .
$$

By Riemann's bilinear relations [28, we have

$$
\int_{\beta} \sigma_{k}=-\frac{4 \pi i}{k} \operatorname{Res}_{(\lambda, y)=\infty^{1}}\left[\lambda^{k} v(\lambda, y)\right]
$$

so that

$$
\frac{\partial}{\partial t_{k}} \Omega=-\operatorname{Res}_{(\lambda, y)=\infty^{1}}\left[\lambda^{k} v(\lambda, y)\right]
$$

which proves 8.104. 
In order to prove 8.105 we observe that, for general values of $a_{1}, \ldots, a_{4}$, we can write

$$
\frac{1}{2} \sum_{j=1}^{2 d} j t_{j} \sigma_{j}+2 \sigma_{4}-4 \sigma_{2}-\sigma_{0}=\frac{Z(\lambda)}{\mathcal{R}(\lambda)^{1 / 2}} d \lambda,
$$

where $Z(\lambda)$ is a polynomial of degree $2 d+1$. We can write the polynomial $Z(\lambda)$ in the form

$$
Z(\lambda)=h(\lambda) \prod_{j=1}^{4}\left(\lambda-a_{j}\right)+Z_{0}(\lambda)
$$

where $Z_{0}(\lambda)$ is a polynomial of degree at most 3 . By (8.111), $Z_{0}(\lambda)$ is identically zero if $a_{1}, \ldots, a_{4}$ are such that 8.112 holds. By 8.113 , one has

$$
\begin{aligned}
\frac{\partial}{\partial t_{k}}\left(\left(\lambda-a_{m}\right)^{1 / 2} \frac{Z(\lambda) d \lambda}{\mathcal{R}(\lambda)^{1 / 2}}\right) & =-\frac{1}{2\left(\lambda-a_{m}\right)^{1 / 2}} \frac{Z(\lambda) d \lambda}{\mathcal{R}(\lambda)^{1 / 2}} \frac{\partial a_{m}}{\partial t_{k}}+\left(\lambda-a_{m}\right)^{1 / 2} \frac{\partial}{\partial t_{k}} \frac{Z(\lambda) d \lambda}{\mathcal{R}(\lambda)^{1 / 2}} \\
= & -\frac{1}{2\left(\lambda-a_{m}\right)^{1 / 2}} \frac{Z(\lambda) d \lambda}{\mathcal{R}(\lambda)^{1 / 2}} \frac{\partial a_{m}}{\partial t_{k}}+\frac{k}{2}\left(\lambda-a_{m}\right)^{1 / 2} \sigma_{k}(\lambda) .
\end{aligned}
$$

This holds for any value of $\lambda$. Keeping in mind that the left hand side has a zero at $\lambda=a_{m}$, we can evaluate at $\lambda=a_{m}$ and obtain

$$
-h\left(a_{m}\right) \sqrt{\prod_{j \neq m}\left(a_{m}-a_{j}\right)} \frac{\partial a_{m}}{\partial t_{k}}=-\left(\frac{1}{2 \sqrt{\lambda-a_{m}}} \frac{Z(\lambda) d \lambda}{\sqrt{\mathcal{R}(\lambda)}} \frac{\partial a_{m}}{\partial t_{k}}-\frac{k}{2} \sqrt{\lambda-a_{m}} \sigma_{k}(\lambda)\right)_{\lambda=a_{m}},
$$

which yields

$$
\frac{\partial a_{m}}{\partial t_{k}}=-k \frac{\widehat{\sigma}_{k}\left(a_{m}\right)}{\widehat{\psi}\left(a_{m}\right)} .
$$

This is the first equality in 8.105). To obtain the second identity in 8.105), it is sufficient to use (8.71) and to calculate the residue at the points $\left(a_{m}, 0\right), m=1,2,3,4$. The proof of 8.106) follows from (8.105) and from Rauch's variational formula [45]

$$
\frac{1}{4 \pi i} \frac{\partial B}{\partial a_{i}}=\operatorname{Res}_{\lambda=a_{i}} \frac{v(P) v(P)}{d \lambda^{2}}=\widehat{v}\left(a_{i}\right) \widehat{v}\left(a_{i}\right)
$$

In order to prove (8.107), we use 8.114) and 8.58), and this leads to

$$
\begin{aligned}
\frac{\partial}{\partial t_{k}} \widehat{\psi}\left(a_{m}\right) & =\frac{\partial}{\partial t_{k}}\left(\left.\frac{\pi i}{\left(\lambda-a_{m}\right)^{1 / 2}} \psi(\lambda)\right|_{\lambda=a_{m}}\right)=\left.\pi i\left(\frac{\partial}{\partial t_{k}} \frac{\psi(\lambda)}{\left(\lambda-a_{m}\right)^{1 / 2}}+\frac{\partial}{\partial \lambda} \frac{\psi(\lambda)}{\left(\lambda-a_{m}\right)^{1 / 2}} \frac{\partial a_{m}}{\partial t_{k}}\right)\right|_{\lambda=a_{m}} \\
& =\left.\left(\frac{k \sigma_{k}(\lambda)}{2\left(\lambda-a_{m}\right)^{1 / 2} d \lambda}+\frac{\mathcal{R}(\lambda)^{1 / 2}}{\left(\lambda-a_{m}\right)^{1 / 2}} \frac{\partial a_{m}}{\partial t_{k}}\left(h^{\prime}(\lambda)+\frac{1}{2} h(\lambda) \sum_{j} \frac{1}{\lambda-a_{j}}\right)\right)\right|_{\lambda=a_{m}} .
\end{aligned}
$$

Using (8.70) and (8.105), we obtain

$$
\begin{aligned}
\frac{\partial}{\partial t_{k}} \widehat{\psi}\left(a_{m}\right) & =-\frac{3}{2} k \widehat{\sigma}_{k}\left(a_{m}\right) \frac{h^{\prime}\left(a_{m}\right)}{h\left(a_{m}\right)}+\frac{k}{2} \frac{\left.\partial_{\lambda} P_{k}(\lambda)\right|_{\lambda=a_{m}}}{\sqrt{\prod_{j \neq m}\left(a_{m}-a_{j}\right)}}-k \frac{P_{k}\left(a_{m}\right)}{\sqrt{\prod_{j \neq m}\left(a_{m}-a_{j}\right)}} \sum_{j \neq m} \frac{1}{a_{m}-a_{j}} \\
& =-\left.\frac{3}{2} k \frac{\widehat{\sigma}_{k}\left(a_{m}\right)}{\widehat{\psi}\left(a_{m}\right)} \frac{\partial}{\partial \lambda}\left(h(\lambda) \sqrt{\prod_{j \neq m} \lambda-a_{j}}\right)\right|_{\lambda=a_{m}}+\left.\frac{k}{2}\left(\frac{\partial}{\partial \lambda} \frac{P_{k}(\lambda)}{\sqrt{\prod_{j \neq m}\left(\lambda-a_{j}\right)}}\right)\right|_{\lambda=a_{m}},
\end{aligned}
$$


which, by 8.58 and 8.75, implies 8.107).

\subsection{Proof of Theorem 2.11 and Theorem 2.12}

Define

$$
F_{1}=\frac{1}{24} \log \left(\frac{1}{2^{8}}\left(\frac{|\mathcal{A}|}{2 \pi}\right)^{12} \Delta(a)^{3} \prod_{j=1}^{4} \widehat{\psi}\left(a_{j}\right)\right),
$$

where $\Delta(a)=\prod_{1 \leq i<j \leq 4}\left(a_{j}-a_{i}\right)$ is the Vandermonde determinant of the branch points, $\mathcal{A}$ is the $\alpha$-period of the holomorphic differential defined in 8.36 , and $\widehat{\psi}$ has been defined in (8.58). This quantity appeared first in [1].

Combining Theorem 8.7 and Lemma 8.9 , we get, as $N \rightarrow \infty$,

$$
\begin{gathered}
-\frac{\partial}{\partial t_{k}} \log Z_{N}\left(V_{\vec{t}}\right)=N^{2} \frac{\partial F_{0}}{\partial t_{k}}-N(\log \theta(N \Omega ; B))^{\prime} \frac{\partial \Omega}{\partial t_{k}}+\frac{\partial F_{1}}{\partial t_{k}}-\frac{\partial}{\partial B} \log \theta(N \Omega ; B) \frac{\partial B}{\partial t_{k}} \\
+\left(\frac{F_{0}^{(3)}}{6}\left(\frac{(\theta(N \Omega ; B))^{\prime \prime \prime}}{\theta(N \Omega ; B)}\right)^{\prime}-(\log \theta(N \Omega ; B))^{\prime \prime} F_{1}^{(1)}\right) \frac{\partial \Omega}{\partial t_{k}}+\mathcal{O}\left(N^{-1}\right) .
\end{gathered}
$$

Consequently, we have

$$
\begin{aligned}
-\frac{\partial}{\partial t_{k}} \log Z_{N}\left(V_{\vec{t}}\right)=\frac{\partial}{\partial t_{k}}( & N^{2} F_{0}+F_{1}-\log \theta(N \Omega ; B)-\frac{\theta^{\prime}(N \Omega ; B)}{\theta(N \Omega ; B)} \frac{F_{1}^{(1)}}{N} \\
+ & \left.\frac{\theta^{\prime \prime \prime}(N \Omega)}{\theta(N \Omega ; B)} \frac{F_{0}^{(3)}}{6 N}+\mathcal{O}\left(N^{-1}\right)\right), \quad N \rightarrow \infty
\end{aligned}
$$

where $F_{0}$ and $F_{1}$ have been defined in 8.25 and 8.120 , and $F_{0}^{(3)}$ and $F_{1}^{(1)}$ have been defined in 8.102 and 8.101). From the above identity the proof of Theorem 2.11 follows easily.

Remark 8.10 The error $\mathcal{O}\left(N^{-1}\right)$ in 8.121 contains non-oscillatory terms of order $1 / N$ and smaller order terms. To determine such terms, it is necessary to calculate an extra term in the asymptotic expansion of the error matrix $R(z)$.

From Theorem 2.9, we can choose a continuous path $\vec{t}:[0,1] \rightarrow \mathbb{R}^{m}: s \mapsto \vec{t}(\tau)=$ $\left(t_{1}(\tau), \ldots, t_{m}(\tau)\right)$ in $\mathbb{R}^{m}$ such that $V_{\vec{t}(0)}(z)=z^{4}-4 z^{2}$, and $V_{\vec{t}(1)}(z)=V_{\vec{t}}(z)$ and for all $\tau \in[0,1], V_{\vec{t}(\tau)}$ is a two-cut regular potential. Therefore one can integrate $(2.24)$ to obtain

$$
\begin{aligned}
& \log Z_{N}\left(V_{\vec{t}}\right)-\log Z_{N}\left(V_{0}\right)=-N^{2}\left(F_{0}\left(V_{\vec{t}}\right)-F_{0}\left(V_{0}\right)\right)-F_{1}\left(V_{\vec{t}}\right)+F_{1}\left(V_{0}\right) \\
& \quad+\log \theta\left(N \Omega\left(V_{\vec{t}}\right) ; B\left(V_{\vec{t}}\right)\right)-\log \theta\left(N \Omega\left(V_{0}\right) ; B\left(V_{0}\right)\right)+\mathcal{O}(1 / N), \quad N \rightarrow \infty .
\end{aligned}
$$

By 2.10, this implies Theorem 2.12. 


\section{Acknowledgements}

TC was supported by the European Research Council under the European Union's Seventh Framework Programme (FP/2007/2013)/ ERC Grant Agreement n. 307074, by the Belgian Interuniversity Attraction Pole P07/18, and by F.R.S.-F.N.R.S.

TG was partially supported by PRIN Grant Geometric and analytic theory of Hamiltonian systems in finite and infinite dimensions of Italian Ministry of Universities and Researches and by the FP7 IRSES grant RIMMP Random and Integrable Models in Mathematical Physics.

KDT-RM was supported in part by the National Science Foundation under grants DMS-0800979 and DMS-1401268 and by by the FP7 IRSES grant RIMMP Random and Integrable Models in Mathematical Physics.

\section{References}

[1] G. Akemann, Higher genus correlators for the hermitian matrix model with multiple cuts, Nucl. Phys. B 482 (1996), 403-430.

[2] G. Akemann, D. Dalmazi, P.H. Damgaard, and J.J.M. Verbaarschot, QCD3 and the replica method, Nucl. Phys. B 601 (2001), 77-124.

[3] G.W. Anderson, A. Guionnet, and O. Zeitouni, "An introduction to random matrices", Cambridge Studies in Advanced Mathematics 118, Cambridge University Press, Cambridge, 2010.

[4] M. Bertola, The dependence on the monodromy data of the isomonodromic tau function, Comm. Math. Phys. 294 (2010), no. 2, 539-579.

[5] M. Bertola, B. Eynard, and J. Harnad, Partition functions for matrix models and isomonodromic tau functions, J. Phys. A 36 (2003), 3067-3983.

[6] M. Bertola, B. Eynard, and J. Harnad, Semiclassical orthogonal polynomials, matrix models and isomonodromic tau functions, Comm. Math. Phys. 263 (2006), no. 2, 401-437.

[7] D. Bessis, C. Itzykson, and J.-B. Zuber, Quantum field theory techniques in graphical enumeration, Adv. Appl. Math. 1 (1980), 109-157.

[8] P. Bleher and A. Its, Asymptotics of the partition function of a random matrix model, Ann. Inst. Fourier 55 (2005), no. 6, 1943-2000.

[9] P. Bleher and A. Its, Double scaling limit in the random matrix model: the Riemann-Hilbert approach, Comm. Pure Appl. Math. 56 (2003), 433-516.

[10] G. Bonnet, F. David, and B. Eynard, Breakdown of universality in multi-cut matrix models, J. Phys. A 33 (2000), 6739-6768.

[11] G. Borot and A. Guionnet, Asymptotic expansion of beta matrix models in the multi-cut regime, arXiv:1303.1045.

[12] A. Boutet de Monvel, L. Pastur, and M. Shcherbina, On the statistical mechanics approach in the random matrix theory. Integrated density of states, J. Stat. Phys. 79 (1995), 585-611. 
[13] M.J. Bowick and E. Brézin, Universal scaling of the tail of the density of eigenvalues in random matrix models, Phys. Lett. B 268 (1991), 21-28.

[14] E. Brézin, C. Itzykson, G. Parisi, and J.-B. Zuber, Planar diagrams, Comm. Math. Phys. 59 (1978), 35-51.

[15] E. Brézin, E. Marinari, and G. Parisi, A non-perturbative ambiguity free solution of a string model, Phys. Lett. B 242 (1990), no. 1, 35-38.

[16] L.O. Chekhov and B. Eynard, Matrix eigenvalue model: Feynman graph technique for all genera, JHEP 026 (2006).

[17] T. Claeys and A. Kuijlaars, Universality in unitary random matrix ensembles when the soft edge meets the hard edge, in "Integrable Systems and Random Matrices: in honor of Percy Deift", Contemporary Mathematics 458, Amer. Math. Soc., Providence R.I. 2008, 265-280.

[18] T. Claeys and M. Vanlessen, Universality of a double scaling limit near singular edge points in random matrix models, Comm. Math. Phys. 273 (2007), 499-532.

[19] P. Deift, A. Its, A. Kapaev, and X. Zhou, On the algebro-geometric integration of the Schlesinger equations, Comm. Math. Phys. 203 (1999), no. 3, 613-633.

[20] P. Deift, T. Kriecherbauer, and K.T-R McLaughlin, New results on the equilibrium measure for logarithmic potentials in the presence of an external field, J. Approx. Theory 95 (1998), 388-475.

[21] P. Deift, T. Kriecherbauer, K.T-R McLaughlin, S. Venakides, and X. Zhou, Uniform asymptotics for polynomials orthogonal with respect to varying exponential weights and applications to universality questions in random matrix theory, Comm. Pure Appl. Math. 52 (1999), 1335-1425.

[22] P. Deift, T. Kriecherbauer, K.T-R McLaughlin, S. Venakides, and X. Zhou, Strong asymptotics of orthogonal polynomials with respect to exponential weights, Comm. Pure Appl. Math. 52 (1999), 1491-1552.

[23] P. Di Francesco, P. Ginsparg, and J. Zinn-Justin, 2D Gravity and Random Matrices, Phys. Rep. 254 (1995), 1-133.

[24] B. Dubrovin and Y. Zhang, Bi-Hamiltonian hierarchies in 2D topological field theory at one-loop approximation, Comm. Math. Phys. 198 (1998), no. 2, 311361.

[25] N.M. Ercolani and K. T-R McLaughlin, Asymptotics of the partition function for random matrices via Riemann-Hilbert techniques and applications to graphical enumeration, Int. Math. Res. Not. 2003 (2003), no. 14, 755-820.

[26] B. Eynard, Large N expansion of convergent matrix integrals, holomorphic anomalies, and background independence, JHEP 0903 (2009), 003, 20 pp.

[27] B. Eynard and N. Orantin, Invariants of algebraic curves and topological expansion, Commun. Number Theory Phys. 1 (2007), no. 2, 347-452.

[28] J. Fay, "Theta-functions on Riemann surfaces", Lecture Notes in Math. 352, Springer, 1973. 
[29] A.S. Fokas, A.R. Its, and A.V. Kitaev, The isomonodromy approach to matrix models in 2D quantum gravity, Comm. Math. Phys. 147 (1992), 395-430.

[30] P.J. Forrester, "Log-gases and random matrices", London Mathematical Society Monographs Series 34, Princeton University Press, Princeton, NJ, 2010.

[31] T. Grava, Partition function for multi-cut matrix models, J. Phys. A 39 (2006), no. $28,8905-8919$.

[32] T. Grava and F.-R. Tian, Large parameter behavior of equilibrium measures, Commun. Math. Sci. 4 (2006), no. 3, 551-573.

[33] M. Jimbo, T. Miwa, and K. Ueno, Monodromy preserving deformation of linear ordinary differential equations with rational coefficients I, Physica D (1981), 306352 .

[34] K. Johansson, On fluctuations of eigenvalues of random Hermitian matrices, Duke Math. J. 91 (1998), 151-204.

[35] J. Jurkiewicz, Regularisation of one-matrix models, Phys. Lett.B 245 (1990), $178-184$.

[36] A. Kitaev and D. Korotkin, On solutions of the Schlesinger equations in terms of Theta-functions, Int. Math. Res. Notices, 17 (1998), 877-905.

[37] A. Kokotov and D. Korotkin, Tau-functions on Hurwitz spaces. Math. Phys. Anal. Geom. 7, (2004), no. 1, 4796.

[38] D. Korotkin, Solution of matrix Riemann-Hilbert problems with quasi-permutation monodromy matrices, Math. Ann. 329 (2004), 335-364.

[39] I. Krasovsky, Correlations of the characteristic polynomials in the Gaussian Unitary Ensemble or a singular Hankel determinant, Duke Math. Journal 139 (2007), no. $3,581-619$.

[40] I.M. Krichever, Spectral theory of two-dimensional periodic operators and its applications (Russian), Uspekhi Mat. Nauk 44 (1989), no. 2 (266), 121-184; translation in Russian Math. Surveys 44 (1989), no. 2, 145-225.

[41] A.B.J. Kuijlaars and K.T-R McLaughlin, Generic behavior of the density of states in random matrix theory and equilibrium problems in the presence of real analytic external fields, Comm. Pure Appl. Math. 53 (2000), 736-785.

[42] T. Leblé and S. Serfaty, Large deviation principle for empirical fields of Log and Riesz gases, arXiv:1502.02970.

[43] A. Martinez-Finkelshtein, R. Orive, and E.A. Rakhmanov, Phase transitions and equilibrium measures in random matrix models, arXiv:1302.3647.

[44] F. Mezzadri and M.Y. Mo, On an average over the Gaussian Unitary Ensemble, Int. Math. Res. Notices 2009 (2009), article ID rnp062, 30 pages.

[45] H.E. Rauch, Weierstrass points, branch points, and moduli of Riemann surfaces, Comm. Pure Appl. Math. 12 (1959), 543-560. 
[46] E.B. Saff and V. Totik, "Logarithmic Potentials with External Fields", SpringerVerlag, New-York (1997).

[47] E. Sandier and S. Serfaty, 1D log gases and the renormalized energy: crystallization at vanishing temperature, arXiv:1303.2968.

[48] R. Schiappa and R. Vaz, The resurgence of instantons: multi-cuts Stokes phases and the Painlevé II equation, arXiv:1302.5138.

[49] M. Shcherbina, Fluctuations of linear eigenvalue statistics of $\beta$ matrix models in the multi-cut regime, arxiv:1205:7062. 